\title{
Sequential Designs for Individualized Dosing in Phase I Cancer Clinical Trials
}

\author{
Xuezhou Mao
}

\author{
Submitted in partial fulfillment of the \\ requirements for the degree of \\ Doctor of Philosophy \\ under the Executive Committee \\ of the Graduate School of Arts and Sciences
}

COLUMBIA UNIVERSITY

2014 
(c) 2014

Xuezhou Mao

All rights reserved 


\begin{abstract}
Sequential Designs for Individualized Dosing in Phase I Cancer Clinical Trials

Xuezhou Mao
\end{abstract}

This dissertation presents novel sequential dose-finding designs that adjust for inter-individual pharmacokinetic variability in phase I cancer clinical trials. Unlike most traditional dosefinding designs whose primary goals are the determination of a maximum safe dose, the goal of our proposed designs is to estimate a patient-specific dosing function such that the responses of patients can achieve a target safety level.

Extending from a single compartment model in the pharmacokinetic theory, we first postulate a linear model to describe the relationship between the area under concentrationtime curve, dose and predicted clearance. We propose a repeated least squares procedure that aims to sequentially determine dose according to individual ability of metabolizing the drug. To guarantee consistent estimation of the individualized dosing function at the end of a trial, we apply repeated least squares subject to a consistency constraint based on an eigenvalue theory for stochastic linear regression. We empirically determine the convergence rate of the eigenvalue constraint using a real data set from an irinotecan study in colorectal carcinoma patients, and calibrate the procedure to minimize a loss function that accounts for the dosing costs of study subjects and future patients. When compared to the traditional body surface area and an equation based dosing methods, the simulation results demonstrate that the repeated least squares procedure control the dosing cost and allow for precise estimation of the dosing function.

Furthermore, in order to enhance the generality and robustness of the dose-finding designs, we generalize the linear association to a nonlinear relationship between the response 
and a linear combination of dose and predicted clearance. We propose a two-stage sequential design, the semiparametric link-adapted recursion, which targets at individualizing dose assignments meanwhile adapting for an unknown nonlinear link function connecting the response and dose along with predicted clearance. The repeat least squares with eigenvalue constraint design is utilized as the first stage, and the second stage recursively applies an iterative semiparametric least squares approach to estimate the dosing function and determine dosage for next patient. The simulation results demonstrate that: at first, the performance of repeated least squares with eigenvalue constraint design is acceptably robust to model misspecifications; at second, as its performance is close to that of repeated least squares procedure under parametric models, the semiparametric link-adapted recursion does not sacrifice much estimation accuracy to gain robustness against model misspecifications; at last, compared to the repeated least squares procedure, the semiparametric link-adapted recursion can significantly improve the dosing costs and estimation precision under the semiparametric models. 


\section{Contents}

List of Tables $\quad$ iv

List of Figures $\quad$ v

1 Introduction $\quad 1$

1.1 Clinical Background . . . . . . . . . . . . . . . . . 1

1.1.1 Phase I Cancer Clinical Trials . . . . . . . . . . . . . . . . 1

1.1.2 Pharmacokinetics of Phase I Trials . . . . . . . . . . . . . . 2

1.2 Review of Dose-Finding Designs for Phase I Cancer Trials . . . . . . . . . . 4

1.2.1 The $3+3$ Algorithm . . . . . . . . . . . . . . . . . 4

1.2.2 Continual Reassessment Method . . . . . . . . . . . . . . . 5

1.2.3 Escalation with Overdose Control . . . . . . . . . . . . . 7

1.2.4 Stochastic Approximation . . . . . . . . . . . . . . . 7

1.3 Review of Individualized Dose-Finding Designs for Phase I Cancer Trials . . 9

1.3.1 Two-Sample Continual Reassessment Methods . . . . . . . . . . . . . 10

1.3.2 Nonparametric Dose-Finding Design with Ordered Groups . . . . . . 11

1.3.3 Individualized Continual Reassessment Method . . . . . . . . . . . . 13

1.3.4 Individualized Escalation with Overdose Control . . . . . . . . . . 15

1.4 Motivations . . . . . . . . . . . . . . . . . . . . . . 18

1.4.1 Literature Review Discussions . . . . . . . . . . . . . . . 18 
1.4.2 Motivating Trial: the Irinotecan Study . . . . . . . . . . . . . . . 20

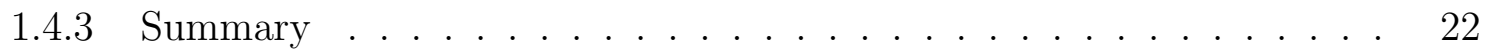

2 Repeated Least Squares Procedure $\quad 24$

2.1 Linear Dose-Response Model . . . . . . . . . . . . . . . . . . . 24

2.2 Unconstrained Repeated Least Squares Design . . . . . . . . . . . . . . . 26

2.3 Repeated Least Squares with Eigenvalue Constraint Design . . . . . . . . . . 28

2.4 Coherence Restriction . . . . . . . . . . . . . . . . . 30

2.5 The Eigenvalue Theorem and An Example . . . . . . . . . . . . . . 32

3 Applications of Repeated Least Squares Procedure 37

3.1 Loss Criteria . . . . . . . . . . . . . . . . . . . 37

3.2 Calibration Scenarios Generation . . . . . . . . . . . . . . 39

3.3 Calibrating the Eigenvalue Constraint . . . . . . . . . . . . . . . . 42

3.4 An Illustrative Trial . . . . . . . . . . . . . . . . . . . . . . . 48

3.5 Design Comparison under Linear Models . . . . . . . . . . . . . . . . 51

4 Semiparametric Link-Adapted Recursion $\quad 58$

4.1 Semiparametric Dose-Response Model . . . . . . . . . . . . . . . . . . . . 58

4.2 Isotonic Semiparametric Least Squares Estimation . . . . . . . . . . . . . 60

4.3 Semiparametric Link-Adapted Recursion Design . . . . . . . . . . . . . 65

4.4 Applications of Semiparametric Link-Adapted Recursion . . . . . . . . . . . 71

4.4.1 Fitting Semiparametric Models to Irinotecan Data . . . . . . . . . . . 71

4.4 .2 An Illustrative Trial . . . . . . . . . . . . . . 76

4.4.3 Design Comparison under Semiparametric Models . . . . . . . . . . 78

4.5 Theoretical Results ...................... 86

5 Conclusions and Discussions $\quad 99$ 
Bibliography 


\section{List of Tables}

1.1 The Traditional $3+3$ Algorithm . . . . . . . . . . . . . . . 5

1.2 Summary of the Irinotecan Data . . . . . . . . . . . . . . . . . . . . . 22

3.1 True Scenarios in Design Comparison under Linear Model . . . . . . . . . . 51

3.2 Summary of Design Comparison under Linear Model . . . . . . . . . . . . 53

4.1 Two-Stage SLAR under Linear Models . . . . . . . . . . . . . . . . 80

4.2 Two-Stage SLAR under Nonlinear Models . . . . . . . . . . . . . 83 


\section{List of Figures}

2.1 Fitting a Linear Model to the Irinotecan Data . . . . . . . . . . . . . . 25

3.1 Trade-off between Dosing Cost and MISE as $\left(\delta_{1}, \delta_{2}\right)$ grows $\ldots \ldots \ldots 44$

3.2 The Impacts of $\delta_{1}$ and $\delta_{2}$ on Dosing Cost and MISE . . . . . . . . . . 46

3.3 The Impacts of $\delta_{1}$ and $\delta_{2}$ on Loss Function . . . . . . . . . . . . 47

3.4 An Illustrative Trial . . . . . . . . . . . . . . . . . . . . . . . . . . . . 49

3.5 True Dosing Functions of Design Comparison under Linear Model . . . . . 52

3.6 Comparison of Average Dosing Cost per Each Subject . . . . . . . . . . . 56

4.1 Profile of Residual Square Sums from Kernel Smoothing _ . . . . . . . . 72

4.2 Fitting a Nonlinear Link to Irinotecan Data . . . . . . . . . . . . . 74

4.3 Nonlinear Links used in Simulations . . . . . . . . . . . . . . . . . 75

4.4 An Illustrative Trial using SLAR . . . . . . . . . . . . . . . . 77

4.5 Average Dosing Cost per Each Subject using SLAR . . . . . . . . . . . . 85 


\section{Dedication}

To my mother, my farther, and my wife Mei, who always stand by me, support me, and encourage me throughout this pursuing journey. 


\section{Acknowledgments}

I would like to express my deepest appreciation to my advisor, Dr. Ying Kuen Cheung, who provides me with mentoring, support and discipline throughout this endeavor. I sincerely appreciate that, Dr. Cheung leads me into the world of phase I dose-finding designs, encourages me to explore the unknowns, helps me overcome difficulties, and teaches me the ways of scientific research. It is a pleasant, invaluable and unforgettable experience to learn from and work with Dr. Cheung, which will benefit me in the rest of my life.

I particularly thank Dr. Wei-Yann Tsai, Dr. Yuanjia Wang, Dr. Quixuan Chen, and Dr. Kevin Kalinsky for their precious time on serving as the examining committee members and reviewing my dissertation manuscript. It is their valuable suggestions and comments that helped me improve the dissertation and inspired my further explorations beyond this work in the future.

I also want to express special thank to Dr. Hui Quan from Sanofi-Aventis U.S. Inc., who offered me with an invaluable intern opportunity to enrich my knowledge and research experiences in clinical trials, and led me to learn the applications of statistical methods in pharmaceutical industry. I would like to thank Dr. Zhezhen Jin, Dr. Ying Wei, Dr. Bin Cheng, and Dr. Shing Lee, who also kindly provided me with great help in my learning and study experience at Columbia University. Moreover, special thanks are also given to Chih-Chi Hu, Hsin-Wen Chang, Nanshi Sha, Huaihou Chen, Jimmy K. Duong, and Justine Herrera, who helped me much with my study and life.

Thank you very much for all the people who have kindly given me helps and made this thesis possible. It is impossible to especially remember all of them one-by-one here, and I sincerely apologize to those who I inadvertently leave out. 


\section{Chapter 1}

\section{Introduction}

\subsection{Clinical Background}

\subsubsection{Phase I Cancer Clinical Trials}

A phase I clinical trial is the earliest stage in clinical drug development, and is typically carried out in a small number of subjects. The goal of phase I trials is to investigate toxicity and pharmacokinetics $(\mathrm{PK})$ of a new drug, in order to set the basis for later investigation of efficacy. Since the phase I trial is the first step where subjects are treated experimentally with a new drug, the safety and feasibility of the new treatment have the top priority among its concerns.

For non-life-threatening diseases, phase I trials are usually conducted on healthy volunteers, as long as the expected toxicity is mild and can be controlled without harm. For life-threatening diseases such as cancer and AIDS, phase I trials are conducted with patients because of the aggressiveness and possible harmfulness of drug-related cytotoxicity, possible systemic treatment effects, and the high interest in the new drug's efficacy in those patients directly. The instruction of methodology throughout this dissertation will be focused on the phase I cancer trials. 
Traditional phase I trials of a novel anticancer drug aim to determine a maximum safe dose along with standardized treatment schedule for further investigation in later phases. The dose is the so-called maximum tolerated dose (MTD), which is a maximum test dose that does not exceed an acceptable level of toxicity. Most dose-finding designs for phase I cancer trials assume that there exists a monotone dose-toxicity and dose-tumor response relationship, such that the higher the dose level is, the drug is not only more effective but also more toxic.

In the context of traditional phase I trials, the primary endpoint $Y$ is usually a binary indicator of occurrence of the dose limiting toxicity (DLT), with a pre-specified target toxicity rate $\theta \in(0,1)$; and, the dosage $X$ usually takes values from a pre-selected set of discrete dose levels denoted by $\mathcal{D}=\left\{d_{k}, k=1, \cdots, K\right\}$ with $d_{k}<d_{k+1}$. In the rest of Chapter 1 , if without special indication, the aforementioned notations will be used continuously.

Definition 1 (MTD-surrogacy perspective) MTD from a surrogate-for-efficacy perspective, denoted by $\nu$, is defined as the dose level with toxicity probability closest to the target probability $\theta$, that is $\nu=\arg \min _{d_{k} \in \mathcal{D}}\left|p_{k}-\theta\right|$, where $p_{k}=P\left(Y=1 \mid X=d_{k}\right)$.

According to the surrogacy perspectives definitions, the major statistical issue in the dose finding of traditional phase I cancer trials, is the estimation of MTD, which can be identified as a percentile estimation problem. Several commonly used dose-finding designs will be briefly summarized in Section 1.2.

\subsubsection{Pharmacokinetics of Phase I Trials}

An important but often neglected secondary objective of a phase I trial is the Pharmacokinetics, which investigates the absorption, distribution, metabolism and excretion of a drug in human body. All drugs share a common feature that they are formulated to have effects on some body system. In oncology, the effects include shrinking the size of a tumor, reducing the tumor growth rate, and protecting non-cancer cell from potentially harmful effects 
of chemotherapy. Pharmacokinetics studies what happens to drugs once they enter human body, which often will entail drawing blood samples to measure the concentration of the compound, possibly along with metabolites, over time. Important PK parameters include the plasma concentration, area under the concentration-time curve (AUC), and clearance, where AUC measures a subject's overall exposure to a drug and clearance quantifies a subject's ability of eliminating a drug. The PK measurements can be either incorporated into the dose-response model as a covariate or utilized to define the primary endpoint.

The relationship between drug concentration and time is often characterized by a system of differential equations, which is the so-called compartment model. These differential equations describe the instantaneous change in drug concentration or drug metabolites within each compartment, with direct or indirect communication among compartments. The compartments in the model are based on the notion that, the drug circulates through human body in the blood stream and may visit other parts of the body before it is eliminated. The plasma or blood compartment may be considered as the central compartment for a drug that is infused intravenously though, the drug will likely pass through the liver, kidneys, the tumor hopefully, and then returns to the plasma from which it is eliminated at last. The transit between plasma and the other organs forms part of the system of differential equations characterizing the change in concentration over time.

Most anticancer drugs share a common feature that their therapeutic dosage windows are narrow. In the past decades, the rapidly growing understanding of the pharmacokinetics of anti-cancer drugs via the PK models enable us with exactly distinguishing the abilities of eliminating drug metabolites among different patients. In the presence of large interindividual PK variability, if all patients are still treated at a common dose, they could be either overdosed or underdosed. Both would cause sever ethical issues, as the former would lead to unacceptable toxicities in some patients and the latter would result in insufficient efficacy for some others. 
Therefore, if the potential inter-individual PK variation could be taken into consideration when determining doses for patients, then a patient-specific maximal tolerated dose could be recommended to ideally reduce ethical issues along with the traditional "one-size-fit-all" dosing concept. This is one major motivation of the development of individualized dosing methods for phase I cancer clinical trials.

\subsection{Review of Dose-Finding Designs for Phase I Can- cer Trials}

The dose-finding designs for traditional phase I cancer trials usually allocate MTD within a set of discrete dose levels $\mathcal{D}=\left\{d_{k}, k=1, \cdots, K\right\}$, which is specified in the planning stage of the study. Typically in a sequential manner, a trial begins with a small number of patients been treated at a starting dose, and decision to escalate or not depends on a dosing algorithm that adapts to the occurrence of toxicity events. When a dose level is reached with unacceptable toxicity, then the trial is stopped. In this section, the basics of frequently used dose-finding designs for phase I cancer trials will be introduced. A comprehensive and in-depth review of can be found in Cheung [2011].

\subsubsection{The 3+3 Algorithm}

The method (see Table 1.2.1) starts a trial at a low dose and escalates after every 3 to 6 patients per dose; the recommended dose is defined as the largest dose with fewer than 2 patients experiencing a predefined dose limiting toxicity (DLT) during the first course of treatment.

At present, the $3+3$ algorithm is still the most widely used design in practices, mainly because of its simplicity and feasibility. Since the dose escalation rules can be tabulated before a trial starts, the clinical investigators can make dose decisions during a trial without 
the help from a statistician.

Table 1.1: The Traditional 3+3 Algorithm

\begin{tabular}{ccl}
\hline $\mathrm{n}^{1}$ & Number of DLT & \multicolumn{1}{c}{ Action } \\
\hline 3 & 0 & Escalate to the next higher dose $d_{i+1}$ \\
3 & 1 & Treat 3 additional patients at the current dose $d_{i}$ \\
6 & 1 & $\begin{array}{l}\text { Escalate to the next higher dose } d_{i+1} \\
3 \text { or } 6\end{array}$ \\
& & $\begin{array}{l}\text { Stop escalation and terminate the trial } \\
\text { The MTD is estimated by the dose immediately below } \\
\text { the terminating dose }\end{array}$ \\
\hline
\end{tabular}

However, the $3+3$ algorithm has many limitations. For instance, it tends to treat many patients at a low and inefficacious dose, due to the conservative escalation scheme, and thus has significant ethical issues. The $3+3$ algorithm has no statistical justification. There is no intrinsic property in the method to stop escalation at any given percentile, and hence the distribution of MTD depends arbitrarily on the underlying dose-toxicity curve and the number of test doses. Moreover, it is obvious that the $3+3$ algorithm overlooks any possible between-subject heterogeneity.

\subsubsection{Continual Reassessment Method}

The continual reassessment method (CRM) [O'Quigley et al., 1990] is the first model-based design in dose-finding methodologies for phase I cancer clinical trials. Depending on the estimation methods, there are generally two types of CRM designs: the one-stage Bayesian CRM and the two-stage maximum likelihood CRM. In this section, we briefly introduce the one-stage Bayesian CRM as an example, and more systematic reviews can be found in Cheung [2011].

The toxicity probability of the binary toxicity response $\pi(x)=P\left\{y_{i}=1 \mid x\right\}$ is assumed a monotone increasing function in dosage $x$. The trial objective is to determine the dose level that is associated with a target toxicity rate $\theta$. CRM postulates the true dose-toxicity curve

\footnotetext{
${ }^{1} \mathrm{n}$ is the cumulative sample size.
} 
$\pi(x)$ to be $F\left(x, \beta_{0}\right)$ for some true parameter value $\beta_{0}$, and requires $F(x, \beta)$ to be strictly increasing in the dose $x$.

One of the most commonly used link functions in CRM is the one-parameter logistic function

$$
F(x, \beta)=\frac{\exp \left(a_{0}+\beta x\right)}{1+\exp \left(a_{0}+\beta x\right)},
$$

where the intercept $a_{0}$ is a fixed constant, and the parameter $\beta$ is restricted to be positive in order to ensure an increasing dose-toxicity relationship.

CRM launches a trial by treating the first patient at a pre-determined starting dose $x_{1}$, which is believed to have the closest toxicity probability to the target rate $\theta$ based on existing knowledge. Each subsequent $x_{i}$ is determined sequentially based on all the previous observations $\left\{\left(x_{j}, y_{j}\right): 1 \leq j<i\right\}$, such that

$$
x_{i}=\arg \min _{d_{k} \in \mathcal{D}}\left|F\left(d_{k}, \hat{\beta}_{i-1}\right)-\theta\right|
$$

where $\hat{\beta}_{i-1}=\frac{\int_{0}^{\infty} \beta L_{i-1}(\beta) \phi(\beta) d \beta}{\int_{0}^{\infty} L_{i-1}(\beta) \phi(\beta) d \beta}$ is the posterior mean of $\beta$ given the current data, $\beta$ is assumed to follow a normal prior distribution, and the binomial likelihood is as follows

$$
L_{i-1}(\beta)=\prod_{j=1}^{i-1}\left\{F\left(x_{j}, \beta\right)^{y_{j}}\left[1-F\left(x_{j}, \beta\right)\right]^{1-y_{j}}\right\} .
$$

The sequential dose allocation procedure is continued until a prespecified sample size $n$ is reached, and the estimated MTD recommended by this CRM trial is $x_{n+1}$.

In words, CRM attempts to treat each patient at the current best estimate of MTD, that is a dose whose corresponding estimated toxicity probability is the closest to the target rate $\theta$ among all dose levels. 


\subsubsection{Escalation with Overdose Control}

The escalation with overdose control (EWOC) design takes the notions of continual reassessment method, but estimates MTD with respect to an asymmetric loss function that places larger penalty on overdosing than underdosing.

For a given $\alpha \in(0,1)$, the loss function is defined as follows

$$
L_{\alpha}(x, \nu)= \begin{cases}\alpha(\nu-x), & \text { if } x \leq \nu, \text { that is, if } x \text { is an underdose } \\ (1-\alpha)(x-\nu), & \text { if } x>\nu, \text { that is, if } x \text { is an overdose }\end{cases}
$$

where $\nu$ is true MTD and $\alpha \leq 0.5$ usually. The loss incurred by treating a patient at $\delta$ units above the MTD is $\frac{1-\alpha}{\alpha}$ times greater than the loss associated with treating the patient at $\delta$ units below MTD. For each subject, EWOC minimizes the Bayesian risk with respect to the above loss function

$$
x_{i+1}=\arg \min _{x \in \mathcal{D}} E_{i}\left(L_{\alpha}(x, \nu)\right),
$$

where $E_{i}($.$) is the expectation with respect to the posterior distribution of MTD \nu$ given the current observations.

\subsubsection{Stochastic Approximation}

A nonparametric dose-response model is considered as follows:

$$
Y_{i}=M\left(X_{i}\right)+\epsilon_{i}
$$

where the regression function $M(x)=E(Y \mid X=x)$ is continuously differentiable in a neighborhood of $x_{0}$, denoted as $B\left(x_{0}\right)$, and has a unique root $x_{0}$ at a target mean level $\theta$ such that $M\left(x_{0}\right)=\theta$ with $M^{\prime}\left(x_{0}\right)=\beta>0$, and the random errors $\left\{\epsilon_{i}\right\}$ are i.i.d. with zero mean and finite variance $\sigma_{0}^{2}$. In the special case when $Y$ is a binary toxicity response and $X$ is 
the dose level, the regression function is equal to the toxicity probability at a dose, that is, $M(x)=\pi(x)=P(Y=1 \mid x)$.

Robbins and Monro [1951] introduce the first stochastic approximation method to address the problem of sequentially approaching the root to $M(x)=\theta$. The procedure starts the trial at a pre-specified dose level $x_{1}=d_{1}$, and approaches $x_{0}$ with successive approximation $x_{i}$ for $i>1$ recursively. The main idea of the Robbins-Monro procedure is to approximate the regression function in $B\left(x_{0}\right)$ by the first order Taylor expansion:

$$
M(x) \approx M\left(x_{0}\right)+M^{\prime}\left(x_{0}\right)\left(x-x_{0}\right)=\theta+\beta\left(x-x_{0}\right), \quad \forall x \in B\left(x_{0}\right),
$$

Then, based on the approximation $y_{i}=\theta+\beta\left(x_{i}-x_{0}\right)$, there is $x_{0}=x_{i}-\frac{1}{\beta}\left(y_{i}-\theta\right)$, which leads to the following recursion

$$
x_{i+1}=x_{i}-\frac{1}{i b}\left(y_{i}-\theta\right) \text {, }
$$

for some constant $b>0$ that is chosen by investigators.

The Robbins-Monro procedure is a nonparametric method. Under mild assumptions about the regression function $M(x)$ and the response $Y$, it can yield a consistent sequence such that $x_{i} \stackrel{a . s .}{\longrightarrow} x_{0}$ as $i \rightarrow \infty$. If in addition the constant $b$ is chosen so that $b<2 M^{\prime}\left(x_{0}\right)$,

$$
\sqrt{i}\left(x_{i}-x_{0}\right) \stackrel{d}{\rightarrow} N\left(0, \frac{\sigma_{0}^{2}}{b(2 \beta-b)}\right)
$$

Lai and Robbins [1979] modify the Robbins-Monro procedure via replacing $b$ with a consistent estimator of $\beta$, which is $\hat{b}_{n}$ obtained by recursively applying the least squares fit to the current observations $\left\{\left(x_{1}, y_{1}\right), \cdots,\left(x_{n}, y_{n}\right)\right\}$.

$$
x_{i+1}=x_{i}-\frac{1}{i \hat{b}_{i}}\left(y_{i}-\theta\right)
$$


where $\hat{b}_{i}=\max \left\{\underline{b}, \min \left(\hat{\beta}_{i}, \bar{b}\right)\right\}$ with pre-selected lower and upper bounds $\underline{b}$ and $\bar{b}$. Lai and Robbins [1979] also show that $\left\{x_{i}\right\}$ is consistent for $x_{0}$ and $\sqrt{i}\left(x_{i}-x_{0}\right) \stackrel{d}{\rightarrow} N\left(0, \frac{\sigma_{0}^{2}}{\beta^{2}}\right)$.

\subsection{Review of Individualized Dose-Finding Designs for Phase I Cancer Trials}

By suggesting a common MTD to all subjects, the traditional dosing concept does not allow for potential between-individual differences in susceptibility to a drug. Moreover, MTD is commonly defined with respect to clinical toxicity, however, it becomes increasingly important to consider biomarker expression or PK endpoints, with the use of molecular targeted therapies that demonstrate large PK variability.

Recent improvements in understanding drug metabolism have triggered the development of novel dose-finding designs that can accommodate inter-patient variation in drug tolerance. The basic idea of individualized dosing in early phase cancer trials is to find a dosing algorithm based on baseline characteristics that are believed to alter susceptibility to a drug. Instead of a constant MTD, an individualized trial aims at achieving a patient-specific dosing function, which depends on prognostic covariates and corresponds to a target response level.

For example, O'Quigley et al. [1999], O'Quigley and Paoletti [2003] and Ivanova and Wang [2006] propose designs that determine dosage adjusted for prognostic group-level distinction. Also, Piantadosi and Liu [1996] and Babb and Rogatko [2001] develop designs that individualize dose assignments while adjusting for measurable patient-specific characteristics, such as the PK measurements or ancillary pre-treatment information, in order to attain a target drug exposure. Potential benefits of these individualized dosing approaches include: the improvements in both safety and efficacy of the new treatment, enhancing the accuracy of dose escalation and dose-response model estimation, as well as the flexibility of dose recommendation in terms of allowing for adjustment with individual patient needs. 


\subsubsection{Two-Sample Continual Reassessment Methods}

In phase I cancer trials, it is common that patients are categorized into two or more subgroups, for example, according to either age categories or some previous treatment status, which is known to be related to individual tolerability to the new treatment. O'Quigley et al. [1999] extend the continual reassessment method to determine the target dose levels for two potentially heterogeneous patient subgroups, which is the so-called two-sample CRM.

The notions regarding the response and dosage all remains the same as those of CRM. The target toxicity rates for the two subgroups are assumed to be the same, that is $\theta \in(0,1)$. The set of dose levels $\mathcal{D}=\left\{d_{k}, k=1, \cdots, K\right\}$ are also assumed to be the same for the two subgroups. An extra covariate $G$ is introduced to as a subgroup indicator.

The true dose-response curves for the two subgroups are modeled as follows:

$$
\begin{aligned}
& \pi_{1}(x)=P(Y=1 \mid X=x, G=1)=\psi_{1}(x, \beta), \\
& \pi_{2}(x)=P(Y=1 \mid X=x, G=2)=\psi_{2}(x, \beta, b),
\end{aligned}
$$

where $(\beta, b)$ is a pair of parameters. The parameterization reflects that the two groups share a common $\beta$, while distinguish from each other in the parameter $b$. The functions $\psi_{1}$ and $\psi_{2}$ should be selected to ensure the existence of the solutions to $\psi_{1}(x, \beta)=\theta$ and $\psi_{2}(x, \beta, b)=\theta$ respectively, for example a commonly choice is the following logistic function:

$$
\left\{\begin{array}{l}
\psi_{1}(x, \beta)=\frac{\exp \left(a_{0}+\beta x\right)}{1+\exp \left(a_{0}+\beta x\right)}, \quad \beta>0 \text { and } a_{0} \text { is some known constant } \\
\psi_{2}(x, \beta, b)=\frac{b \exp \left(a_{0}+\beta x\right)}{1+b \exp \left(a_{0}+\beta x\right)}, \quad \beta>0, b>0 \text { and } a_{0} \text { is some known constant }
\end{array}\right.
$$

where the second curve represents a shift from the first one. 
Given the observations $\left(y_{i}, x_{i}, G_{i}\right), i=1, \cdots, k$, the joint likelihood is as follows:

$L_{k}(\beta, b)=\prod_{i=1}^{k}\left[\psi_{1}\left(x_{i}, \beta\right)^{y_{i}}\left(1-\psi_{1}\left(x_{i}, \beta\right)\right)^{1-y_{i}}\right]^{1_{\left(G_{i}=1\right)}}\left[\psi_{2}\left(x_{i}, \beta, b\right)^{y_{i}}\left(1-\psi_{2}\left(x_{i}, \beta, b\right)\right)^{1-y_{i}}\right]^{1_{\left(G_{i}=2\right)}}$.

whose maximizers are denoted as $\left(\hat{\beta}_{k}, \hat{b}_{k}\right)$. Both the Bayesian methods and maximum likelihood methods could be used to estimate the parameters.

If the $(k+1)$ th patient belongs to subgroup 1, the recommended dose for him/her is

$$
x_{k+1}=\arg \min _{x \in \mathcal{D}}\left|\psi_{1}\left(x, \hat{\beta}_{k}\right)-\theta\right| .
$$

If the $(k+1)$ th patient is in subgroup 2 , then, the recommended dose for him/her is

$$
x_{k+1}=\arg \min _{x \in \mathcal{D}}\left|\psi_{2}\left(x, \hat{\beta}_{k}, \hat{b}_{k}\right)-\theta\right| .
$$

In some cases, investigators can somehow anticipate the ordering between two prognostic subgroups in the trial, for instance, the younger patient group is known to have better tolerance to aggressive treatments. O'Quigley and Paoletti [2003] further extend the twosample CRM to account for the anticipated ordering information between the two subgroups. The main idea is to assign more aggressive starting dose to group 2 after the trial has begun with the patients in group 1, given that group 2 is known to be more robust to toxicity than group 1. In practice, such design could be realized via imposing a design restriction such that dose allocation in group 2 must be at least equal to the dose currently allocated in group 1, meanwhile allowing dose-skipping in group 2 but not in group 1.

\subsubsection{Nonparametric Dose-Finding Design with Ordered Groups}

Ivanova and Wang [2006] propose a nonparametric design to compete with the ordered twosample CRM in separately determining MTDs for two subgroups with possibly different 
susceptibility to toxicity.

The notations of this section remain the same as those for the two-sample CRM, only except for that the toxicity probability $\pi_{i j}=P\left(Y=1 \mid X=d_{j}, G=i\right)$ is assumed to be non-decreasing in doses within each group, such that $\pi_{i 1} \leq \cdots \leq \pi_{i K}$ for $i=1,2$. And it is supposed that, the observations of the first $m_{1}$ patients in group 1 and the first $m_{2}$ patients in group 2 are available yet, where group 1 has escalated to dose $d_{k_{1}}$ meanwhile group 2 has reached dose $d_{k_{2}}$. The proportion of toxicity responses at dose $d_{j}$ in group $i$ is denoted as $\hat{\pi}_{i j}$, and $\hat{\Psi}=\left\{\hat{\pi}_{i j}\right\}$ is denoted as the matrix of these proportions as follows

$$
\hat{\Psi}=\left\{\begin{array}{l}
\hat{\pi}_{11}, \cdots, \hat{\pi}_{1 k_{1}}, \mathrm{NA}, \cdots, \mathrm{NA} \\
\hat{\pi}_{21}, \cdots, \hat{\pi}_{2 k_{2}}, \mathrm{NA}, \cdots, \mathrm{NA}
\end{array}\right\}
$$

where NA means "currently not available".

If group 2 is more tolerable to the treatment than group 1 , denoted as $G_{2} \leq G_{1}$, then the bivariate isotonic regression would be performed to obtain an isotonized toxicity proportion matrix $\Psi^{(2,1)}=\left\{\pi_{i j}^{(2,1)}\right\}$. Specifically, the elements of $\Psi^{(2,1)}$ satisfy that $\pi_{2 j}^{(2,1)} \leq \pi_{1 j}^{(2,1)}$, $j=1, \cdots, K$, and $\pi_{i 1}^{(2,1)} \leq \cdots \leq \pi_{i K}^{(2,1)}, i=1,2$; meanwhile,

$$
M^{(2,1)}=\sum_{i=1}^{2} \sum_{j=1}^{K}\left(\pi_{i j}^{(2,1)}-\hat{\pi}_{i j}\right)^{2}=\min _{\tilde{\pi}_{i j} \text { satisfy } G_{2} \leq G_{1}} \sum_{i=1}^{2} \sum_{j=1}^{K}\left(\tilde{\pi}_{i j}-\hat{\pi}_{i j}\right)^{2} .
$$

Similarly, if group 1 is more tolerable than group 2, denoted as $G_{1} \leq G_{2}$, then the bivariate isotonic regression would be performed to obtain $\Psi^{(1,2)}=\left\{\pi_{i j}^{(1,2)}\right\}$ : the elements of $\Psi^{(1,2)}$ satisfy that $\pi_{1 j}^{(1,2)} \leq \pi_{2 j}^{(1,2)}, j=1, \cdots, K$, and $\pi_{i 1}^{(1,2)} \leq \cdots \leq \pi_{i K}^{(1,2)}, i=1,2$; meanwhile

$$
M^{(1,2)}=\sum_{i=1}^{2} \sum_{j=1}^{K}\left(\pi_{i j}^{(1,2)}-\hat{\pi}_{i j}\right)^{2}=\min _{\tilde{\pi}_{i j} \text { satisfy } G_{1} \leq G_{2}} \sum_{i=1}^{2} \sum_{j=1}^{K}\left(\tilde{\pi}_{i j}-\hat{\pi}_{i j}\right)^{2} .
$$

Bt comparing $M^{(2,1)}$ with $M^{(1,2)}$, the toxicity probability estimators corresponding to the 
best fit would be selected as the final estimators, that is $\Psi^{*}=\left\{\pi_{i j}^{*}\right\}$.

Then, for the $\left(m_{1}+1\right)$ th patient in group 1, the dose would be:

$$
x_{m_{1}+1}=d_{k_{1}+1} \mathrm{I}\left\{\pi_{1 k_{1}}^{*} \leq \theta-\Delta\right\}+d_{k_{1}-1} \mathrm{I}\left\{\pi_{1 k_{1}}^{*} \geq \theta+\Delta\right\}+d_{k_{1}} \mathrm{I}\left\{\theta-\Delta<\pi_{1 k_{1}}^{*}<\theta+\Delta\right\} ;
$$

Similarly, for the $\left(m_{2}+1\right)$ th patient in group 2 , the dose would be:

$$
x_{m_{1}+1}=d_{k_{2}+1} \mathrm{I}\left\{\pi_{2 k_{2}}^{*} \leq \theta-\Delta\right\}+d_{k_{2}-1} \mathrm{I}\left\{\pi_{2 k_{2}}^{*} \geq \theta+\Delta\right\}+d_{k_{2}} \mathrm{I}\left\{\theta-\Delta<\pi_{2 k_{2}}^{*}<\theta+\Delta\right\},
$$

where $\theta$ is the target toxicity rate and $\Delta \geq 0$ is a pre-specified design parameter.

Upon the trial stops as it reaches a given number of patients, the MTD in group $i$ is estimated by $d_{k(i)}$, where $k(i)=\arg \min _{1 \leq j \leq \nu_{i}}\left|\pi_{i j}^{*}-\theta\right|$ for $i=1,2$, and $\nu_{i}$ is the maximum index such that there are subjects in group $i$ assigned to dose $d_{\nu_{i}}$ but no subjects are assigned to doses above $d_{\nu_{i}}$.

\subsubsection{Individualized Continual Reassessment Method}

Piantadosi and Liu [1996] extend CRM to incorporate an ancillary PK measurement (AUC) into the adaptive dose-escalation design. AUC measures the overall exposure to a drug, and should be positively associated with the toxicity of an anti-cancer drug. The motivation of the proposed method includes that both PK information and dosage are important in predicting toxicity response, and CRM can indeed explicitly account for PK measures within dose escalation via a parametric model.

The response $Y$ is a binary toxicity indicator with a target toxicity rate $\theta$, while the dose $X$ is assumed to take values in a continuous range. The additional covariate $Z$ (AUC) is obtained from a two-compartment PK model, which postulates the transit of drug from blood to a tissue compartment and elimination from blood to have linear kinetics.

Based on the two-compartment model, there could be derived out the following PK 
relationship between AUC, dosage and the drug elimination rate $(\gamma)$ :

$$
\mathrm{AUC}=\frac{\text { Dose }}{\gamma}
$$

However, due to PK measurements are only available after patients take the treatment. Therefore, in order to adjust for AUC, the authors estimate AUC by $z_{i}=\frac{r_{0} t_{0}}{\gamma}+\epsilon_{i}$, where $r_{0}$ is the constant rate at which the drug is administered by continuous intravenous infusion, $t_{0}$ is the duration from the beginning of infusion to the stopping time that one is usually administered in the trial, and $\epsilon$ is a random error.

Given the estimated AUC, the dose-response curve is modeled in a parametric form with the logit link as follows:

$$
P\{Y=1 \mid x, z\}=\frac{\exp \left\{\beta_{0}+\beta_{1} x+\beta_{2} \Delta_{Z}\right\}}{1+\exp \left\{\beta_{0}+\beta_{1} x+\beta_{2} \Delta_{Z}\right\}},
$$

where $\Delta_{Z}=z-\frac{x}{\gamma}, \beta_{0}$ is assumed to be known, $\beta_{1}>0$ and $\beta_{2}>0$. In words, if either the dose or the residual between the estimated AUC and the true value increases, then the toxicity probability should also increase.

The authors apply the Bayesian method rather than the maximum likelihood procedure to estimate the parameters due to the small sample sizes. Based on the joint likelihood

$$
L_{n}\left(\beta_{1}, \beta_{2}\right)=\prod_{i=1}^{n}\left\{P\left\{y_{i}=1 \mid \beta_{1}, \beta_{2}, x_{i}, z_{i}\right\}^{y_{i}}\left[1-P\left\{y_{i}=1 \mid \beta_{1}, \beta_{2}, x_{i}, z_{i}\right\}\right]^{1-y_{i}}\right\}
$$

the Bayesian estimators of the parameters are as follows

$$
\begin{aligned}
& \hat{\beta}_{1}=\frac{\int_{0}^{\infty} \int_{0}^{\infty} \beta_{1} L\left(\beta_{1}, \beta_{2}\right) f\left(\beta_{1}, \beta_{2}\right) d \beta_{1} d \beta_{2}}{\int_{0}^{\infty} \int_{0}^{\infty} L\left(\beta_{1}, \beta_{2}\right) f\left(\beta_{1}, \beta_{2}\right) d \beta_{1} d \beta_{2}}, \\
& \hat{\beta}_{2}=\frac{\int_{0}^{\infty} \int_{0}^{\infty} \beta_{2} L\left(\beta_{1}, \beta_{2}\right) f\left(\beta_{1}, \beta_{2}\right) d \beta_{1} d \beta_{2}}{\int_{0}^{\infty} \int_{0}^{\infty} L\left(\beta_{1}, \beta_{2}\right) f\left(\beta_{1}, \beta_{2}\right) d \beta_{1} d \beta_{2}},
\end{aligned}
$$


where the joint prior of $\beta_{1}$ and $\beta_{2}$ was chosen to be a bivariate uniform density $f\left(\beta_{1}, \beta_{2}\right)$.

The dose escalation scheme is as follows: the first $m_{1}$ patients receive the starting dose $x_{1}$ that is chosen by the investigators according to pre-clinical studies. At the second, upon the response and AUC are observed, the parameters $\beta_{1 k}$ and $\beta_{2 k}$ will be estimated using the first $k$ observations. Then, the dose for next $m_{k+1}$ patients can be calculated by inverting the model equation.

$$
x_{k+1}=\frac{\operatorname{logit}(\theta)-\beta_{0}-\hat{\beta}_{2 k} \Delta_{Z_{k}}}{\hat{\beta}_{1 k}}
$$

The process is repeated until either the new dose no longer changes or a pre-specified sample size is reached.

In the simulation, the authors compare the individualized CRM with the traditional CRM, and find that the dose-finding scheme adjusted for PK data does not result in dramatic improvement in all circumstances, in terms of the biases of recommended doses. The proposed method is superior to CRM only when the variability of AUC estimates or the elimination rate $\gamma$ is comparatively high to that of dose. And, only when AUC data are important in predicting response and also variable enough so as to uncouple from dose, the proposed method would perform significantly better than the traditional CRM.

\subsubsection{Individualized Escalation with Overdose Control}

Babb and Rogatko [2001] extend EWOC to adjust dose levels of PNU treatment according to plasma concentration of anti-SEA antibodies for patients with advanced adenocarcinomas of gastrointestinal origin. MTD is defined as a increasing function $\nu(z)$ of the anti-SEA antibodies concentration $Z$, which is measured three days prior to the onset of PNU treatment. PNU dose level is represented by the random variable $X$, meanwhile the response is a binary toxicity indicator $Y$ with a target toxicity rate $\theta$. After observation of $k$ patients, the history data is $H_{k}=\left\{\left(x_{i}, y_{i}, z_{i}\right): 1 \leq i \leq k\right\}$.

Unlike the two-effect model employed by Piantadosi and Liu [1996], Babb and Rogatko 
[2001] propose a varying coefficient dose-response model to incorporate the covariate. Under the logit link, the toxicity probability given dose level $x$ and anti-SEA concentration $z$ is modeled as follows

$$
P\{Y=1 \mid x, z\}=\frac{\exp (a(z)+\beta(z) x)}{1+\exp (a(z)+\beta(z) x)} .
$$

By specifying the following particular forms for the coefficient functions

$$
\begin{cases}a(z)=a+\delta \ln (z), & \text { assuming } \delta<0, \\ \beta(z) \equiv \beta, & \text { assuming } \beta>0,\end{cases}
$$

then the dose-response model becomes

$$
P\{Y=1 \mid x, z\}=\frac{\exp (a+\delta \ln (z)+\beta \ln (x))}{1+\exp (a+\delta \ln (z)+\beta \ln (x))} .
$$

Based on the above model, the joint likelihood given the first $k$ th observations $H_{k}$ is

$$
L\left(a, \beta, \delta \mid H_{k}\right)=\frac{\exp \left\{a \sum_{i=1}^{k} y_{i}+\delta \sum_{i=1}^{k} y_{i} \ln \left(z_{i}\right)+\beta \sum_{i=1}^{k} y_{i} \ln \left(x_{i}\right)\right\}}{\prod_{i=1}^{k}\left[1+\exp \left\{a+\delta \ln \left(z_{i}\right)+\beta \ln \left(x_{i}\right)\right\}\right]} .
$$

Thus, given the target toxicity rate $\theta=0.1$, then MTD can be derived out as

$$
\nu(z)=\left(9 e^{a}\right)^{-1 / \beta} z^{-\delta / \beta},
$$

which indicates MTD is increasing in the anti-SEA concentration given that $\delta / \beta$ is constrained to be negative. Prior to the PNU trial, a preliminary study is necessary for finding out the permissible dose range as function of the anti-SEA concentration $z \in\left[c_{1}, c_{2}\right]$, $\Gamma(z)=\left[X_{\min }(z), X_{\max }(z)\right]$, where MTD $\nu(z)$ will be searched for.

Upon the marginal posterior distribution of $\operatorname{MTD} \nu(z)$ is derived out given $H_{k}$, which is denoted as $\pi_{z}\left(\nu \mid H_{k}\right)$ for any fixed $z \in\left[c_{1}, c_{2}\right]$, then the dose for the $(k+1)$ th patient is 


$$
\begin{aligned}
x\left(z_{k+1}\right) & =\arg \min _{x \in \Gamma\left(z_{k+1}\right)} E_{z_{k+1}}\left(l_{z_{k+1}}(x, \nu) \mid H_{k}\right) \\
& =\arg \min _{x \in \Gamma\left(z_{k+1}\right)} \int_{\Gamma\left(z_{k+1}\right)} l_{z_{k+1}}(x, \nu) \pi_{z_{k+1}}\left(\nu \mid H_{k}\right) d \nu
\end{aligned}
$$

where $E_{z_{k+1}}\left(l_{z_{k+1}}(x, \nu) \mid H_{k}\right)$ is the expected posterior loss associated with any fixed dose $x \in \Gamma\left(z_{k+1}\right)$. Like the loss used in EWOC, the asymmetric loss function is defined as, for a given $\alpha \in(0,1)$ and any fixed $z \in\left[c_{1}, c_{2}\right]$,

$$
l_{z}(x, \nu(z))= \begin{cases}\alpha(\nu(z)-x), & \text { if } x \leq \nu(z), \text { that is, if } x \text { is an underdose } \\ (1-\alpha)(x-\nu(z)), & \text { if } x>\nu(z), \text { that is, if } x \text { is an overdose }\end{cases}
$$

The loss criterion implies that, for any $\Delta>0$, the loss incur by treating a patient at $\Delta$ units above MTD is $\frac{1-\alpha}{\alpha}$ times greater than the loss associated with treating the patient at $\Delta$ units below MTD. Low value of the threshold $\alpha$ would result in a cautious escalation scheme with relatively small increments in dose, while high values would lead to a more aggressive escalation.

Consequently, by the conclusion of the PNU trial, each patient received a dose level corresponding to the best estimate of MTD given the historical data. The flexibility that the dose-finding design and dose-response modeling gain via allowing dose levels to adjust for patient-specific needs is intuitively obvious. 


\subsection{Motivations}

\subsubsection{Literature Review Discussions}

First of all, the proposals reviewed in Section 1.3 aim at a target toxicity rate of either binomial or multinomial events while adjusting for patient's risk. In fact, rather than limited to phase I designs, there is also individualized dose-finding designs developed for phase I/II trials, for instance, Thall et al. [2008] consider a Bayesian method, called EffTox, based on bivariate binary outcomes using probit models to depict the marginal dose-covariate-response relationships.

While these proposals vary in model assumptions and decision rules, they attempt to treat patients near the target dose. This type of approach, while ethically appealing, lacks theoretical justification, for instance the consistency of recommended dose sequence. In case of a homogeneous population, Cheung [2002] shows that applying the continual reassessment method with a multiparameter model may yield a dose sequence converging to a limit bounded away from the true target dose even if the model assumption is correct. The reason for this phenomenon is that the dose sequence depends on patient outcomes and is random. As such, there is no intrinsic mechanism to ensure that the design matrix will satisfy the conditions for consistent estimation of the model parameters, and hence the target dose. Therefore, we are motivated to develop an intrinsic mechanism for either traditional or individualized dose-finding designs, such that it can not only ensure consistent dose sequence but also allow for precise estimation for the parametric dose-response models.

At the second, most existing individualized dose-finding designs are proposed for the studies of traditional cytotoxic agents, where the primary endpoints are most often binary toxicity responses. In the past decades, an emphasis shift from the traditional cytotoxic agents to novel molecular targeted therapies in anti-cancer drug development has necessitated the development of new phase I studies that considers a new type of primary endpoints. 
Because, most molecular targeted agents are more selective and less toxic than cytotoxic agents; and, the fundamental assumption that toxicity-based dosing methods rely on, that the drug's therapeutic and toxic effects of the drug increase in parallel as the dose is escalated, could be violated for the molecular targeted agents [Booth et al., 2008]. At such cases, the traditional binary toxicity response might not be an ideal primary endpoint for model-based dose-finding approaches. Instead, new types of ideal primary endpoints should be considered for phase I cancer trials, for example the PK responses due to their close connection to both the dosage and safety endpoints [Fox et al., 2002].

So far, several approaches have been proposed to target at the expected value of a continuous outcome. For example, O'Quigley et al. [2010] consider PK endpoint using an extended continual reassessment method; Ivanova and Kim [2009] aim at a given mean of AGT protein in a glioma study based on t-statistics. To address multiple toxicities, Bekele and Thall [2004] propose a Bayesian design that targets at the mean of a numerical toxicity burden; in the sequel, Bekele et al. [2010] extend the idea to risk-specific dosing using average toxicity score. However, except for Bekele et al. [2010], which model toxicity within each risk group, these previous works do not account for inter-patient heterogeneity, especially for a continuous prognostic covariate. Thus, it is in need to develop the corresponding methods for continuous PK responses while adjusting for inter-patient heterogeneity.

Moreover, many parametric model based dose-finding designs assume a known link function with a location and scale parameters between the toxicity responses and dosage, for example the logistic link for CRM in Section 1.2.2. In real practices, the true links are never known, however, the methods that are robust to link misspecification are seldom developed for phase I dose-finding designs. This important but neglected problem has drawn recent focus from researchers in phase I community, such as Warfield and Roy [2013]. For the case of binary toxicity responses and discrete dose levels, Warfield and Roy [2013] propose a semiparametric sequential dosing algorithm that aims at estimating the maximum tolerated 
dose (MTD) with being robust against link misspecification. By assuming the unknown link function is a cumulative distribution function from a continuous location-scale family, their proposal essentially incorporates a univariate nonparametric binary regression into a sequential D-optimal design, but cannot be applied to adjust for additional prognostic covariates. At present, for individualized dosing approaches, the robust designs can adapt for more general model settings with unknown dose-response link functions are missing and worth efforts in explorations.

\subsubsection{Motivating Trial: the Irinotecan Study}

Irinotecan is a topoisemerase I inhibitor that is approved as a first and second line treatment of metastatic colorectal cancer. The metabolism of irinotecan and exposure to its active metabolite SN-38 are influenced by several enzymes such as CYP3A4, whose expression and function could be affected by numerous environmental and genetic factors [Mathijssen et al., 2004]. Therefore, in the presence of large PK variability of metabolizing irinotecan among the patient population, the traditional "one-size-fit-all" dosing concept could cause severe ethical issues resulted from either overdosing or underdosing. For instance, on one hand, if a subject whose ability of eliminating irinotecan is low but was treated at a high dosage, then he/she could experience severe toxicity events; on the other hand, if a subject who has strong ability of eliminating irinotecan but was treated at a low dosage, then he/she would never receive sufficient treatment efficacy.

In order to control for inter-patient PK variability in dose determination, van der Bol et al. [2010] compare two individualized dosing algorithms in a two-arm irinotecan study. They use irinotecan as a single agent in 40 patients with metastatic colorectal cancer: 20 patients are randomized to receive doses via an equation algorithm based on a single compartment model, and the other 20 are dosed by the conventional body surface area (BSA) formula. The primary endpoint of the irinotecan study is a continuous PK response (AUC), which 
measures a patient's overall exposure to irinotecan. The dosage of irinotecan is continuous, meanwhile there is also a continuous PK covariate (clearance), which measures individual ability of eliminating a drug.

More precisely, let $y$ be the logarithm of AUC of irinotecan, $x$ be the logarithm of irinotecan dose, and $z^{*}$ be the logarithm of irinotecan clearance. A single compartment model states a PK relationship between AUC, dose and clearance such that

$$
y=x-z^{*}
$$

Since the study aims to achieve an AUC level of $22.157 \mu \mathrm{g} \times \mathrm{h} / \mathrm{mL}$, based on the above PK relationship, the equation based algorithm [van der Bol et al., 2010] prescribes a dose according to the formula

$$
x=t_{0}+z
$$

where $t_{0}=\log (22.157)$ and $z$ is the predicted clearance on log-scale based on the patient's phenotypes, including $\gamma$-glutamyltransferase, midazolam activity, and height [Ratain and Innocenti, 2010]. Since the clearance as a PK measurement is only available after treatment, van der Bol et al. [2010] use the predicted clearance instead in the equation dosing algorithm (1.3). The rationale is that patients with higher predicted irinotecan clearance can tolerate and should receive higher dose, so as to reach a target plasma concentration. As the control arm, the patients treated according to the conventional BSA formula receive doses based on

$$
x=\log (350)+\mathrm{B}
$$

where $B$ is the logarithm of the body surface area of the patient.

As a result, van der Bol et al. [2010] find that the incorporation of predicted clearance in dosing algorithm can lower the incidence of toxicity events. The descriptive statistics of 
irinotecan study are summarized in Table 1.2. The irinotecan data will be deeply utilized in the simulation studies of this dissertation, and the BSA and equation based dosing algorithms will be included in the design comparisons as individualized dosing approaches in present clinical practices.

Table 1.2: Summary of the Irinotecan Data

\begin{tabular}{ccccccll}
\hline \hline & & & & & \multicolumn{3}{c}{ Correlation with Significance (p-value) } \\
\cline { 7 - 8 } Variable & Mean & Median & S.D. & Range & $\mathrm{x}$ & $\mathrm{z}$ & $\mathrm{B}$ \\
\hline $\mathrm{y}$ & 3.05 & 3.01 & 0.26 & {$[2.56,3.76]$} & $0.12(0.47)$ & $-0.10(0.53)$ & $-0.09(0.60)$ \\
$\mathrm{x}$ & 6.49 & 6.49 & 0.20 & {$[5.94,6.97]$} & & $0.67(0.00)$ & $0.63(0.00)$ \\
$\mathrm{z}$ & 3.44 & 3.46 & 0.26 & {$[2.83,3.85]$} & & & $0.53(0.00)$ \\
$\mathrm{B}$ & 0.63 & 0.63 & 0.12 & {$[1.36,2.37]$} & & & \\
\hline
\end{tabular}

\subsubsection{Summary}

In brief, motivated by the limitations of existing individualized dosing methods and the irinotecan study, this dissertation research develops two novel individualized dose-finding designs for phase I cancer trials, the repeated least squares procedure and semiparametric link-adapted recursion. Both designs are proposed for the cases with continuous PK responses and continuous dosage, meanwhile there exists significant inter-individual heterogeneity in the ability of metabolizing a drug.

Under linear dose-response models, the proposed repeated least squares procedure determines dose according to individual ability of metabolizing a drug, the same time an intrinsic mechanism guarantees the consistency of model parameter estimates and recommended dose sequence. It sequentially applies least squares fit to estimate the dosing function, subject to a consistency constraint based on an eigenvalue theory in the stochastic linear regression established by Lai and Wei [1982] and Wei [1985].

The second major goal of this research is to develop a dosing method that can not only adjusts for inter-individual PK variability, but is also robust to the misspecification of dose-response link function. Thus, we further extend the linear model to a more general 
semiparametric model setting, where the response is associated with the linear combination between dose and covariate via an unknown nonlinear link function. Under this model, a novel semiparametric design is proposed to recursively estimates the dosing function by utilizing an iterative semiparametric regression approach to estimate the link function and direction parameter, so as to attain enhanced robustness against model misspecification.

The rest of this dissertation are organized as follows. Chapter 2 introduces the methods of the repeated least squares procedure, which includes two distinct designs, respectively without and with a consistency constraint. Chapter 3 introduces the calibration procedure for the constrained design, and presents simulation studies on comparing two formula-based individualized dosing algorithms with the proposed repeated least squares procedure under the linear models. Chapter 4 extends the linear model to a semiparametric model, introduces the method of our proposed semiparametric link-adapted recursion, and then presents simulation studies on the comparison between the repeated least squares procedure and semiparametric design in terms of dosing accuracy, estimation precision and robustness to model misspecification. At last, Chapter 5 summarizes the contributions of this research and discusses on its potential further extension work. 


\section{Chapter 2}

\section{Repeated Least Squares Procedure}

\subsection{Linear Dose-Response Model}

Throughout the rest of this dissertation, the notations will remain the same as those in Section 1.4.2, such that $y$ is the logarithm of irinotecan AUC with the target level $t_{0}=$ $\log (22.157), x$ is the logarithm of irinotecan dose, $z$ is the predicted clearance on log-scale, and $B$ is the logarithm of body surface area (BSA).

The dosing algorithm (1.3) by [van der Bol et al., 2010] assumes that the predicted clearance $z$ is a perfect estimate of the true clearance $z^{*}$, which is not observable at baseline, and that the PK characterization of irinotecan behaves according to the single compartment model. While the study data show a plausible linear relationship between $z^{*}$ and $z$, that is, $z^{*}=\psi_{0}+\psi_{1} z+\epsilon^{*}$, there is strong evidence to support that $\psi_{0} \neq 0$ and $\psi_{1} \neq 1$, as the p-values of corresponding Wald tests are 0.023 and 0.024 respectively (see Figure 1(a)).

Motivated by both the theoretical PK relationship (1.2) and the linear relationship between $z^{*}$ and $z$, we propose a linear regression model to describe the relationship between the AUC, dose and predicted clearance on the log-scale:

$$
y=\alpha+\beta x+\gamma z+\epsilon
$$


where $\epsilon$ is random noise with mean 0 and finite variance $\sigma^{2}$.

The PK relationship (1.2) derived from a single compartment model would be a special case of model $(2.1)$ with $(\alpha, \beta, \gamma)=(0,1,-1)$ (see Figure 1(b)). However, the least squares fit to irinotecan data leads to an estimate $(\hat{\alpha}, \hat{\beta}, \hat{\gamma})=(1.26,0.46,-0.34)$ and suggests that $(\alpha, \beta, \gamma) \neq(0,1,-1)$ is significant (Wald test $\mathrm{p}$-value $=0.026)$.

Figure 2.1: Fitting a Linear Model to the Irinotecan Data

(a) Fitted Linear Model of $\mathrm{z}^{*}$ on $\mathrm{z}$

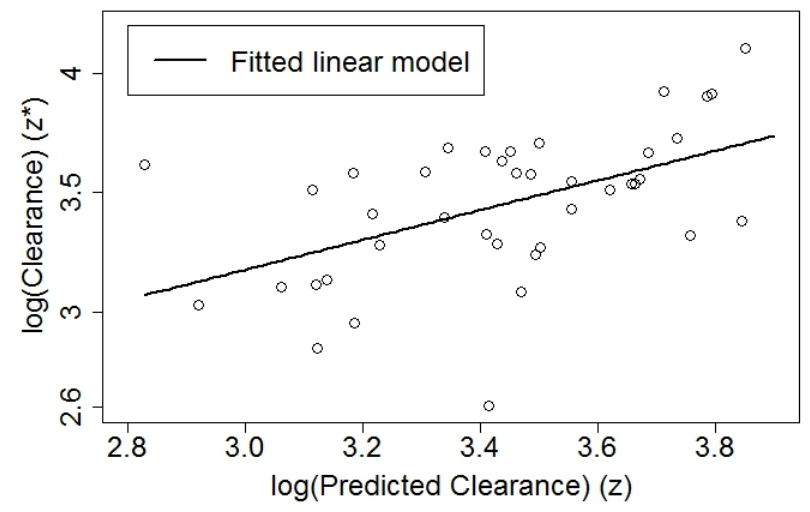

(c) Fitted Linear Model of $y$ on $x$ and $z$

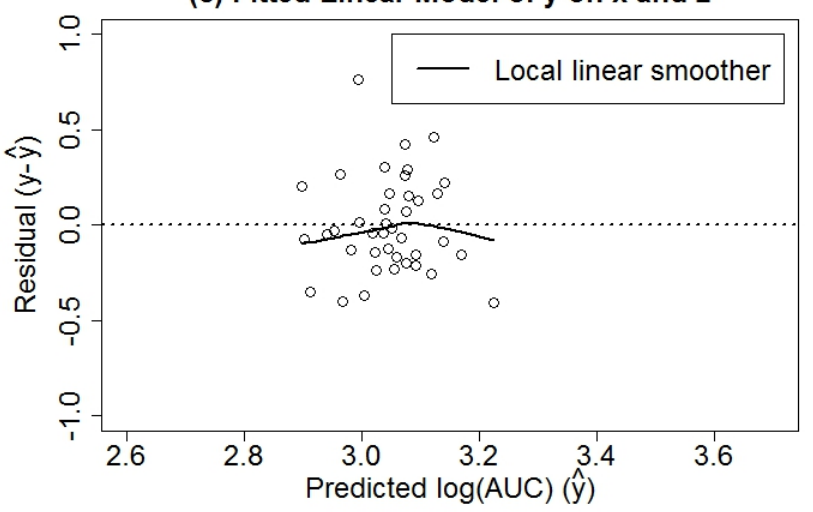

(b) Equation Method ( $y=x-z)$

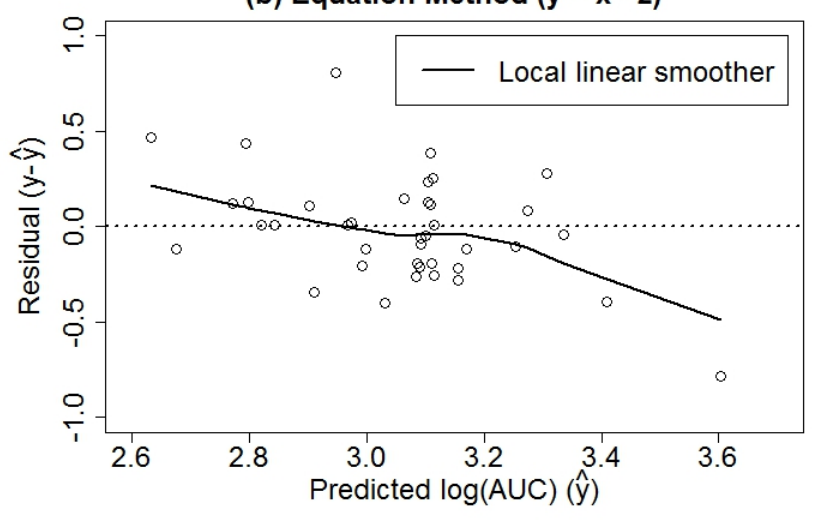

(d) Dosing Algorithm

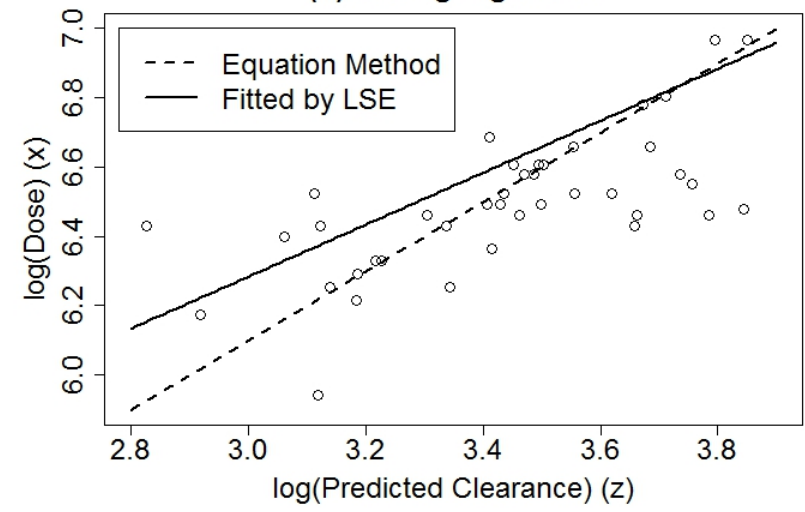

In contrast, the linear model (2.1) appears to be more adequate for the irinotecan data, compared to PK model (1.2). The residuals of single compartment model (see Figure 2.1(b)) are slightly larger than those of least squares fit (see Figure 2.1(c)); and, the residuals of single compartment model generally decreases as fitted $y$ grows, which pattern is shown by a nonparametric smoothing curve between the residual and fitted $y$, however, the residuals of least squares fit show no dependency on their fitted $y$. In fact, Figure 1(d) shows that 
the equation dosing algorithm (1.3) assigns lower doses than the that estimated by the least squares fit, $x=\left(t_{0}-1.26-0.34 z\right) / 0.46$.

Based on the linear dose-response model (2.1), two individualized dose-finding designs are developed for phase I cancer trials with continuous PK responses and dosage.

\subsection{Unconstrained Repeated Least Squares Design}

For an individualized phase I cancer trial, the trial objective is to estimate a patient-specific dose $x=\theta(z)$ so that $E(y \mid x, z)$ can achieve the target level $t_{0}$, rather than the estimation of MTD for the traditional phase I cancer trials. Under the model (2.1), this goal would be equivalent to estimate the following dosing function

$$
\theta(z)=\frac{t_{0}-\alpha-\gamma z}{\beta}, \quad \text { for any given } z \in I_{z}
$$

where $I_{z}=\left[z_{\min }, z_{\max }\right]$ is the proper range of $z$ and $\theta(z)$ should take value within a prespecified dose range of $x$, that is $I_{x}=\left[x_{\min }, x_{\max }\right]$.

The estimation of dosing function $\theta(z)$ involves the estimation of coefficients of model (2.1). Thus, it would be natural to apply the least squares fit to obtain the estimates of

$(\alpha, \beta, \gamma)$, and then substitute them into $\theta(z)$. Due to the sequential manner of the design, the estimation stated above is then conducted recursively as the trial proceeds. This is indeed the main idea of the proposed repeated least squares (RLS) design.

\section{Algorithm 1 (RLS)}

1. For the $(i+1)$ th subject, we at first estimate $\theta(z)$ by

$$
\hat{\theta}_{i}(z)=\frac{t_{0}-\hat{\alpha}_{i}-\hat{\gamma}_{i} z}{\hat{\beta}_{i}}, \text { for any fixed } z \in I_{Z}
$$


where $\left(\hat{\alpha}_{i}, \hat{\beta}_{i}, \hat{\gamma}_{i}\right)^{\prime}$ is the least squares estimate using observations of the first $i$ subjects;

2. Then, the dose for the $(i+1)$ th subject is $x_{i+1}=\min \left\{\max \left\{\hat{\theta}_{i}\left(z_{i+1}\right), x_{\min }\right\}, x_{\max }\right\}$;

3. Once $y_{i+1}$ is observed, the least squares estimate of $(\alpha, \beta, \gamma)$ will be updated; the trial continues with repeating Step 1 through 3 until a pre-specified sample size is reached.

It is worth to mention that, so as to ensure that the recommended doses take value within the proper range $I_{x}$, the estimate doses are truncated by the lower and upper limits of the dose range. At the trial's end, an estimated dosing function $\hat{\theta}_{n+1}(z)$ will be recommended to future patients.

The repeated least squares design is a straightforward approach to determine dose with respect to individual predicted clearance under model (2.1). It is actually an extension of the maximum likelihood recursion method [Wu, 1986] in the presence of an extra covariate. The simplicity of RLS can give it good feasibility in practices, however, RLS does not necessarily result in desirable estimation properties of the dosing function, because it is unable to exclude the occurrence of asymptotic co-linearity to its design matrix.

Due to ethical issues, the primary concern on the theoretical properties of a dose-finding design is the dosing accuracy over a trial. A consistent dose sequence, which is defined as following, can ensure that the assigned doses would eventually approach the true dosing function in a certain sense, and so avoid systematic dosing biases to happen during a trial.

Definition 2 (Strong Consistency of Dose Sequence) The recommended dose sequence is strongly consistent for the true doses, if

$$
\left|x_{n}-\theta\left(z_{n}\right)\right| \stackrel{\text { a.s. }}{\longrightarrow} 0, \text { as } n \rightarrow \infty \text {. }
$$

It can be shown that, as long as the strong consistency of least squares estimator $\left(\hat{\alpha}_{i}, \hat{\beta}_{i}, \hat{\gamma}_{i}\right)^{\prime}$ holds, the estimated dosing function (2.3) converges point-wisely to the true dosing function 
(2.2) by the Continuous Mapping theorem, and thus the strong consistency (2.4) can be further guaranteed.

However, the convergence rate of dose sequence can actually in turn affect the strong consistency of the least squares estimator. If doses converge to some function too quickly, it would cause columns of the design matrix to asymptotically have co-linearity. For simplicity, if we consider the scenario with dose only but no other covariate, then a rapidly converging dose sequence would cause that most dose values concentrate closely around a constant and the dose column is nearly co-linear with the intercept column. This phenomenon is described as the asymptotic singularity by Chen et al. [1999], which results in poor least squares estimation essentially because the design fails to provide sufficient "information". The strong consistency (2.4) controls overall dose estimation precision of a trial and excludes that systematic dosing errors happen to all subjects. Therefore, the dose convergence rate of RLS needs to be controlled in order to eliminate the asymptotic singularity.

\subsection{Repeated Least Squares with Eigenvalue Constraint Design}

Under the stochastic linear regression models, Lai and Wei [1982] establish a sufficient condition for the strong consistency of least squares estimator, which is the following eigenvalue condition:

$$
\frac{\log \lambda_{\max }(n)}{\lambda_{\min }(n)} \stackrel{\text { a.s. }}{\longrightarrow} 0, \quad \text { as } n \rightarrow \infty
$$

where $\lambda_{\max }(n)$ and $\lambda_{\min }(n)$ are respectively the maximum and minimum eigenvalues of $M_{n}^{\prime} M_{n}$, and $M_{n}$ is the design matrix of the first $n$ subjects in least squares fit. Lai and Wei [1982] point out by an example that, if the eigenvalue condition (2.5) is violated, the least squares estimator would fail to be strongly consistent. This theorem along with the model 
settings, as well as the example and proof sketch are summarized in Section 2.5.

The essential reason of the inconsistency is the asymptotic singularity resulted from the uncontrolled dose convergence rate. The eigenvalue condition (2.5) in effect slows down the dose convergence rate by requiring $\lambda_{\min }(n)$ to diverge faster than $\log \lambda_{\max }(n)$. As Frees and Ruppert [1990] state that, spreading the design points so that they do not concentrate around a target too rapidly can improve the precision of least squares estimation. Thus, the importance of the eigenvalue condition (2.5) is to help the design gain sufficient information by spreading design points apart, and thus eliminate the occurrence of asymptotic singularity and guarantee precision of least squares estimator.

In general, without any control on the dose convergence rate, model based dose-finding designs, such as RLS, do not necessarily result in a strongly consistent least squares estimator. Therefore, a general principle is in need for ensuring estimation precision of a dose-finding design. The eigenvalue condition (2.5) is such a principle.

Motivated by the eigenvalue condition (2.5), we propose the repeated least squares with eigenvalue constraint (RLSEVC) design.

\section{Algorithm 2 (RLSEVC)}

1. For the $(i+1)$ th subject, we estimate $\theta(z)$ by $\hat{\theta}_{i}(z)=\frac{t_{0}-\hat{\alpha}_{i}-\hat{\gamma}_{i} z}{\hat{\beta}_{i}}$, for any given $z \in I_{z}$

2. Determine the dose $x_{i+1}=\underset{x \in I_{x}}{\arg \min }\left|x-\hat{\theta}_{i}\left(z_{i+1}\right)\right|$ subject to an eigenvalue constraint

$$
\rho_{i+1}\left(x, z_{i+1}\right) \leq r_{i+1},
$$

where the eigenvalue ratio $\rho_{n}(x, z)=\frac{\log \lambda_{\max }(n)}{\lambda_{\min }(n)}$ is a function of $x$ and $z$, and the real sequence $r_{n}$ is specified in form of $r_{n}=C n^{-\delta_{1}}(\log n)^{1-\delta_{2}}$.

3. Upon $y_{i+1}$ is observed, the least squares estimate of $(\alpha, \beta, \gamma)$ will be updated; the trial 
continues with repeating Step 1 through 3 until a pre-specified sample size is reached.

At the end of the trial, the estimated dosing function $\hat{\theta}_{n+1}(z)$ will be recommended to future patients.

Particularly, $C$ is a positive constant determined by an initial design, which will be introduced in details in Section 3.3. The convergence rate of eigenvalue constraint is controlled by the design parameter $\left(\delta_{1}, \delta_{2}\right)$, which is selected from $\mathcal{R}=[0,1) \times[0,+\infty) \backslash 0 \times[0,1]$ to ensure that $r_{n}$ converges to 0 . The form of the real sequence $r_{n}$ is actually not unique, as long as $r_{n}$ converges to 0 . We select this specific form

$$
r_{n}=\frac{C \log n}{n^{\delta_{1}}(\log n)^{\delta_{2}}}=C n^{-\delta_{1}}(\log n)^{1-\delta_{2}}
$$

under motivation of the eigenvalue condition (2.5). The numerator $\log n$ is the typical rate at which the maximum eigenvalue $\lambda_{\max }(n)$ in logarithm diverges to $\infty$, according to Theorem 2 of Wei [1985]; meanwhile, the denominator of $r_{n}$ is chosen to explicitly control the rate at which it diverge faster than $\log n$.

By construct, each single dose suggested by RLSEVC satisfies the eigenvalue condition (2.5). Thus, RLSEVC naturally guarantees the strong consistency of least squares estimator and hence that of the estimated dosing function.

\subsection{Coherence Restriction}

The concept of coherence is introduced to phase I dose-finding designs by Cheung [2005] in the context of dose-escalation among a discrete range. Specifically, an escalation for a new patient is defined to be coherent only when the previous patient does not experience toxicity and a de-escalation is coherent only when previous toxicity is observed.

The coherence is a general principal to enhance practicality of a dose-finding design, under 
the motivation of ethical concerns in trial conduct. It is worth to be extended to designs for individualized dosing. However, unlike the traditional dose-escalation, not only the response of previous patient influences dose increase or decrease, but should the covariate value of current patient also impacts enhancement or reduction in dose compared to the previous. To account for both the influence of previous response and current covariate on dosage, we propose a coherence criterion for individualized dose-finding designs in phase I cancer trials as following.

Definition 3 (Coherence Restriction) For the $(i+1)$ th subject, the following restriction is applied to the individualized dose-finding procedure:

- If $y_{i}>t_{0}$ and $z_{i} \geq z_{i+1}$, then $x_{i+1} \in\left[x_{m i n}, x_{i}\right]$;

- If $y_{i}>t_{0}$ and $z_{i}<z_{i+1}$, then $x_{i+1} \in\left[x_{\min }, x_{\max }\right]$;

- If $y_{i} \leq t_{0}$ and $z_{i} \geq z_{i+1}$, then $x_{i+1} \in\left[x_{\min }, x_{\max }\right]$;

- If $y_{i} \leq t_{0}$ and $z_{i}<z_{i+1}$, then $x_{i+1} \in\left[x_{i}, x_{\text {max }}\right]$;

where a subject is considered to have toxicity if his/her $y_{i}$ exceeds the threshold $t_{0}$.

For the irinotecan trial, the dose increase is coherent when the previous subject has no toxicity and his/her predicted clearance is lower than the current subject's; the dose reduction is coherent when the previous subject has toxicity and his/her predicted clearance is higher than the current subject's. However, the definition of coherence becomes subtle and complex, when the previous subject with lower predicted clearance has toxicity, or the previous subject with higher predicted clearance has not experienced toxicity. For instance, at the former case, even though the previous subject had toxicity, a higher dose could be still safe for the current subject due to his/her stronger ability of eliminating irinotecan. Therefore, for these two scenarios, no coherence restriction is imposed on dose-finding. It is this freedom of individualized dosing that potentially benefits patients with higher efficacy 
and safety, because, in dose-determination for the current patient, previous outcomes are no longer the only consideration, drug metabolizing capability of this patient will also play an equally important role in the determination.

For RLSEVC, the eigenvalue constraint is the key to ensure an precisely estimated dosing function, while the coherence restriction can further improve dosing accuracy without sacrificing estimation precision; however, for RLS, the coherence restriction might only improve dosing cost in the absence of asymptotic singularity.

\subsection{The Eigenvalue Theorem and An Example}

In this section, the eigenvalue theory by Lai and Wei [1982] will be briefly stated at first. Then, an example will be showed that, in the presence of stochastic regressors, the least squares estimator could fail to be strongly consistent when the eigenvalue condition (2.5) gets violated.

Lai and Wei [1982] consider the following stochastic linear regression model,

$$
y_{n}=\beta_{1} x_{n 1}+\cdots+\beta_{p} x_{n p}+\epsilon_{n}, \text { for } n=1,2, \cdots
$$

where $\left\{\epsilon_{n}\right\}$ is a martingale difference sequence with respect to an increasing sequence of $\sigma$-fields $\left\{\mathcal{F}_{n}\right\}$, and $\mathbf{x}_{n}=\left(x_{n 1}, \cdots, x_{n p}\right)^{\prime}$ is the vector of sequentially determined stochastic regressors at the $n$th stage, such that $\mathbf{x}_{n}$ depends on all the previous observations $\left\{\left(\mathbf{x}_{i}, y_{i}\right)\right\}_{i=1}^{n-1}$ through certain sequential design, in other words $\mathbf{x}_{n}$ is $\mathcal{F}_{n-1}$-measurable.

Lemma 1 (Theorem 1 of Lai and Wei [1982]) Under the model (2.7), suppose that $\left\{\epsilon_{n}\right\}$ is a martingale difference sequence with respect to an increasing sequence of $\sigma$-fields $\left\{\mathcal{F}_{n}\right\}$ such that

$$
\sup _{n} E\left(\left|\epsilon_{n}\right|^{q} \mid \mathcal{F}_{n-1}\right)<\infty \text { a.s., for some } q>2 \text {. }
$$


Moreover, if the design levels $x_{n 1}, \cdots, x_{n p}$ at stage $n$ are $\mathcal{F}_{n-1}$-measurable random variables such that

$$
\lambda_{\text {min }}(n) \stackrel{\text { a.s. }}{\longrightarrow} \infty, \text { and } \frac{\log \lambda_{\max }(n)}{\lambda_{\min }(n)} \stackrel{\text { a.s. }}{\longrightarrow} 0 \text {, as } n \rightarrow \infty,
$$

where $\lambda_{\max }(n)$ and $\lambda_{\min }(n)$ are respectively the maximum and minimum eigenvalues of $\mathbf{X}_{n}^{\prime} \mathbf{X}_{n}$, and $\mathbf{X}_{n}=\left(\mathbf{x}_{1}, \cdots, \mathbf{x}_{n}\right)^{\prime}$ is the design matrix at stage $n$, then the least squares estimate $\hat{\mathbf{b}}_{n}=\left(\hat{b}_{n 1}, \cdots, \hat{b}_{n p}\right)^{\prime}=\left(\mathbf{X}_{n}^{\prime} \mathbf{X}_{n}\right)^{-1} \mathbf{X}_{n}^{\prime} Y_{n}$ converges to $\beta=\left(\beta_{1}, \cdots, \beta_{p}\right)^{\prime}$ almost surely; and, in fact,

$$
\max _{1 \leq j \leq p}\left|\hat{b}_{n j}-\beta_{j}\right|=O\left(\left\{\frac{\log \lambda_{\max }(n)}{\lambda_{\min }(n)}\right\}^{1 / 2}\right) \text { a.s. } .
$$

At most time, for sequential designs such as the stochastic approximation $(1.1), \lambda_{\max }(n)=$ $O(n)$ almost surely, which is also shown by the Theorem 2 of Wei [1985]. Especially for our proposed repeated least squares procedures, only the dose is a stochastic regressor while the realizations of the other covariate are randomly sampled from a normal distribution, thus $\lambda_{\max }(n)$ should diverge typically at the rate $n$ as the case of completely randomized designs. Therefore, as in later literature such as Chen et al. [1999], the eigenvalue condition (2.5) only requires the eigenvalue ratio between $\log \lambda_{\max }(n)$ and $\lambda_{\min }(n)$ to converge to zero almost surely, because it also naturally requires that $\lambda_{\min }(n) \stackrel{\text { a.s. }}{\longrightarrow} \infty$.

As pointed out by Lai and Wei [1982], an important case of such martingale difference sequence $\left\{\epsilon_{n}\right\}$ is independent random variables with zero mean. Thus, under our proposed model (2.1), the independent and identically distributed normal random noises $\left\{\epsilon_{n}\right\}$ obvi-

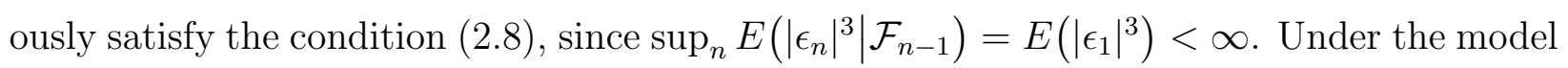
(2.1), if the $\sigma$-field $\left\{\mathcal{F}_{n}\right\}$ is generated by $\left\{\left(B_{i}, z_{i}, \epsilon_{i}\right)\right\}_{i=1}^{n}$, then $x_{n}$ is $\mathcal{F}_{n-1}$-measurable. Therefore, if the eigenvalue condition (2.5) holds, then, according to the Theorem 1 of Lai and Wei [1982], the least squares estimate $\left(\hat{\alpha}_{n}, \hat{\beta}_{n}, \hat{\gamma}_{n}\right)^{\prime}$ is strongly consistent for $(\alpha, \beta, \gamma)^{\prime}$.

\section{Example 1 (What if the Eigenvalue Condition is Violated)}


This example demonstrates that, if a sequential design does not have special control for the dose convergence rate so that the eigenvalue condition (2.5) gets violated, then the recursive least squares estimator could be inconsistent for the true model coefficients.

The following linear model is considered in this example:

$$
y_{i}=\alpha+\beta x_{i}+\epsilon_{i} .
$$

where the random errors $\epsilon_{i}$ are independent and identically distributed with zero mean and unit variance. The $\sigma$-field generated by $\epsilon_{1}, \cdots, \epsilon_{n}$ is denoted as $\mathcal{F}_{n}=\sigma\left(\epsilon_{1}, \cdots, \epsilon_{n}\right)$. The stochastic regressor $x_{i}$ is defined inductively by

$$
x_{1}=0, \quad x_{n+1}=\bar{x}_{n}+c \bar{\epsilon}_{n}, \quad n \geq 1,
$$

where $c \neq 0$ is a real constant.

The least squares estimates of $\alpha$ and $\beta$ are respectively,

$$
\begin{aligned}
\hat{\beta}_{n} & =\frac{\sum_{i=1}^{n}\left(x_{i}-\bar{x}_{n}\right) y_{i}}{\sum_{i=1}^{n}\left(x_{i}-\bar{x}_{n}\right)^{2}}=\frac{\sum_{i=1}^{n}\left(x_{i}-\bar{x}_{n}\right)\left(\alpha+\beta x_{i}+\epsilon_{i}\right)}{\sum_{i=1}^{n}\left(x_{i}-\bar{x}_{n}\right)^{2}} \\
& =\beta+\frac{\sum_{i=1}^{n}\left(x_{i}-\bar{x}_{n}\right) \epsilon_{i}}{\sum_{i=1}^{n}\left(x_{i}-\bar{x}_{n}\right)^{2}}, \\
\hat{\alpha}_{n} & =\bar{y}_{n}-\hat{\beta}_{n} \bar{x}_{n}=\alpha+\left(\beta-\hat{\beta}_{n}\right) \bar{x}_{n}+\bar{\epsilon}_{n} .
\end{aligned}
$$

According to Example 1 of Lai and Wei [1982], the sequential design leads to an asymptotically co-linear design matrix such that the eigenvalue condition (2.5) is violated, and both $\hat{\alpha}_{n}$ and $\hat{\beta}_{n}$ fail to be strongly consistent for $\alpha$ and $\beta$ respectively. The sketch of the proof is as follows.

By Corollary 1 of Lai and Robbins [1979] and Lemma 1 of Lai and Robbins [1981],

$$
\sum_{i=1}^{n}\left(x_{i}-\bar{x}_{n}\right)^{2}=\sum_{i=2}^{n} \frac{i-1}{i}\left(x_{i}-\bar{x}_{i-1}\right)^{2}=c^{2} \sum_{i=2}^{n} \frac{i-1}{i} \bar{\epsilon}_{i-1}^{2} \sim c^{2} \log n \text { a.s. }
$$


Note that

$$
\sum_{i=1}^{n}\left(x_{i}-\bar{x}_{n}\right) \epsilon_{i}=\sum_{i=2}^{n} \frac{i-1}{i}\left(x_{i}-\bar{x}_{i-1}\right)\left(\epsilon_{i}-\bar{\epsilon}_{i-1}\right)=c \sum_{i=2}^{n} \frac{i-1}{i} \bar{\epsilon}_{i-1}\left(\epsilon_{i}-\bar{\epsilon}_{i-1}\right)
$$

and $\left\{\sum_{i=1}^{n} \frac{i-1}{i} \bar{\epsilon}_{i-1} \epsilon_{i}, \quad n \geq 1\right\}$ is a martingale relative to the $\sigma$-fields $\mathcal{F}_{n}$, based on the Strong Law of Martingales (see Theorem 2 of Chow [1960]), it can be proved that

$$
\frac{1}{\log n} \sum_{i=1}^{n} \frac{i-1}{i} \bar{\epsilon}_{i-1} \epsilon_{i} \stackrel{\text { a.s. }}{\longrightarrow} 0
$$

By (2.15), (2.16) and (2.17), we can obtain that

$$
\begin{aligned}
\frac{\sum_{i=1}^{n}\left(x_{i}-\bar{x}_{n}\right) \epsilon_{i}}{\sum_{i=1}^{n}\left(x_{i}-\bar{x}_{n}\right)^{2}} & =\frac{\sum_{i=2}^{n} \frac{i-1}{i} \bar{\epsilon}_{i-1}\left(\epsilon_{i}-\bar{\epsilon}_{i-1}\right)}{c \sum_{i=2}^{n} \frac{i-1}{i} \bar{\epsilon}_{i-1}^{2}} \\
& =\frac{\sum_{i=2}^{n} \frac{i-1}{i} \bar{\epsilon}_{i-1} \epsilon_{i}}{c \sum_{i=2}^{n} \frac{i-1}{i} \bar{\epsilon}_{i-1}^{2}}-\frac{1}{c} \\
& \sim \frac{\sum_{i=2}^{n} \frac{i-1}{i} \bar{\epsilon}_{i-1} \epsilon_{i}}{c \log n}-\frac{1}{c} \text { a.s. } \\
& \stackrel{\text { a.s. }}{\longrightarrow}-\frac{1}{c}
\end{aligned}
$$

Combining (2.13) and (2.18), we can get that $\hat{\beta}_{n} \stackrel{\text { a.s. }}{\longrightarrow} \beta-\frac{1}{c}$.

Then, we will show that $\hat{\alpha}_{n}$ also fails to be strongly consistent for $\alpha$. By induction, we can show that $x_{n+1}=c \sum_{i=1}^{n} \frac{\epsilon_{i}}{i}$. Now, suppose that it holds for $x_{n}$, then

$$
\begin{aligned}
x_{n+1} & =\bar{x}_{n}+c \bar{\epsilon}_{n}=\frac{c}{n} \sum_{i=2}^{n}\left(\sum_{j=1}^{i-1} \frac{\epsilon_{j}}{j}\right)+\frac{c}{n} \sum_{i=1}^{n} \epsilon_{i} \\
& =\frac{c}{n}\left(n \epsilon_{1}+\frac{n}{2} \epsilon_{2}+\frac{n}{3} \epsilon_{3}+\cdots+\frac{n}{n-1} \epsilon_{n-1}+\epsilon_{n}\right) \\
& =c \sum_{i=1}^{n} \frac{\epsilon_{i}}{i}
\end{aligned}
$$


Given that $\left\{\frac{\epsilon_{i}}{i}\right\}$ are independent with $E\left(\frac{\epsilon_{i}}{i}\right)=0$ and $\sum_{i=1}^{\infty} \operatorname{Var}\left(\frac{\epsilon_{i}}{i}\right)=\sum_{i=1}^{\infty} \frac{1}{i^{2}}<+\infty$, by the corollary of the Kolmogorov's Three Series Theorem, $\sum_{i=1}^{\infty} \frac{\epsilon_{i}}{i}<+\infty$ a.s..

Moreover, the Strong Law of Large Numbers leads to that $\bar{\epsilon}_{n} \stackrel{\text { a.s. }}{\longrightarrow}$ 0. Then, by (2.12),

$$
\bar{x}_{n}=x_{n+1}-c \bar{\epsilon}_{n} \stackrel{\text { a.s. }}{\longrightarrow} \sum_{i=1}^{\infty} \frac{\epsilon_{i}}{i} .
$$

If we denote $S=\sum_{i=1}^{\infty} \frac{\epsilon_{i}}{i}<+\infty$ a.s., then by $(2.14)$ and $\hat{\beta}_{n} \stackrel{\text { a.s. }}{\longrightarrow} \beta-\frac{1}{c}$,

$$
\hat{\alpha}_{n}=\alpha+\left(\beta-\hat{\beta}_{n}\right) \bar{x}_{n}+\bar{\epsilon}_{n} \stackrel{a . s .}{\longrightarrow} \alpha+S .
$$

At last, let us look at what happens to the eigenvalue condition of the design matrix. Let $\mathbf{x}_{i}=\left(1, x_{i}\right)^{\prime}, \mathbf{X}_{n}=\left(\mathbf{x}_{1}, \cdots, \mathbf{x}_{n}\right)^{\prime}$, and $\lambda_{\max }(n)$ and $\lambda_{\min }(n)$ be the maximum and minimum eigenvalue of $\mathbf{X}_{n}^{\prime} \mathbf{X}_{n}$ respectively.

According to Example 1 of Lai and Wei [1982],

$$
\begin{aligned}
& \lambda_{\max }(n) \sim n\left\{1+c^{2}\left(\sum_{i=1}^{\infty} \frac{\epsilon_{i}}{i}\right)^{2}\right\} \text { a.s. } \\
& \lambda_{\min }(n) \sim \frac{c^{2} \log n}{1+c^{2}\left(\sum_{i=1}^{\infty} \frac{\epsilon_{i}}{i}\right)^{2}} \text { a.s., }
\end{aligned}
$$

thus,

$$
\frac{\log \lambda_{\max }(n)}{\lambda_{\min }(n)} \stackrel{\text { a.s. }}{\longrightarrow} \frac{1+c^{2}\left(\sum_{i=1}^{\infty} \frac{\epsilon_{i}}{i}\right)^{2}}{c^{2}}
$$

The limit is not zero, which violates the eigenvalue condition (2.5). 


\section{Chapter 3}

\section{Applications of Repeated Least Squares Procedure}

\subsection{Loss Criteria}

Due to ethical concerns, most dose-finding designs assign the "best" estimated dose to the next patient based on historical data, so as to lower the dosing costs for enrolled subjects as largely as possible. These so-called "best-intent" designs share a common limitation that is overlooking the potential costs of dosing future patients.

To reduce future dosing costs, an individualized dose-finding design cannot do better than estimating the dosing function as precisely as possible. As stated in Section 2.3, design points need to be spread out to ensure the precision of least squares estimator and the strong consistency of dose sequence. Thus, a key to implement RLSEVC is to choose convergence rate of the eigenvalue constraint, which indeed controls the extent of spreading design points apart. A slow-converging constraint would contribute little to spreading design points, and lead RLSEVC to be equivalent to RLS; a fast-converging constraint would force design points to be far away from the "good" doses, and cause large dosing cost. Therefore, the goal of 
design calibration is to identify out an "optimal" pair of $\left(\delta_{1}, \delta_{2}\right)$ for RLSEVC, so that it can balance dosing costs of current subjects and future patients.

Definition 4 (Dosing Cost) In the light of Lai and Robbins [1979], the total dosing cost of patients in a trial is measured by the sum of squared dosing biases:

$$
D_{n}=\sum_{i=1}^{n}\left(x_{i}-\theta\left(z_{i}\right)\right)^{2}
$$

where $n$ is the sample size of a trial.

Definition 5 (Integrated Squared Error) The accuracy of the estimated dosing function $\hat{\theta}_{n+1}(z)$ is assessed by the integrated squared error (ISE):

$$
\mathrm{ISE}=\int_{I_{z}}\left[\hat{\theta}_{n+1}(z)-\theta(z)\right]^{2} p(z) d z,
$$

where $p(z)$ is the normal density of predicted clearance on log-scale $(z)$.

In other words, the dosing cost measures the overall expenses that all $n$ subjects of the current trial would pay for, while ISE indeed measures the potential dosing cost for a single patient who will be dosed using the estimated dosing function in the future.

To account for the two sources of dosing costs, we propose a two-element loss function

$$
L\left(\delta_{1}, \delta_{2}\right)=\bar{D}_{n}+\kappa n \text { MISE, } \quad \text { for some }\left(\delta_{1}, \delta_{2}\right) \in \mathcal{R}
$$

where $\bar{D}_{n}=\frac{1}{N} \sum_{j=1}^{N} D_{n}^{(j)}$ is the average dosing cost over all $N$ simulated trials, MISE $=$ $\frac{1}{N} \sum_{j=1}^{N} \mathrm{ISE}^{(j)}$ is the mean integrated squared error across all simulated trials, and $\kappa(>0)$ is the ratio of a future trial's size compared to the current sample size. Since $\bar{D}_{n}$ measures dosing cost for $n$ current subjects, by timing $\kappa n$ to MISE, we intend to balance the contributions of current and future dosing costs to the loss function, and avoid either of them to dominate the criterion. 
Therefore, the "optimal" design parameter $\left(\delta_{1}, \delta_{2}\right)_{\text {opt }}$ is defined to be the pair that minimizes the loss function (3.3) over $\mathcal{R}$, that is,

$$
\left(\delta_{1}, \delta_{2}\right)_{\text {opt }}=\underset{\left(\delta_{1}, \delta_{2}\right) \in \mathcal{R}}{\arg \min } L\left(\delta_{1}, \delta_{2}\right) .
$$

\subsection{Calibration Scenarios Generation}

The simulations of design calibration are performed under the linear model (2.1) along with the variance $\sigma^{2}$, which are necessary for generating the responses given doses and predicted

clearance values. It would be natural to obtain estimates of the model coefficients and $\sigma^{2}$ by applying least squares fit to the irinorecan data, as in Section 2.1. However, this would cause the calibration results almost depends on a specific true scenario, and largely weaken the generality and reliability of RLSEVC.

Thus, in order to avoid the design calibration to depend on some specific cases, we generate sufficiently many and intensively varying true scenarios, $\left(\alpha, \beta, \gamma, \sigma^{2}\right)$, under a Bayesian framework. Based on the model (2.1), we further assume the following prior distributions on the model coefficients and variance:

$$
\mathbf{b}=(\alpha, \beta, \gamma)^{\prime} \sim N\left(\mathbf{b}_{0}, \tau_{0}^{2} I_{3}\right), \text { and, } \sigma^{2} \sim I G\left(\eta_{0}, \eta_{1}\right),
$$

where the initial mean vector $\mathbf{b}_{\mathbf{0}}$ is chosen as the theoretical coefficients stated by the PK model $1.2, \mathbf{b}_{\mathbf{0}}=(0,1,-1)^{\prime}$, the hyper prior variances of model coefficients are assumed to be common and non-informative as $\tau_{0}^{2}=10^{6}$, and the hyper prior parameters of inverse gamma (IG) prior distribution are also chosen to be non-informative such that $\eta_{0}=\eta_{1}=0.1$.

Thus, given the design matrix $M_{n}$ and response vector $Y_{n}$ of the irinotecan data, the joint 
posterior density function can be derived out as follows

$$
\begin{aligned}
& p\left(\mathbf{b}, \sigma^{2} \mid M_{n}, Y_{n}\right) \propto\left\{\prod_{i=1}^{n} f\left(y_{i} \mid \mathbf{b}, \sigma^{2}, m_{i}\right)\right\} f(\mathbf{b}) f\left(\sigma^{2}\right) \\
& \propto \exp \left\{-\frac{1}{2 \sigma^{2}} \sum_{i=1}^{n}\left(y_{i}-\mathbf{b}^{\prime} m_{i}\right)^{2}-\frac{1}{2 \tau_{0}^{2}}\left(\mathbf{b}-\mathbf{b}_{\mathbf{0}}\right)^{\prime}\left(\mathbf{b}-\mathbf{b}_{\mathbf{0}}\right)\right\}\left(\sigma^{2}\right)^{-n / 2-\eta_{0}-1} \exp \left\{-\frac{\eta_{1}}{\sigma^{2}}\right\} \\
& =\exp \left\{-\frac{1}{2 \sigma^{2}}\left(Y_{n}-M_{n} \mathbf{b}\right)^{\prime}\left(Y_{n}-M_{n} \mathbf{b}\right)-\frac{1}{2 \tau_{0}^{2}}\left(\mathbf{b}-\mathbf{b}_{\mathbf{0}}\right)^{\prime}\left(\mathbf{b}-\mathbf{b}_{\mathbf{0}}\right)-\frac{\eta_{1}}{\sigma^{2}}\right\}\left(\sigma^{2}\right)^{-n / 2-\eta_{0}-1}
\end{aligned}
$$

where $M_{n}=\left(m_{1}, \cdots, m_{n}\right)^{\prime}$ and $m_{i}=\left(1, x_{i}, z_{i}\right)^{\prime}$ for $i=1, \cdots, n$.

Proposition 1 (Marginal Posterior Distributions) Given the joint posterior density (3.4), there can derived out the following marginal posterior distributions respectively:

$$
\begin{aligned}
& p\left(\mathbf{b} \mid Y_{n}, M_{n}, \sigma^{2}\right) \propto N\left(\Sigma_{n} \Psi_{n}, \sigma^{2} \Sigma_{n}\right), \text { and, } \\
& p\left(\sigma^{2} \mid Y_{n}, M_{n}, \mathbf{b}\right) \propto I G\left(\eta_{0}+\frac{n}{2}, \eta_{1}+\frac{\Delta_{n}}{2}\right),
\end{aligned}
$$

where $I_{3}$ is the three dimensional elementary matrix, $\Sigma_{n}=\left(M_{n}^{\prime} M_{n}+\frac{\sigma^{2}}{\tau_{0}^{2}} I_{3}\right)^{-1}, \Psi_{n}=$ $M_{n}^{\prime} Y_{n}+\frac{\sigma^{2}}{\tau_{0}^{2}} \mathbf{b}_{\mathbf{0}}$, and $\Delta_{n}=\left(Y_{n}-M_{n} \mathbf{b}^{\prime}\right)^{\prime}\left(Y_{n}-M_{n} \mathbf{b}^{\prime}\right)$.

Proof:

$$
\begin{aligned}
& p\left(\mathbf{b} \mid Y_{n}, M_{n}, \sigma^{2}\right) \propto\left\{\prod_{i=1}^{n} f\left(y_{i} \mid \mathbf{b}, \sigma^{2}, m_{i}\right)\right\} f(\mathbf{b}) \\
& \propto \exp \left\{-\frac{1}{2 \sigma^{2}}\left(Y_{n}-M_{n} \mathbf{b}\right)^{\prime}\left(Y_{n}-M_{n} \mathbf{b}\right)-\frac{1}{2 \tau_{0}^{2}}\left(\mathbf{b}-\mathbf{b}_{\mathbf{0}}\right)^{\prime}\left(\mathbf{b}-\mathbf{b}_{\mathbf{0}}\right)\right\} \\
& =\exp \left\{-\frac{1}{2 \sigma^{2}}\left(Y_{n}^{\prime} Y_{n}-2 Y_{n}^{\prime} M_{n} \mathbf{b}+\mathbf{b}^{\prime} M_{n}^{\prime} M_{n} \mathbf{b}\right)-\frac{1}{2 \tau_{0}^{2}}\left(\mathbf{b}^{\prime} \mathbf{b}-2 \mathbf{b}_{\mathbf{0}}{ }^{\prime} \mathbf{b}+\mathbf{b}_{\mathbf{0}}{ }^{\prime} \mathbf{b}_{\mathbf{0}}\right)\right\} \\
& \propto \exp \left\{-\frac{1}{2 \sigma^{2}}\left[\mathbf{b}^{\prime}\left(M_{n}^{\prime} M_{n}+\frac{\sigma^{2}}{\tau_{0}^{2}} I_{3}\right) \mathbf{b}-2\left(Y_{n}^{\prime} M_{n}+\frac{\sigma^{2}}{\tau_{0}^{2}} \mathbf{b}_{\mathbf{0}}{ }^{\prime} I_{3}\right) \mathbf{b}\right]\right\} \\
& \propto \exp \left\{-\frac{1}{2 \sigma^{2}}\left(\mathbf{b}^{\prime} \Sigma_{n}^{-1} \mathbf{b}-2 \Psi_{n}^{\prime} \Sigma_{n} \Sigma_{n}^{-1} \mathbf{b}+\Psi_{n}^{\prime} \Sigma_{n} \Sigma_{n}^{-1} \Sigma_{n} \Psi_{n}\right)\right\}
\end{aligned}
$$




$$
\begin{aligned}
& =\exp \left\{-\frac{1}{2 \sigma^{2}}\left(\mathbf{b}-\Sigma_{n} \Psi_{n}\right)^{\prime} \Sigma_{n}^{-1}\left(\mathbf{b}-\Sigma_{n} \Psi_{n}\right)\right\} \\
& \propto N\left(\Sigma_{n} \Psi_{n}, \sigma^{2} \Sigma_{n}\right) ; \\
& p\left(\sigma^{2} \mid Y_{n}, M_{n}, \mathbf{b}\right) \propto\left\{\prod_{i=1}^{n} f\left(y_{i} \mid \mathbf{b}, \sigma^{2}, m_{i}\right)\right\} f\left(\sigma^{2}\right) \\
& \propto \exp \left\{-\frac{1}{2 \sigma^{2}}\left(Y_{n}-M_{n} \mathbf{b}\right)^{\prime}\left(Y_{n}-M_{n} \mathbf{b}\right)-\frac{\eta_{1}}{\sigma^{2}}\right\}\left(\sigma^{2}\right)^{-n / 2-\eta_{0}-1} \\
& =\left(\sigma^{2}\right)^{-\left(n / 2+\eta_{0}\right)-1} \exp \left\{-\frac{1}{\sigma^{2}}\left(\eta_{1}+\Delta_{n} / 2\right)\right\} \\
& \propto I G\left(\eta_{0}+\frac{n}{2}, \eta_{1}+\frac{\Delta_{n}}{2}\right) .
\end{aligned}
$$

Based on the marginal posterior densities (3.5), given the irinotecan data and hyper prior parameters, we at first apply the Gibbs sampling algorithm to generate in total 100,000 pairs of $\left(\alpha, \beta, \gamma, \sigma^{2}\right)$ from these posterior distributions. Actually, in order to ensure the generated model coefficients satisfying that $\beta>0, \gamma \leq 0$, and $\theta(z) \in I_{x}$, the Gibbs sampling is carried out by keeping only the draws that meet these restrictions until the total number reaches 100,000. At the second, provided that this generated Monte Carlo Markov Chain (MCMC) converges very quickly, the first 1000 pairs of the draws are burned out. At last, $N=10000$ pairs are randomly sampled from the rest draws so as to reduce the auto-correlations among the MCMC samples downto a acceptably low level.

These final 10000 pairs, denoted as $\left(\alpha^{(j)}, \beta^{(j)}, \gamma^{(j)}, \sigma_{j}^{2}\right)$ for $j=1, \cdots, N$, will be used as the true models to generate the response in the simulations of design calibration. In this way, sufficiently many true scenarios are generated via deeply digging information from the irinotecan data. 


\subsection{Calibrating the Eigenvalue Constraint}

In the numerical simulations, in total 166 candidate pairs of $\left(\delta_{1}, \delta_{2}\right)$ are evenly selected within the area $[0,0.6] \times[0,6] \backslash 0 \times[0,1]$. Specifically, $\delta_{1}$ ranges from 0 to 0.6 with a step size of 0.05, meanwhile $\delta_{2}$ varies from 0 to 6 with a step size of 0.5 , only excluding the cases that $\delta_{2} \in\{0,0.5,1\}$ when $\delta_{1}=0$. The actual selection area of $\left(\delta_{1}, \delta_{2}\right)$ is chosen not as large as the theoretical area $\mathcal{R}$, because the eigenvalue constraint would be too restricted to satisfy if either $\delta_{1}$ or $\delta_{2}$ is too large.

It is worth to mention that, prior to either RLS or RLSEVC, an initial design (size of $\left.n_{0}\right)$ is necessary for enabling the least squares estimation of the model (2.1). Since there are 3 unknown coefficients $(\alpha, \beta, \gamma)$ in the model $(2.1)$, at least 3 observations are needed to initiate the least squares fit. Throughout the simulations of this dissertations, we apply the BSA formula (1.4) to calculate doses in the initial trial, meanwhile determine its sample size $n_{0}$ in a dynamic way. In the numerical simulations, $n_{0}$ is most often not larger than 5 .

Specifically, the sample size of initial trial begins with $n_{0}=3$; if $\left|M_{n_{0}}^{\prime} M_{n_{0}}\right| \geq \delta_{\text {det }}$, then the trial is switched from the initial design to RLSEVC; if $\left|M_{n_{0}}^{\prime} M_{n_{0}}\right|<\delta_{\text {det }}$, then one more subject is recruited into the initial trial (sample size increased to $n_{0}+1$ ), and the determinant is re-evaluated; this procedure will be continued until the determinant exceeds $\delta_{\text {det }}$, where $\delta_{d e t}$ is a pre-selected small threshold and $M_{n_{0}}$ is the initial design matrix. This dynamic sample size determination procedure is to avoid that the singularity of initial design matrix causes poor initial least squares estimation.

Moreover, another important benefit of the initial design is that, we can utilize the

eigenvalue ratio $\rho_{n_{0}}=\frac{\log \lambda_{\max }\left(n_{0}\right)}{\lambda_{\min }\left(n_{0}\right)}$ to estimate the constant $C$ in the eigenvalue constraint (2.6), where $\lambda_{\max }\left(n_{0}\right)$ and $\lambda_{\min }\left(n_{0}\right)$ are respectively the maximum and minimum eigenvalues of $M_{n_{0}}^{\prime} M_{n_{0}}$. In this way, the choice of $C$ will be data-driven, and avoid subjective selection.

\section{Algorithm 3 (Calibration of RLSEVC)}

For each candidate pair of $\left(\delta_{1}, \delta_{2}\right)$, under the $j$ th true scenario $\left(\alpha^{(j)}, \beta^{(j)}, \gamma^{(j)}, \sigma_{j}^{2}\right)$, a trial 
of size $n=40$ is simulated using RLSEVC design. For the $(i+1)$ th subject, $i=n_{0}, \cdots, n$, given $\left(\hat{\alpha}_{i}^{(j)}, \hat{\beta}_{i}^{(j)}, \hat{\gamma}_{i}^{(j)}\right)$,

1. Estimate the dosing function by $\hat{\theta}_{i}^{(j)}(z)=\frac{t_{0}-\hat{\alpha}_{i}^{(j)}-\hat{\gamma}_{i}^{(j)} z}{\hat{\beta}_{i}^{(j)}}$ for any fixed $z \in I_{z}$;

2. Search for $x_{i+1}=\underset{x \in I_{X}}{\arg \min }\left|x-\hat{\theta}_{i}^{(j)}\left(z_{i+1}\right)\right|$ subject to the eigenvalue constraint $\rho_{i+1}\left(x, z_{i+1}\right) \leq r_{i+1}$, where $r_{i+1}=C(i+1)^{-\delta_{1}}(\log (i+1))^{1-\delta_{2}} ;$

3. Simulate $y_{i+1}=\alpha^{(j)}+\beta^{(j)} x_{i+1}+\gamma^{(j)} z_{i+1}+\epsilon_{i+1}$, where $\epsilon_{i+1} \sim N\left(0, \sigma_{j}^{2}\right)$, and then update the least squares estimates $\left(\hat{\alpha}_{i+1}^{(j)}, \hat{\beta}_{i+1}^{(j)}, \hat{\gamma}_{i+1}^{(j)}\right)$.

4. Repeat Step 1 through 3 until $i$ reaches $n=40$; at the end of this trial, the dosing cost $D_{n}^{(j)}$ and $\operatorname{ISE}^{(j)}$ will be computed.

For each trial, the $n$ subjects are simulated via generating $n$ pairs of $\left(z_{i}, B_{i}, \epsilon_{i}\right)$. Specifically, $\epsilon_{i}$ 's are iid samples from the univariate normal distribution $N\left(0, \sigma_{j}^{2}\right)$; meanwhile, $\left(z_{i}, B_{i}\right)$ is generated jointly from a bi-variate normal distribution, whose mean vector and covariance matrix are respectively estimated by the corresponding sample estimates based on the irinotecan data (See Table 1.2 in Section 1.4.2).

Throughout the simulations of this dissertation, besides the design calibration, we make adequate use of the irinotecan data. For example, according to the sample ranges for irinotecan dose and predicted clearance on log-scale, the ranges of $x$ and $z$ are selected to be $I_{x}=[5,8]$ and $I_{z}=[1.9,5]$. They are set slightly wider than their corresponding confidence intervals with radius of 6 standard deviations. Particularly, if the dose range is too narrow, it would help RLS correct the estimated dosing function by truncating it with over-informative upper and lower limits. Also, a very narrow range of predicted clearance would make the improvement in MISE between RLS and RLSEVC appear not to be as large as the real difference. Thus, we select the ranges to be slightly wider than that obtained from irinotecan data, in order to avoid informing the designs too much about the true dosing function, and 


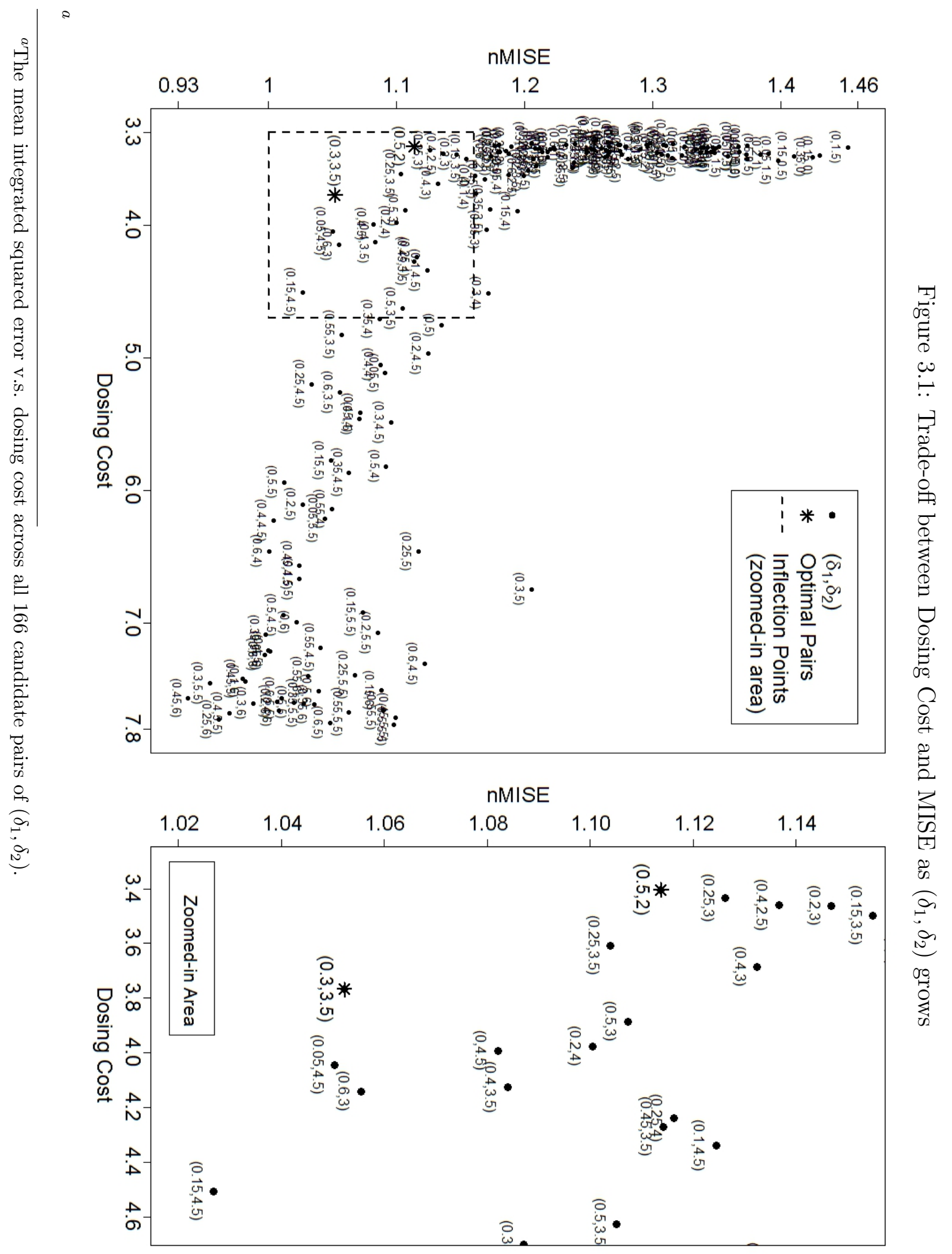


maintain a comparatively large domain space to highlight the difference between estimated dosing functions.

Figure 3.1 presents a trade-off trend between MISE and dosing cost across all candidate pairs of $\left(\delta_{1}, \delta_{2}\right)$. As the magnitude of $\left(\delta_{1}, \delta_{2}\right)$ increases, MISE is gradually improved, while the dosing cost stays constantly around the lowest level for small pairs and then grows dramatically since some "inflection" points among intermediate pairs. The trade-off pattern demonstrates that, spreading design points can improve the least squares estimation, but would sacrifice certain dosing cost. The optimal pairs of $\left(\delta_{1}, \delta_{2}\right)$ that minimizes the loss function (3.3) are, respectively, $(0.5,2)$ for $1 \leq \kappa \leq 5$, and $(0.3,3.5)$ for $5<\kappa \leq 25$. The two ranges of $\kappa$ respectively represent small and medium-to-large patient horizons of a future trial. Both the two optimal pairs (highlighted as stars in Figure 3.1) are typical representatives of these "inflection" points. For a small patient horizon $(\kappa \leq 5)$, the dosing cost of RLSEVC with the optimal pair $(0.5,2)$ nearly remains the lowest meanwhile its MISE is improved significantly; for comparatively larger $\kappa$, RLSEVC with $(0.3,3.5)$ trades mild dosing cost with additional improvement in MISE.

The left two plots of Figure 3.2 show the tendency of dosing cost and MISE depending on $\delta_{1}$ for each fixed $\delta_{2}$; and, the right two show the $\delta_{1}$-specific dependency of dosing cost and MISE on $\delta_{2}$. Generally speaking, $\delta_{2}$ has larger impact on the two quantities than $\delta_{1}$ does. For specific $\delta_{2}$ ranging from 0 to 2.5, the dosing cost is not influenced by $\delta_{1}$, while for $\delta_{2}$ larger than 3 , the dosing cost grows quickly as $\delta_{1}$ increases. For every given $\delta_{1}$, the dosing cost similarly maintains at a low level for $\delta_{2}$ smaller than 2.5, and then dramatically grows as $\delta_{2}$ increases beyond 2.5. MISE is generally not affected by the variation of $\delta_{1}$, and decreases significantly as $\delta_{2}$ grows.

Figure 3.3 presents $\delta_{1^{-}}$and $\delta_{2^{-}}$specific dependency of the loss on the other one, given two representative patient horizons. For $\kappa=3$, the patterns of loss are more similar to those of overall dosing cost, and thus the resultant optimal pair of $\left(\delta_{1}, \delta_{2}\right)$ emphasizes the dosing cost 

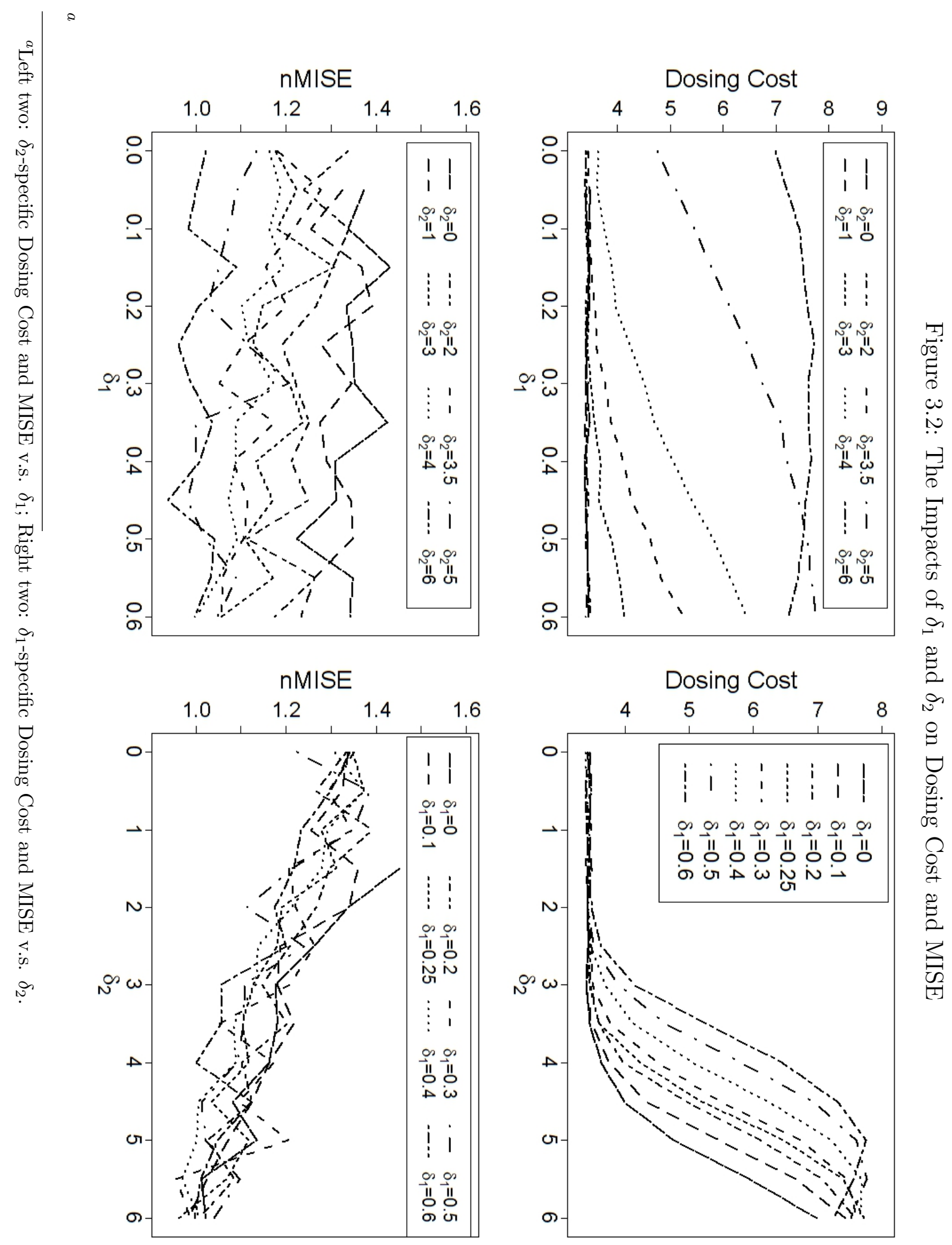


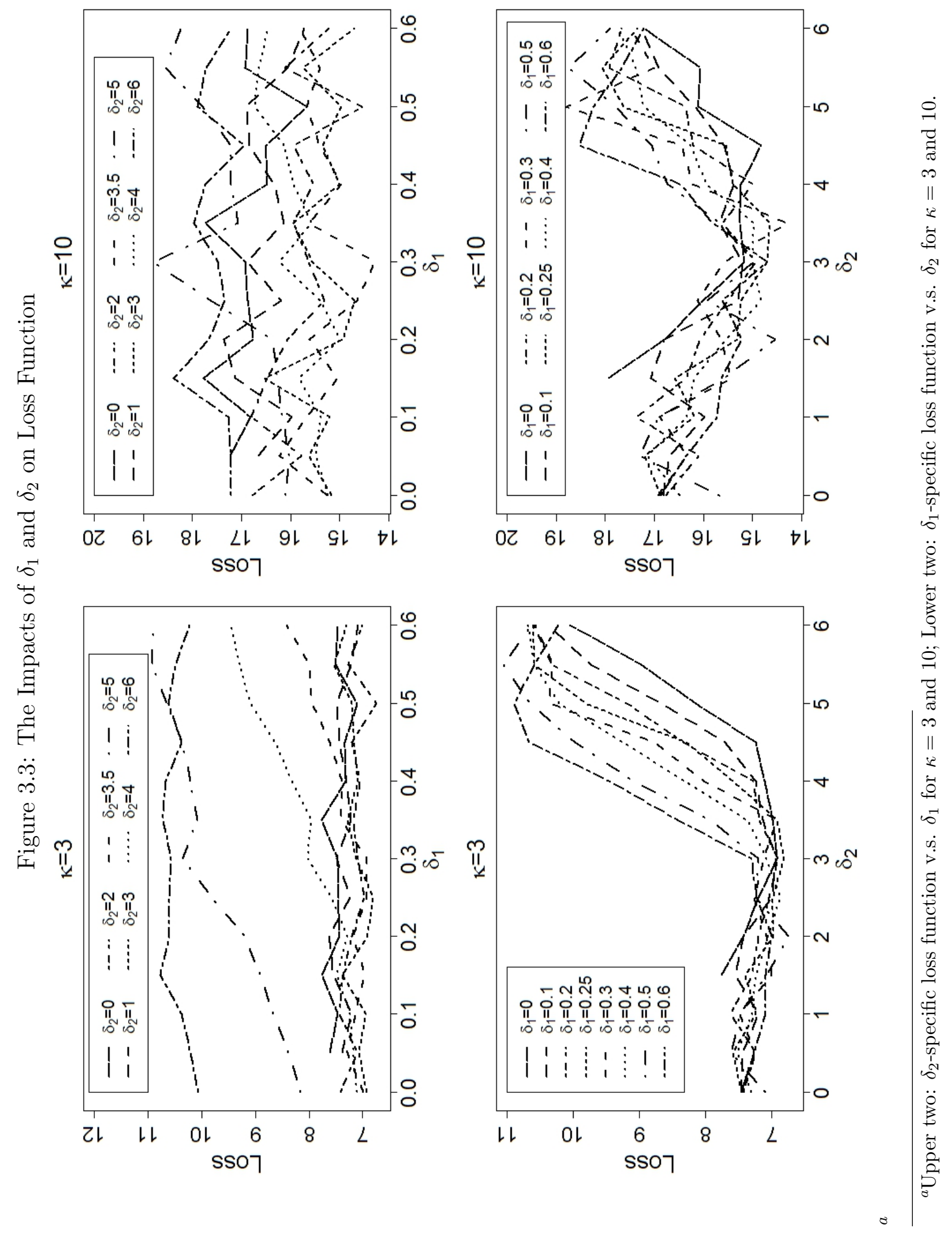


of current trial more than that of future patients. For $\kappa=10$, MISE contributes more to the loss, which leads the corresponding optimal pair to emphasize more on the dosing cost for future patients. The choice of $\kappa$ actually relies on the size of a future trial compared to that of the current, and hence is not possible to explicitly enumerated and evaluated here.

For the loss function, the choice of $\kappa$ is subtle, as it relies on the size of a future trial. If a future trial has a small size $(1 \leq \kappa \leq 5)$, then a less restricted constraint $(0.5,2)$ would be preferred to emphasize on controlling current dosing cost. If the size of a future trial is much bigger than the current $(5<\kappa \leq 25)$, then a more restricted constraint $(0.3,3.5)$ should be advocated to reduce as much as possible the potential dosing cost paid by future patients. After all, for the welfare of a large number of future patients, it is worth to sacrifice some minor welfare of current subjects, as long as which is controlled within an acceptable range.

Consequently, by imposing the eigenvalue constraint on RLS, not only MISE can be constantly improved as the constraint tightens gradually, but can the dosing cost also remain at nearly the lowest level for some "inflection" pairs of $\left(\delta_{1}, \delta_{2}\right)$. These "inflection" pairs of $\left(\delta_{1}, \delta_{2}\right)$ could be options of the optimal choice. Our proposed loss criterion succeeds in identifying the "optimal" pairs of $\left(\delta_{1}, \delta_{2}\right)$ according to different patient horizons. By equipping with the "optimal" pairs of $\left(\delta_{1}, \delta_{2}\right)$, RLSEVC is able to improve precision of least squares estimation significantly, the same time sacrifice little overall dosing cost.

\subsection{An Illustrative Trial}

In this section, we demonstrate the effects of eigenvalue constraint and coherence restriction by simulating an illustrative trial. Specifically, for the same 40 subjects (in the sense of common $\left.\left(z_{i}, B_{i}, \epsilon_{i}\right), i=1, \cdots, n\right)$, we separately simulate a trial (see Figure 3.4) utilizing each of the BSA, equation, RLS, RLSEVC, RLS-CR and RLSEVC-CR designs, where RLSCR denotes RLS with coherence restriction and RLSEVC-CR likewise. 

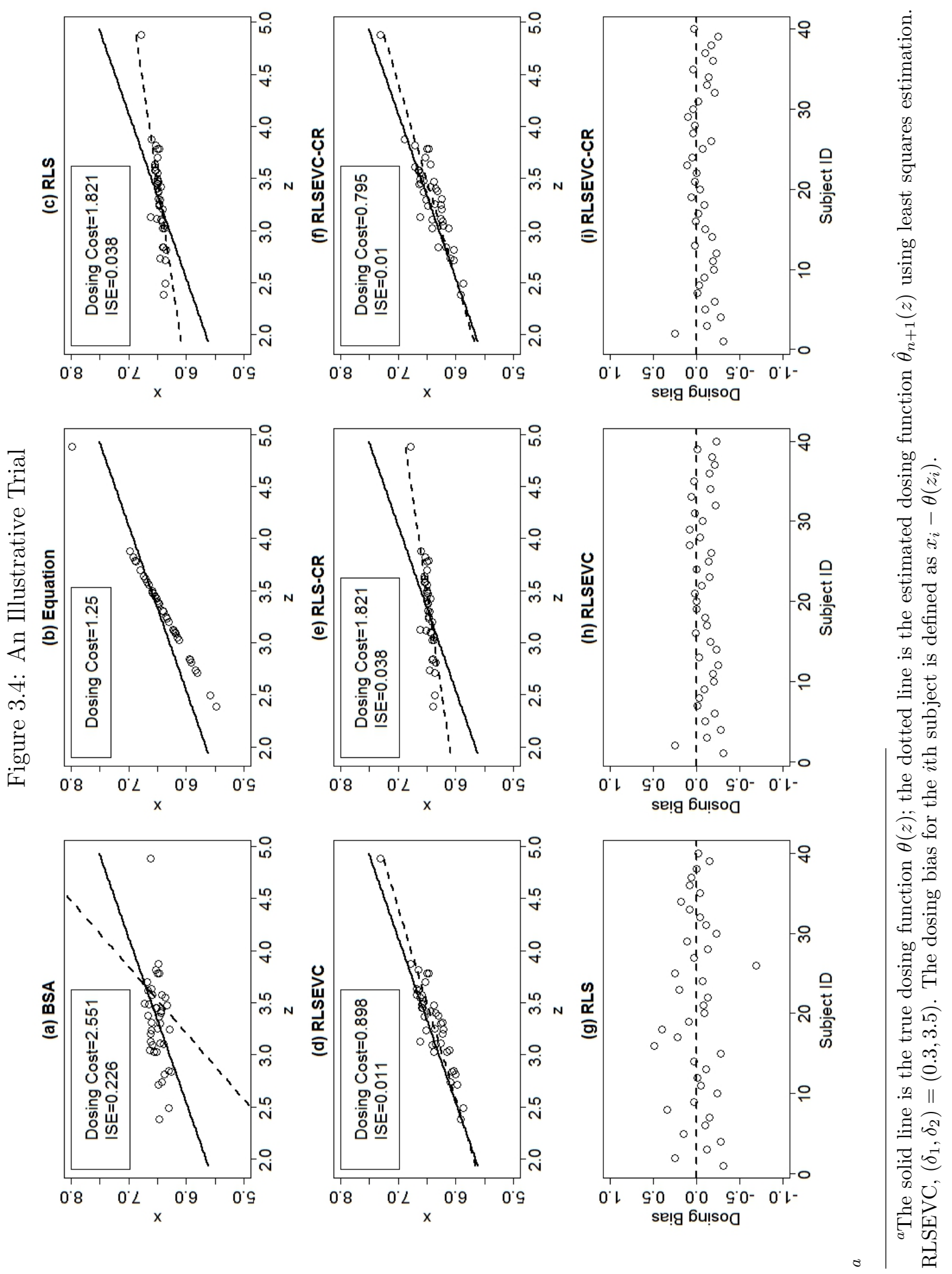
The effect of eigenvalue constraint on excluding the asymptotic singularity and improving precision of least squares estimator can be found by comparing (c) with (d) of Figure 3.4. Most doses suggested by RLS (c) are stuck at a wrong dosing function that is substantially biased from the true one, in that the dose sequence converges fast to the wrong target without receiving any correction from the design. This results in poorly estimated dosing function $(\mathrm{ISE}=0.038)$ at the trial end and large dosing cost (1.821) to the trial. In contrast, RLSEVC (d) succeeds in forcing design points to slightly spread out along the true dosing function, as the eigenvalue constraint gradually becomes effective. As a result, RLSEVC largely corrects the estimated dosing function towards the true one (ISE $=0.011)$ meanwhile reduce the dosing cost to 0.898. Moreover, by comparing (g) and (h), it can be found that, except for the subjects of initial trial $\left(n_{0}=4\right)$, the RLSEVC trial almost constantly has smaller dosing biases than the RLS does. It reflects the consistency of dose sequence recommended by RLSEVC.

Both the BSA (a) and equation (b) based methods fail to provide correct information to estimate the true dosing function. BSA randomly "guesses" dosage for a subject according to his/her body surface area, which does not have high association with the predicted clearance. The equation method could result in large dosing cost, unless the equation is known to be very close to the true dosing function, which is not realistic in practice. Moreover, the equation method provides no information for least squares estimation, since the dose column is co-linear with the other columns in its design matrix.

The effect of coherence restriction on further reducing dosing cost for RLSEVC can be found by comparing (d) and (f) of Figure 3.4. When the coherence restriction is applied to RLSEVC, the dosing cost is lowered from 0.898 to 0.795 , while the ISE is also reduced slightly. Comparison between (h) and (i) of Figure 3.4 also shows that more subjects are benefited with smaller dosing biases in the presence of coherence restriction. It is worth to mention that, when the asymptotic singularity is present, the coherence restriction cannot 
help RLS with reducing dosing cost or ISE at all (see Figure 3.4 (c) and (e)), which two trials are exactly the same.

\subsection{Design Comparison under Linear Models}

In this section, we present a simulation study comparing performance across the competing designs, BSA, Equation, RLS and RLSEVC under linear models, in terms of the dosing cost and MISE. By setting $\left(\delta_{1}, \delta_{2}\right)=(-\infty,-\infty)$, RLS actually becomes a special case of RLSEVC, where the eigenvalue constraint has no effect. For RLSEVC, $\left(\delta_{1}, \delta_{2}\right)$ is chosen at the "optimal" pairs, $(0.5,2)$ and $(0.3,3.5)$. In addition, to highlight the effect of coherence restriction, we also particularly include RLS and RLSEVC with and without the coherence restriction as competing designs in the comparison.

The simulations of design comparison are performed under six representative scenarios (see Figure 3.5), which are carefully selected to assess the robustness of these designs.

Table 3.1: True Scenarios in Design Comparison under Linear Model

\begin{tabular}{cccccc}
\hline \hline Scenario $^{a}$ & $\alpha$ & $\beta$ & $\gamma$ & $\frac{t_{0}-\alpha}{\beta}$ & $-\frac{\gamma}{\beta}$ \\
\hline 1 & 0.1236 & 0.6768 & -0.4275 & 4.3950 & 0.6316 \\
2 & -0.5566 & 0.6768 & -0.4275 & 5.4000 & 0.6316 \\
3 & 1.7500 & 0.6768 & -0.8200 & 1.9920 & 1.2116 \\
4 & -1.6395 & 0.6768 & 0.0000 & 7.0000 & 0.0000 \\
5 & -0.2182 & 0.6768 & -0.2166 & 4.9000 & 0.3200 \\
6 & 3.0982 & 0.6768 & -1.3536 & 0.0000 & 2.0000 \\
\hline
\end{tabular}

${ }^{a} \sigma=0.2618$ is common for all the 6 scenarios.

Among the scenarios, the 1st is typically generated by the Gibbs sampling under the Bayesian framework as introduced in Section 3.3. It is "regular" in the sense of $(\alpha, \beta, \gamma)^{\prime}$ satisfying that $\theta(z)=\left(t_{0}-\alpha-\gamma z\right) / \beta \in I_{x}$ for any fixed $z \in I_{z}$. Based on the 1st scenario, we create the others by changing either the intercept $\left(t_{0}-\alpha\right) / \beta$ or slope $-\gamma / \beta$ to some extreme cases, in order to account for extreme dosing functions that are not covered by 
those generated using the irinotecan data under the Bayesian framework. For instance, the 2nd, 3rd and 6th scenarios are "irregular" because their true dosing functions exceed the dose range $I_{x}$ on at least one side of the function. Therefore, the true dosing functions of these scenarios are truncated by upper and lower limits of $I_{x}$ where they fall out of the boundaries. Under each scenario, $N=10000$ times of simulations are replicated, and the results are summarized in Table 3.2 .

Figure 3.5: True Dosing Functions of Design Comparison under Linear Model

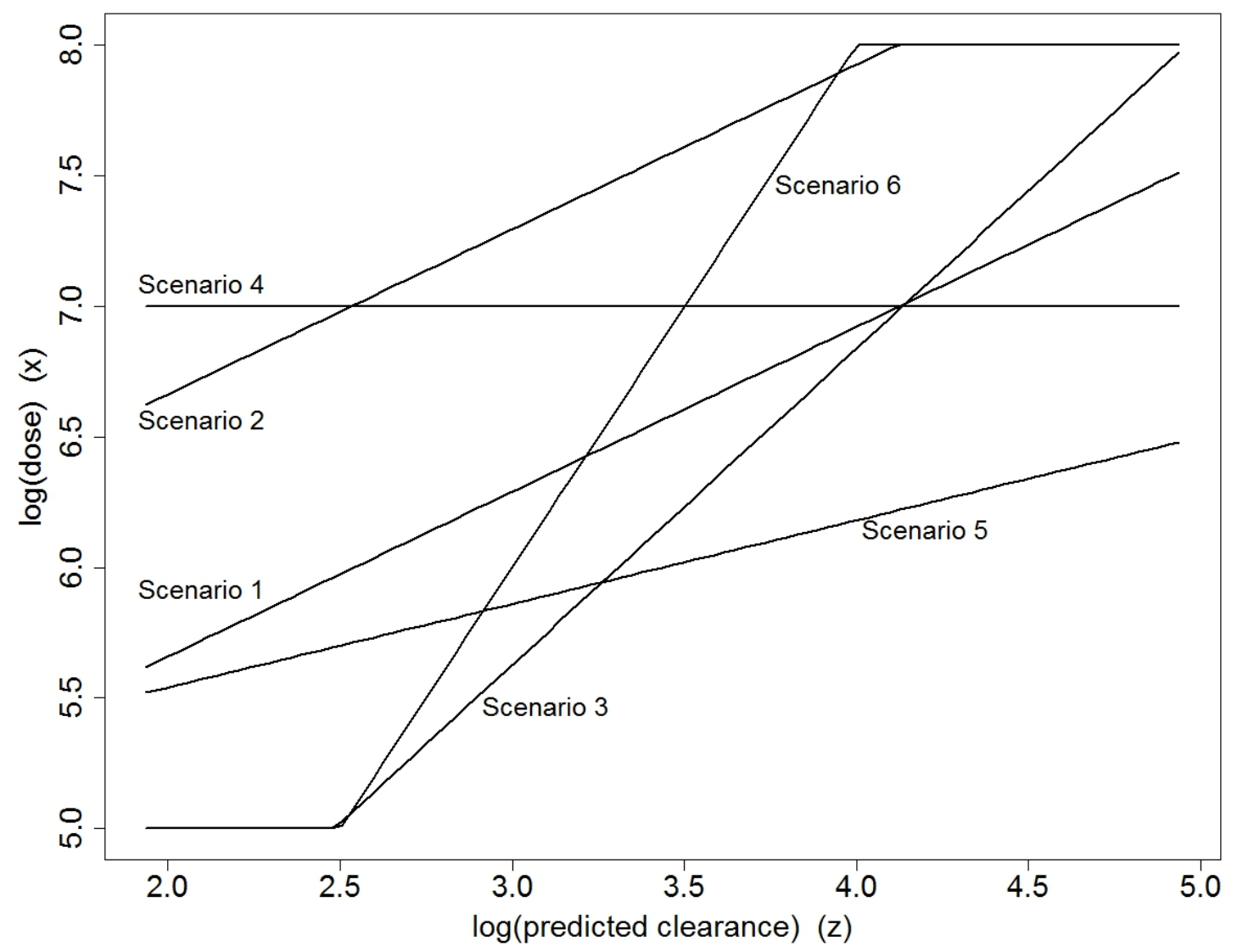

In table (3.2), the average of dosing cost (3.1) and MISE (3.2) over all simulated trials are respectively summarized for each design under each scenario, where the loss function (3.3) is computed with $\kappa=10$. All these numbers are the smaller the better, while MISE is multiplied by 100 to save displaying digits and make the numbers appear reader-friendly.

Generally speaking, across all scenarios, the BSA and equation designs perform constantly 


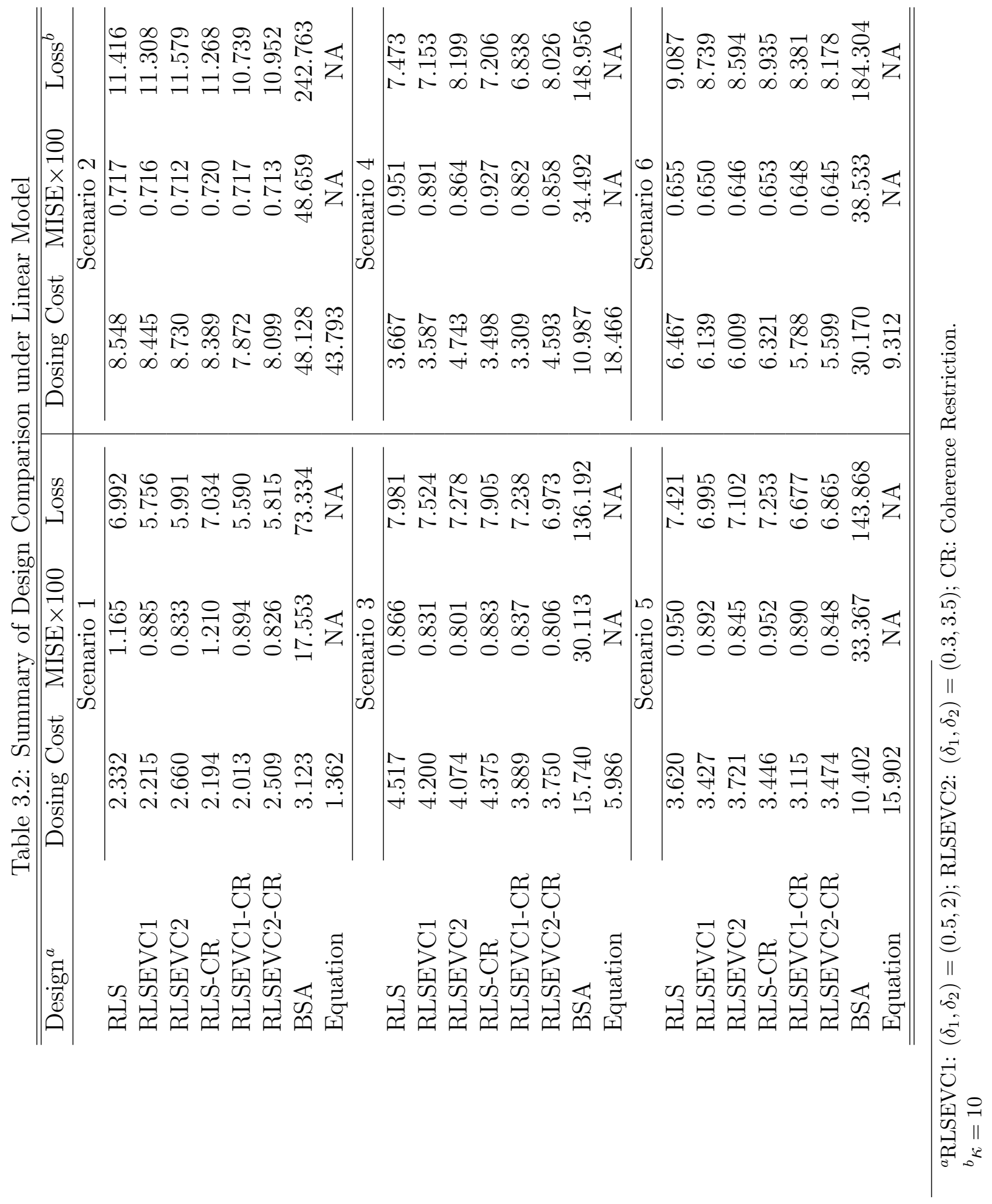


worse than RLS and RLSEVC, in terms of both the dosing cost and MISE. Their performance largely depend on the true scenario, because their dosing costs are out of control at all scenarios other than the 1st. The BSA algorithm fails to provide useful design information to estimate the dosing function and thus result in the worst MISE across all scenarios; the equation method is not able to estimate dosing function using least squares, hence its MISE is not available all the time. In clinical practices, where the true dosing function is never known, applying such unstable designs as the BSA or equation algorithm would expose patients to severe safety issues.

In contrast, RLS and RLSEVC improve MISE significantly meanwhile control the dosing cost robustly, even under the extreme scenarios. Although the performance of RLS seems acceptable in general, due to failing to exclude the asymptotic singularity, its MISE is constantly worse than that of RLSEVC. Because the asymptotic singularity is a phenomenon that does not happen frequently, the improvement in MISE from RLS to RLSEVC does not seem as significant as that from BSA to them. However, it is the ability of overcoming the asymptotic singularity that gives RLSEVC the reliability and safety that RLS is in short of, because "exceptions" are not acceptable in real practices.

For regular scenarios (the 1st, 4th and 5th), the eigenvalue constraint helps RLSEVC significantly improve MISE over RLS, for instance 24 percent reduction (from 1.16 to 0.88 ) in MISE at Scenario 1; moreover, when $\left(\delta_{1}, \delta_{2}\right)_{o p t}=(0.5,2)$, RLSEVC can also reduce the dosing cost compared to RLS. For irregular scenarios (the 2nd, 3rd and 6th), the truncation by the dose range $I_{x}$ largely helps RLS improve estimation accuracy, and hence result in similar MISE with RLSEVC at the 2nd and 6th scenarios, while RLSEVC still largely reduces MISE compared to RLS at the 3rd scenario. However, RLSEVC constantly achieves smaller dosing costs than RLS does at these irregular cases. The trade-off between the overall dosing cost and MISE is still clear for RLSEVC across all scenarios. Thus, the choice between the pairs of $\left(\delta_{1}, \delta_{2}\right)_{\text {opt }}$ is subtle indeed, and should depend on the size of a future trial as we 
discussed in Section 3.3.

By respectively comparing RLS-CR and RLSEVC-CR with those without the coherence restriction, we can find that the coherence restriction further lowers the dosing cost effectively, with maintain the same level of MISE (for instance scenario 1) or even gaining mild improvement in MISE (for instance scenario 4 and 5). Another advantage of the coherence restriction is that, it can also shrink the searching range for the dose that minimizes the loss function, and thus substantially save computation time for implementing RLSEVC.

At last, the dosing cost per each subject across an RLSEVC trial is also recorded, in order to investigate the overall trend in which the individual dosing cost varies as a trial proceeds. Specifically, the individual dosing cost is defined as $\left(x_{i}-\theta\left(z_{i}\right)\right)^{2}$ for $i=1, \cdots, n$. In Figure 3.6, across RLS and RLSEVC with coherence restriction as well as the BSA and equation designs, the average individual dosing cost over all simulated trials is plotted against the subject sequence number under all the 6 scenarios.

From Figure 3.6, it can be clearly told that the individual dosing costs of either BSA or equation algorithm do not vary with the trial process at all. So, in these two designs, the "costs" paid by subjects enter a trial earlier are not used to benefit the later subjects, which is indeed a waste of valuable information resources. Moreover, for BSA and equation algorithms, the dependence of individual dosing cost on the true scenario is obvious. For instance, the cost can be fairly small when using the equation algorithm under Scenario 1, because where the true dosing function is close to that specified by the equation algorithm (1.3); but, the cost becomes very large for the equation algorithm under Scenario 4, as its fixed dosing formula (1.3) now is biased away from the true dosing function too much. As mentioned previously, the true scenario is never known in reality, hence the practice using such unreliable dosing algorithms is highly risky for patients.

For RLS and RLSEVC, a clear pattern that the individual dosing cost increases slightly at beginning, then dramatically decreases since around the 10th subject, and downward 

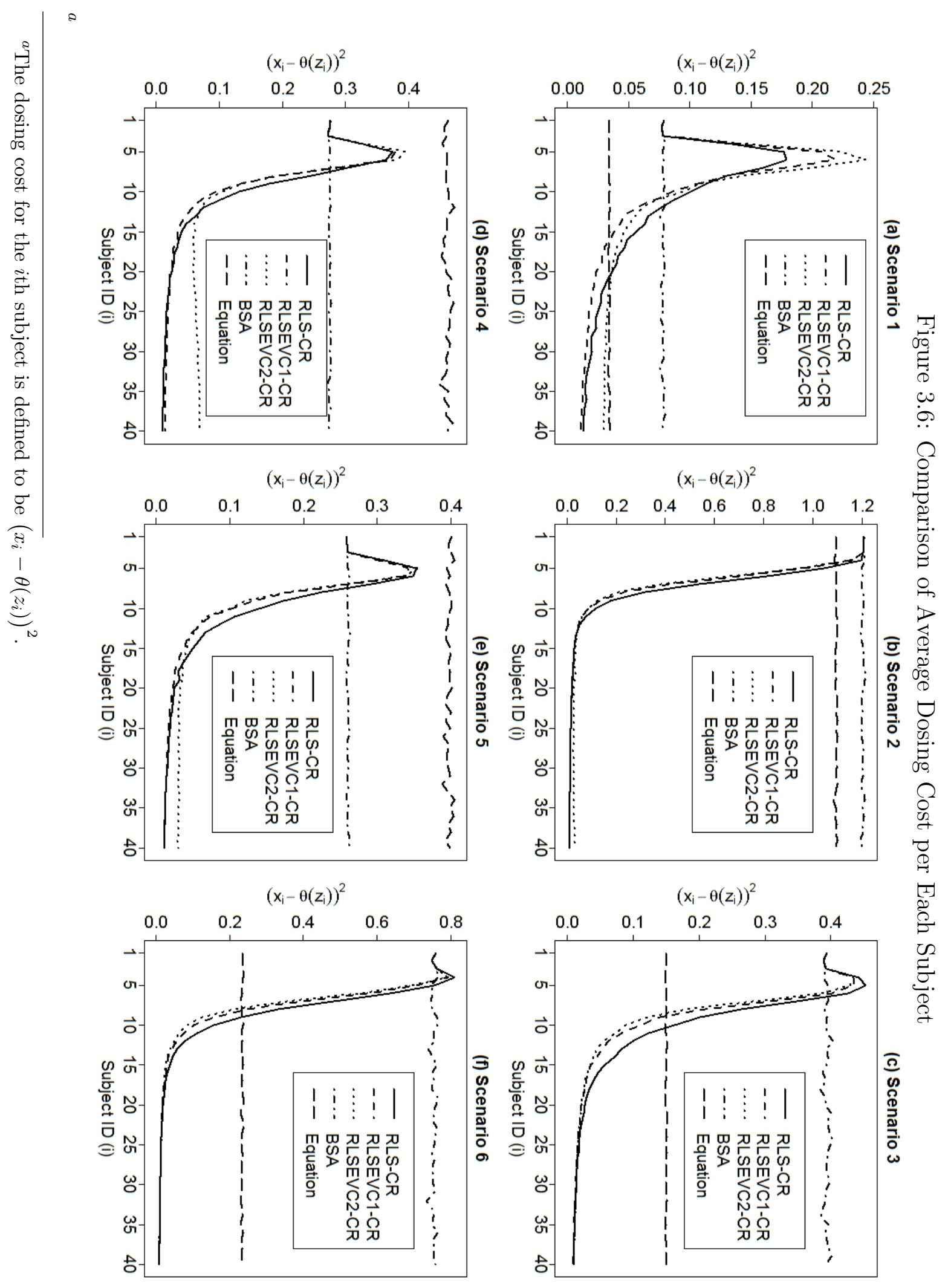
approach zero in the later half trial is shown under all scenarios in Figure 3.6. This pattern not only indicates that the costs paid by early subjects are well utilized to optimizing dose assignments to later subjects, but also demonstrates that the dose sequence recommended by RLSEVC is consistent. The reason why RLS almost behaves as well as RLSEVC does in terms of the individual dosing cost is still that, the asymptotic singularity does not occur frequently and so leads to slight difference on the overall average costs. Since the initial design is based on the BSA formula, the cost of either RLS or RLSEVC is always the same as that of BSA algorithm for the first 3 subjects. If the starting points are close to the true dosing function, such as Scenario 1, the eigenvalue constraint would force the upcoming several design points to spread out certainly, which explains the increment in dosing cost at beginning; upon the design collects sufficient information to ensure estimation precision, the costs quickly decrease to zero in later half trial. In other words, the first several subjects would pay costs for the benefits of most later subjects among an RLSEVC trial.

Consequently, we strongly advocate the use of RLSEVC with coherence restriction, since it not only controls the dosing cost well, estimates the dosing function precisely, but is also robust to extreme dosing scenarios. 


\section{Chapter 4}

\section{Semiparametric Link-Adapted}

\section{Recursion}

\subsection{Semiparametric Dose-Response Model}

The most fundamental assumption that the repeated least squares procedure relies on is the linear relationship (2.1) between the AUC, dose and predicted clearance on log-scale. However, in reality, the true dose-response model is always unknown, and thus the linear model assumption could be violated and the dosing methods relying on such a specific assumption is risky. When the true underlying model is nonlinear, the subjects enrolled in the trial could be exposed to systematic dosing errors, and also future patients could be exposed to the risk of a poorly estimated dosing function. Therefore, a robust dose-finding design that can adapt for more general dose-response model settings than the linear model (2.1) is in need to develop. In this chapter, we propose a novel semiparametric adaptive design, which can not only determine doses adjusted for subjects' predicted clearance, but could also adapt for an unknown dose-response link function under a semiparametric model.

To generalize the linear model (2.1), we suppose that the relationship between the loga- 
rithm of AUC, dose and predicted clearance follows a nonlinear model as follows:

$$
y=g(\alpha+\beta x+\gamma z)+\epsilon, \quad \beta>0, \gamma \leq 0
$$

where $y, x$ and $z$ still respectively denote the AUC, continuous dose and predicted clearance on log-scale; $g(s)$ is an unknown increasing link function; and, $\epsilon$ is a normally distributed random error with zero mean and finite variance $\sigma^{2}$.

Actually, without loss of generality, we can further assume that $\alpha=0$ and $\beta=1$, because, by defining a new link $g^{*}(s)=g(\alpha+\beta s)$, the mean structure of Model (4.1) can be transformed to $E(y \mid x, z)=g^{*}\left(x+\frac{\gamma}{\beta} z\right)$ without changing any condition on the link function. Therefore, for identifiability, as long as the link is unknown, we assume the following nonlinear model in the rest of this dissertation:

$$
y=g(x+\phi z)+\epsilon, \quad \phi \leq 0 .
$$

Suppose that the true direction parameter is $\phi_{0}$, and the parameter ranges is a bounded closed interval $I_{\phi} \subset(-\infty, 0]$. Also, in the rest of this chapter, we denote the "index" as

$$
s=x+\phi z
$$

According to the conditions on the link, $s$ should take values within $I_{S}$, where $I_{S}$ is the compact domain of the link function $g($.$) .$

By assuming the single-index model (4.2), the number of unknown parameters is reduced by "absorbing" the intercept $\alpha$ and dose slope $\beta$ into the link function. And, more importantly, since $x$ is a stochastic regressor while $z$ is a non-random covariate, the model (4.2) bypasses the estimation for the dose slope $\beta$, and so avoids the need for special control on the dose convergence rate as the eigenvalue constraint (2.6) does. However, this does not 
mean that the estimation for model (4.2) would be easier to conduct, as it actually transfers the difficulty in estimation of $\beta$ to that of the link function $g($.$) . This point will be further$ discussed in Section 4.5.

\subsection{Isotonic Semiparametric Least Squares Estimation}

The model (4.1) and (4.2) are single-index models, which naturally extend the linear models, meanwhile overcomes the "curse of dimensionality" and lack of interpretability by linearly projecting all covariates into one single predictor. Over the past decades, the estimation methods for single-index models have been well studied in the community of semiparametric regression models. A brief review of these methods can be found in Chapter 6 of Hardle et al. [2004]. This section will introduce a two-stage iterative semiparametric estimation method for the single-index models, which will be recursively applied to estimate the dosing function in our proposed semiparametric adaptive design.

One commonly used approach to estimate the single-index models is the semiparametric least squares (SLS) method [Ichimura, 1993]. Generally speaking, SLS estimates $\phi$ by

$$
\hat{\phi}=\underset{\phi \in I_{\phi}}{\arg \min } \sum_{k=1}^{n}\left(y_{k}-g\left(x_{k}+\phi z_{k}\right)\right)^{2}
$$

where the unknown link function $g($.$) is viewed as a nuisance parameter and estimated by a$ consistent nonparametric smoother point-wisely.

Weisberg and Welsh [1994] independently propose a pseudo maximum likelihood version of SLS method under the generalized linear model setting with a missing link. Their iterative approach at first estimates the direction parameter $\phi_{0}$ using the canonical link, then estimates the link by a kernel smoother given the estimated direction parameter obtained from the first step, and then re-estimates the direction parameter via the pseudo maximum likelihood estimation with plugged-in kernel estimators. They solve a pseudo score equation by using 
the Newton-Raphson algorithm, and iterate between the second and third steps until certain convergence criterion is met, so as to obtain the final estimate of $\phi_{0}$.

Under the assumption that the response distribution is from a two-parameter exponential family, the score equation of maximum likelihood is the same as the normal equation of least squares. Thus, under a generalized linear model setting, the iterative approach by Weisberg and Welsh [1994] is essentially the same as SLS in terms of the estimation of direction parameter $\phi_{0}$. For our proposed semiparametric sequential design, the iterative estimation approach by Weisberg and Welsh [1994] has to be applied with several modifications to estimate the single-index model (4.2).

When applying SLS to estimate the model (4.2), besides the unknown link $g($.$) , the$ first order derivative $g^{\prime}($.$) is also involved in the pseudo score equation and thus needs$ to be estimated as well. Weisberg and Welsh [1994] use two separate kernel smoothers to estimate $g($.$) and g^{\prime}($.$) respectively, where the kernel estimate of g^{\prime}($.$) also contains the$ plugged-in kernel estimate of $g($.$) . In the early 1990s when Weisberg and Welsh [1994]$ proposed this method, it is also the period when the nonparametric regression methods are developed rapidly, for instance the local polynomial regression models. As introduced in Chapter 2 and 3 of Fan and Gijbels [1996], the local polynomial estimator not only keeps all advantages of the classic kernel smoothers, but also owns extra benefits over them, for example, simultaneously estimating the regression function and its derivatives, adapting to both fixed and random designs, and more accurate estimation on the boundary. Considering of these desirable properties, we thus modify the SLS [Weisberg and Welsh, 1994] by replacing the kernel estimators with the local linear estimator wherever the estimation for the link $g($. and its first order derivative $g^{\prime}($.$) is in need.$

Moreover, another major modification that we make to the nonparametric regression part of SLS [Weisberg and Welsh, 1994] is to incorporate the monotonicity of the unknown link function into the estimation procedure. The nonparametric regression methods, such as the 
kernel smoothing or local polynomial fit, only make use of the smoothness of the link function, but do not utilize the monotonicity at all, and thus cannot guarantee a monotone estimate. However, for reasonable interpretation of the dose-response model (4.2), the estimated link has to be increasing in the index $s=x+\phi z$. Therefore, we apply an isotonisation step [Mammen, 1991, Mukerjee, 1988] to the responses corresponding to the ordered indexes prior to the local linear regression, so as to ensure the resultant link function estimate is monotone. In brief, the first step is using the isotonic regression [Mukerjee, 1988] to obtain the isotonized version of $y_{i}$ onto the ordered indexes; then, the second step is applying the local linear fit to the pairs of isotonized responses and ordered indexes.

Algorithm 4 (Isotonic Semiparametic Least Squares Estimation) The isotonic semiparametic least squares (ISLS) estimation that we propose to sequentially estimate the model (4.2) is summarized as follows. Given $\left(y_{i}, x_{i}, z_{i}\right), i=1, \cdots, n$,

1. Assuming the canonical (identity) link at first, the model (4.1) is reduced to the linear model (2.1), so it is natural to apply least squares estimation to obtain $\left(\hat{\alpha}_{n}, \hat{\beta}_{n}, \hat{\gamma}_{n}\right)^{\prime}$. Now, the model (4.2) is actually $E(y \mid x, z)=g(x+\phi z)$ with a linear link $g(s)=\alpha+\beta s$ and $\phi=\gamma / \beta$. Then, an initial value of $\phi_{0}$ can be estimated by $\hat{\phi}_{0}=\hat{\gamma}_{n} / \hat{\beta}_{n}$, and the initial index values can be computed as $\hat{s}_{k}^{(0)}=x_{k}+\hat{\phi}_{0} z_{k}$, for $k=1, \cdots, n$.

2. For the $j$ th iteration $(j \geq 0)$, we first sort the index values to obtain the ordered indexes $\hat{s}_{(k)}^{(j)}=x_{k}+\hat{\phi}_{j} z_{k}, k=1, \cdots, n$, along with $y_{(k)}$ being the response corresponding to $\hat{s}_{(k)}^{(j)}$. Then, the isotonized responses can be obtained according to

$$
y_{k}^{*}=\max _{1 \leq L_{1} \leq k} \min _{k \leq L_{2} \leq n} \sum_{l=L_{1}}^{L_{2}} \frac{y_{(l)}}{L_{2}-L_{1}+1} \text {, for } k=1, \cdots, n \text {. }
$$

3. Apply the local linear fit to $\left(\hat{s}_{(1)}^{(j)}, y_{1}^{*}\right), \cdots,\left(\hat{s}_{(n)}^{(j)}, y_{n}^{*}\right)$, and obtain the point-wise estimates of link function and its first order derivative at $\hat{s}_{(k)}^{(j)}$ for $k=1, \cdots, n$. Particularly, for 
any fixed $s \in I_{S}$, the local linear estimator of $\left(g(s), g^{\prime}(s)\right)$ is

$$
\left(\hat{g}_{n}(s), \hat{g}_{n}^{\prime}(s)\right)^{\prime}=\left(W_{n}^{\prime}(s) \Omega_{n}(s) W_{n}(s)\right)^{-1} W_{n}^{\prime}(s) \Omega_{n}(s) Y_{n}^{*}
$$

where $W_{n}(s)=\left(\begin{array}{cc}1 & \hat{s}_{(1)}^{(j)}-s \\ \vdots & \vdots \\ 1 & \hat{s}_{(n)}^{(j)}-s\end{array}\right)$ and $\Omega_{n}(s)=\left(\begin{array}{ccc}K_{h}\left(\hat{s}_{(1)}^{(j)}-s\right) & \cdots & 0 \\ \vdots & \ddots & \vdots \\ 0 & \cdots & K_{h}\left(\hat{s}_{(n)}^{(j)}-s\right)\end{array}\right)$ are respectively the design matrix and diagonal kernel weight matrix of local linear regression at the point $s, Y_{n}^{*}=\left(y_{1}^{*}, \cdots, y_{n}^{*}\right)^{\prime}$ is the isotonized response vector, the kernel function $K($.$) is a symmetric light-tail probability density function and K_{h}()=$. $\frac{1}{h} K(. / h)$, and $h$ is a bandwidth controlling the size of the local neighborhood and selected at the optimal rate such that $h=O\left(n^{-1 / 5}\right)$. For simplicity, we denote $V_{n}(s)=$ $W_{n}^{\prime}(s) \Omega_{n}(s) W_{n}(s)$ and $U_{n}(s)=W_{n}^{\prime}(s) \Omega_{n}(s) Y_{n}^{*}$ in the rest of this chapter.

4. Then, the estimate of $\phi_{0}$ will be updated by the solution to the pseudo score equation

$$
\hat{\mathcal{S}}_{n}(\phi)=\frac{1}{n} \sum_{k=1}^{n} z_{(k)} \hat{g}_{n}^{\prime}\left(s_{(k)}(\phi)\right)\left\{y_{k}^{*}-\hat{g}_{n}\left(s_{(k)}(\phi)\right)\right\}=0
$$

where $s_{k}(\phi)=x_{k}+\phi z_{k}$ for $k=1, \cdots, n$, and the local linear estimators $\hat{g}_{n}($.$) and$ $\hat{g}_{n}^{\prime}($.$) are plugged in to substitute the unknown link and its first order derivative. The$ Newton-Raphson algorithm is applied to solve the equation (4.5) as follows:

$$
\hat{\phi}_{j+1}=\hat{\phi}_{j}+\hat{A}_{j} \frac{1}{n} \sum_{k=1}^{n} z_{(k)} \hat{g}_{n}^{\prime}\left(\hat{s}_{(k)}^{(j)}\right)\left\{y_{k}^{*}-\hat{g}_{n}\left(\hat{s}_{(k)}^{(j)}\right)\right\} J_{a}\left(\left|V_{n}\left(\hat{s}_{(k)}^{(j)}\right)\right|\right)
$$

where $\hat{A}_{j}^{-1}=\frac{1}{n} \sum_{k=1}^{n} z_{(k)}^{2}\left(\hat{g}_{n}^{\prime}\left(\hat{s}_{(k)}^{(j)}\right)\right)^{2} J_{a}\left(\left|V_{n}\left(\hat{s}_{(k)}^{(j)}\right)\right|\right), z_{(k)}$ is ordered up to $\hat{s}_{(k)}^{(j)}$ for $k=$ $1, \cdots, n$, and $\left|V_{n}\left(\hat{s}_{(k)}^{(j)}\right)\right|$ is the determinant of the matrix. The function $J_{a}(t)$ is a smooth version of the indicator function that targets at stabilizing the local linear estimator at points where $\left|V_{n}\left(\hat{s}_{(k)}^{(j)}\right)\right|$ is close to zero. According to Weisberg and 
Welsh [1994], we choose

$$
\begin{aligned}
& J_{a}(t)=J(2(t-a) / b-1) 1_{\{a \leq t<a+b\}}+1_{\{t \leq a+b\}}, \text { for some small } b>0, \text { and } \\
& J(x)=\frac{15}{16}\left(\frac{1}{5} x^{5}-\frac{2}{3} x^{3}+x+\frac{8}{15}\right), \text { for }-1 \leq x \leq 1,
\end{aligned}
$$

where the tuning constant $a$ is required to decrease to 0 more slowly than the bandwidth $h$, which is expressed by $a=O\left((\log \log n)^{-1}\right)$. Such smoothing modification is common and necessary for nonparametric regression estimation, as a small determinant on the denominator of (4.4) would largely bias the estimator. The smoothed indicator function actually allows only those points at which the local linear estimation is stable to fully contribute to the correction term updated in the iterative algorithm.

5. Repeat Step 2 through 4 until some convergence criterion on $\hat{\phi}$ is satisfied. In this research, the Cauchy convergence criterion is applied. Specifically, if there exists a fixed number $N^{*}$ such that

$$
\left|\hat{\phi}_{j}-\hat{\phi}_{j+1}\right|<\xi, \quad \text { for } j=N^{*}, \cdots, N^{*}+m
$$

where the threshold $\xi$ and step-length $m$ are pre-selected, then the iterative estimation procedure is considered convergent.

Based on the iterative estimation approach ISLS, we develop a novel individualized dosefinding design, the semiparametric link-adapted recursion (SLAR), which can adapt for the unknown link function under the semiparametric model (4.2). 


\subsection{Semiparametric Link-Adapted Recursion Design}

The primary goal is still looking for a sequential design such that $E(y \mid x, z)$ can reach the target level $t_{0}$. Under the single-index model (4.2), the trial objective then becomes the estimation of the following target dosing function:

$$
\theta(z)=s_{0}-\phi_{0} z, \text { for any given } z \in I_{z},
$$

where $s_{0}=g^{-1}\left(t_{0}\right)$. Intuitively, the aforementioned conditions on the link function $g($.$) is to$ guarantee the invertibility of $g($.$) at t_{0}$, in other words, the existence and uniqueness of $s_{0}$.

In brief, the essential idea of the semiparametric link-adapted recursion (SLAR) design is, iteratively estimating $s_{0}$ and $\phi$ via using ISLS to the current $i$ observations, then assigning an estimated dose $\hat{\theta}_{i}\left(z_{i+1}\right)=\hat{s}_{0 i}-\hat{\phi}_{i} z_{i+1}$ to the $(i+1)$ th subject, and repeating this procedure for next subjects until a pre-specified sample size is reached. This sequential procedure involves recursive estimation of the unknown link function $g($.$) and the direction parameter \phi_{0}$ as the trial proceeds. In order to ensure a consistent dose sequence, it is of core interests to obtain precise estimates of not only the direction parameter $\phi_{0}$ but also the inverse link at $t_{0}$, that is, $s_{0}=g^{-1}\left(t_{0}\right)$.

The semiparametric link-adapted recursion (SLAR) is a two-stage design: the first stage with a sample size $n_{1}\left(n_{0}<n_{1} \leq 40\right)$ applies the repeated least squares procedure, while the second stage is actually the proposed SLAR design. One reason for the necessity of the first stage is that, the nonparametric regression fitting involved in ISLS typically requires certain amounts of data points to guarantee the precision and reliability of nonparametric estimation. Also, another reason is that, the first stage can lead to an data-driven initial estimate for the direction parameter $\phi_{0}$, which is necessary to launch the iterative estimation of the link and $\phi_{0}$. Specifically, the first step of ISLS postulates the canonical link, which is the identity link under the normal distribution assumption. The model (4.1) with the identity link is 
reduced to the linear model (2.1), and thus, the repeated least squares procedure becomes a natural choice for the design. In fact, the choice of the switching point $\left(n_{1}\right)$ is crucial for SLAR, because, intuitively, an early switch would cause comparatively less accurate nonparametric estimates due to the small sample size, and further affect the estimation precision of ISLS. The calibration of selecting $n_{1}$ for SLAR will be discussed in subsequent numerical simulations.

At the end of the first stage, the least squares fit to $\left(y_{i}, x_{i}, z_{i}\right), i=1, \cdots, n_{1}$, leads to the estimates $\left(\hat{\alpha}_{n_{1}}, \hat{\beta}_{n_{1}}, \hat{\gamma}_{n_{1}}\right)^{\prime}$, which is used to trigger the second stage. Specifically, the initial estimate of $\phi_{0}$ suggested by the first stage is

$$
\tilde{\phi}_{n_{1}}=\hat{\gamma}_{n_{1}} / \hat{\beta}_{n_{1}}
$$

which is truncated from up by 0 , so as to ensure that AUC decreases in the predicted clearance given the increasing link function. Then, the initial "index" values can be calculated as $\tilde{s}_{k}^{\left(n_{1}\right)}=x_{k}+\tilde{\phi}_{n_{1}} z_{k}$ for $k=1, \cdots, n_{1}$, which are used to initiate the local linear regression for the link function at the beginning of the second stage.

\section{Algorithm 5 (SLAR)}

For the $(i+1)$ th subject, $i=n_{1}, \cdots, n$, given $\left\{\left(y_{k}, x_{k}, z_{k}\right)\right\}_{k=1}^{i}$ and $\hat{\phi}_{i-1}$, where, especially, the initial estimate of $\phi$ at the $n_{1}$ step is $\tilde{\phi}_{n_{1}}(4.9)$,

1. First of all, the initial indexes at the $(i+1)$ th step are computed as $\tilde{s}_{k}^{(i)}=x_{k}+\tilde{\phi}_{i} z_{k}$, $k=1, \cdots, i$, where $\tilde{\phi}_{i}=\hat{\phi}_{i-1}$. Then, we conduct the isotonisation step (4.3) to the responses onto the ordered indexes, so as to obtain the monotone observations $\left(\tilde{s}_{(1)}^{(i)}, y_{1}^{*}\right), \cdots,\left(\tilde{s}_{(i)}^{(i)}, y_{i}^{*}\right)$.

2. At the second, we perform the following iterative ISLS procedure.

i) Apply the local linear fit to $\left(\tilde{s}_{(1)}^{(i)}, y_{1}^{*}\right), \cdots,\left(\tilde{s}_{(i)}^{(i)}, y_{i}^{*}\right)$, and obtain $\left(\hat{g}_{i}\left(\tilde{s}_{(k)}^{(i)}\right), \hat{g}_{i}^{\prime}\left(\tilde{s}_{(k)}^{(i)}\right)\right)$ and the matrix $V_{i}\left(\tilde{s}_{(k)}^{(i)}\right)$ by $(4.4)$ for $k=1, \cdots, i$. 
ii) Update the estimate of $\phi_{0}$ by the root to the pseudo score equation (4.5) that is solved by the Newton-Raphson algorithm (4.6):

$$
\hat{\phi}_{i}=\tilde{\phi}_{i}+\frac{\hat{A}_{i}}{i} \sum_{k=1}^{i} z_{(k)} \hat{g}_{i}^{\prime}\left(\tilde{s}_{(k)}^{(i)}\right)\left\{y_{k}^{*}-\hat{g}_{i}\left(\tilde{s}_{(k)}^{(i)}\right)\right\} J_{a}\left(\left|V_{i}\left(\tilde{s}_{(k)}^{(i)}\right)\right|\right)
$$

where $\hat{A}_{i}^{-1}=\frac{1}{i} \sum_{k=1}^{i} z_{(k)}^{2}\left(\hat{g}_{i}^{\prime}\left(\tilde{s}_{(k)}^{(i)}\right)\right)^{2} J_{a}\left(\left|V_{i}\left(\tilde{s}_{(k)}^{(i)}\right)\right|\right)$.

iii) Update the indexes with $\hat{\phi}_{i}(4.10)$, such that

$$
\hat{s}_{k}^{(i)}=x_{k}+\hat{\phi}_{i} z_{k} \text { for } k=1, \cdots, i
$$

and then iterate between Step (2.i) and (2.ii) until the Cauchy criterion (4.7) on $\hat{\phi}_{i}$ is satisfied.

Upon the above iteration converges, the estimates of $\phi_{0}$, indexes and link function at stage $i$ are respectively denoted as $\hat{\phi}_{i}, \hat{s}_{k}^{(i)}$, and $\left(\hat{g}_{i}\left(\hat{s}_{(k)}^{(i)}\right), \hat{g}_{i}^{\prime}\left(\hat{s}_{(k)}^{(i)}\right)\right)$ for $k=1, \cdots, i$.

3. At the third, given $\left\{\hat{s}_{(k)}^{(i)}, \hat{g}_{i}\left(\hat{s}_{(k)}^{(i)}\right)\right\}_{k=1}^{i}$, we search for the root to $t_{0}=\hat{g}_{i}(s)$ by using a numerical root-searching algorithm. The choice on the algorithm is determined dynamically, depending on whether the target level $t_{0}$ sits in the interval $\left[\hat{g}_{i}\left(\hat{s}_{(1)}^{(i)}\right), \hat{g}_{i}\left(\hat{s}_{(i)}^{(i)}\right)\right]$.

i) If $\hat{g}_{i}\left(\hat{s}_{(1)}^{(i)}\right) \leq t_{0} \leq \hat{g}_{i}\left(\hat{s}_{(i)}^{(i)}\right)$, a Bisection algorithm will be applied;

ii) If $t_{0}<\hat{g}_{i}\left(\hat{s}_{(1)}^{(i)}\right)$, an Uphill searching algorithm will be applied;

iii) If $t_{0}>\hat{g}_{i}\left(\hat{s}_{(i)}^{(i)}\right)$, a Downhill searching algorithm will be applied.

As a result, the root $\hat{s}_{0 i}$ to $t_{0}=\hat{g}_{i}(s)$ is the estimate of $s_{0}=g^{-1}\left(t_{0}\right)$ at step $i$.

4. Given $\hat{s}_{0 i}$ and $\hat{\phi}_{i}$, the dosing function is estimated by

$$
\hat{\theta}_{i}(z)=\hat{s}_{0 i}-\hat{\phi}_{i} z, \quad \text { for any fixed } z \in I_{z} \text {. }
$$


The dose assigned to the $(i+1)$ th subject will be

$$
x_{i+1}=\min \left\{\max \left\{\hat{\theta}_{i}\left(z_{i+1}\right), x_{\min }\right\}, x_{\max }\right\} .
$$

5. Once $y_{i+1}$ is observed, the trial proceeds to the next subject and repeats Step 1 through 4. The sequential procedure continues until the sample size reaches $n$. At the trial's end, the estimated dosing function $\hat{\theta}_{n+1}(z)$ will be recommended to future patients.

The crucial steps of implementing SLAR is the Newton-Raphson and root-searching algorithms, as they respectively estimate the slope and intercept of the true dosing function (4.8). Thus, it would be necessary to introduce the Bisection, Downhill and Uphill searching algorithms specifically.

\section{Algorithm 6 (Bisection)}

Given $\left\{\hat{s}_{(k)}^{(i)}, \hat{g}_{i}\left(\hat{s}_{(k)}^{(i)}\right)\right\}_{k=1}^{i}$, if $\hat{g}_{i}\left(\hat{s}_{(1)}^{(i)}\right) \leq t_{0} \leq \hat{g}_{i}\left(\hat{s}_{(i)}^{(i)}\right)$, then we respectively set the starting lower and upper limits of the search as $\underline{s}=\hat{s}_{(1)}^{(i)}$ and $\bar{s}=\hat{s}_{(i)}^{(i)}$.

1. For the $j$ th iteration, $j \geq 1$, we at first let $s_{t j}=(\underline{s}+\bar{s}) / 2$, and perform local linear fit at $s_{t j}$ using $\left(\hat{s}_{(k)}^{(i)}, y_{k}^{*}\right), k=1, \cdots, i$, so as to obtain $\hat{g}_{i}\left(s_{t j}\right)$.

2. If $\hat{g}_{i}\left(s_{t j}\right)>t_{0}$, then we update $\bar{s}$ with $s_{t j}$ and repeat Step 1 ; if $\hat{g}_{i}\left(s_{t j}\right)<t_{0}$, then we update $\underline{s}$ with $s_{t j}$ and repeat Step 1 .

3. Iterate Step 1 through 2 until $\hat{g}_{i}\left(s_{t j}\right)=t_{0}$ for some $s_{t j}$. At last, the finally updated $s_{t j}$ is the root to $t_{0}=\hat{g}_{i}(s)$, that is $\hat{s}_{0 i}$.

The prerequisites of implementing Bisection algorithm include that, $\hat{g}_{i}(s)$ is increasing in $s \in\left[\hat{s}_{(1)}^{(i)}, \hat{s}_{(i)}^{(i)}\right]$, and $t_{0}$ sits in the interval of $\left[\hat{g}_{i}\left(\hat{s}_{(1)}^{(i)}\right), \hat{g}_{i}\left(\hat{s}_{(i)}^{(i)}\right)\right]$. The former can be guaranteed by the isotonisation step prior to the local linear regression, but the latter cannot be ensured by the estimation procedure. Actually, if the initial estimate $\hat{\phi}_{n_{1}}$ is substantially biased away 
from the true value $\phi_{0}$, it could cause that $t_{0}$ falls out of the interval of $\left[\hat{g}_{i}\left(\hat{s}_{(1)}^{(i)}\right), \hat{g}_{i}\left(\hat{s}_{(i)}^{(i)}\right)\right]$. At this case, if $t_{0}<\hat{g}_{i}\left(\hat{s}_{(1)}^{(i)}\right)$, a Downhill searching algorithm should be used to replace the Bisection algorithm; or, if $t_{0}>\hat{g}_{i}\left(\hat{s}_{(i)}^{(i)}\right)$, an Uphill searching algorithm should be used instead. In brief, the Downhill algorithm is, to search with a step-size for such a point $s_{t}$ that $\hat{g}_{i}\left(s_{t}\right)$ the first time downcrosses the target $t_{0}$, and then switch to the Bisection algorithm by setting $s_{t}$ as the initial lower limit $\underline{s}$; and, likewise for the Uphill searching algorithm.

Motivated by the need for an individualized dosing algorithm that adapts for an unknown link, SLAR is proposed to compete with the repeated least squares procedure. Under a semiparametric model, SLAR targets at suggesting more accurate doses within a trial and leading to more precisely estimated dosing function at a trial's end than RLSEVC does. In fact, as the second stage, SLAR relies on the performance of RLSEVC in the first stage.

The repeated least squares procedure, especially RLSEVC, plays an important role in providing a reliable initial estimate $\tilde{\phi}_{n_{1}}(4.9)$ to launch the second stage of SLAR. As a crucial part of SLAR algorithm, the Newton-Raphson algorithm results in the estimate of $\phi_{0}$, but its performance somehow depends on the initial value $\tilde{\phi}_{n_{1}}$. A very biased initial value could cause that, the resultant estimates $\hat{s}_{0 i}$ and $\hat{\phi}_{i}$ are also biased away from the true values, or even the Newton-Raphson algorithm does not converge at all. If $\tilde{\phi}_{n_{1}}$ was too far away from the true value $\phi_{0}$, then the local linear fit would take place at a place far away from the true location, and thus lead the solution of $t_{0}=\hat{g}_{i}(s)$ to deviate from the true value $s_{0}=g^{-1}\left(t_{0}\right)$. The Bisection algorithm is fast-converging and stable though, it cannot correct any information regarding the location of fitting the nonparametric curve. As a result, the dosing function would be poorly estimated. The use of repeated least squares procedure, especially RLSEVC, in the first stage can largely avoid the occurrence of a very biased $\tilde{\phi}_{n_{1}}$. On one hand, due to wrong model assumption, the resultant estimates of $\phi_{0}$ and $s_{0}$ by RLSEVC may not be accurate enough, and so need further improvement by SLAR; however, on the other hand, they are reliable enough to an ideal initial value for the second 
stage of SLAR.

Moreover, besides the Newton-Raphson algorithm, another important influential part for the performance of SLAR is the local linear fit. Due to the iterative estimation manner of $\phi_{0}$ and $g($.$) , the local linear fit not only directly determines the search for s_{0}$, but also has influence on estimating $\phi_{0}$ through the correction term in (4.10). An unstable local linear estimates of $g($.$) and g^{\prime}($.$) would attribute to an unstable correction term (especially for$ $\left.\hat{A}_{i}\right)$ in updating $\hat{\phi}_{i}$, which could cause the iterative algorithm not to converge. To stabilize the local linear estimates, we calibrate the choice of the switching point $n_{1}$, which will be discussed in subsequent section of simulations, and particularly use a dynamic way to select the bandwidth.

Specifically, the rate of bandwidth is chosen at the optimal rate $\mathrm{O}\left(n^{-1 / 5}\right)$ (see Comment 1 (4.29) in Section 4.5), while a constant is empirically selected to multiply on the rate

$$
h_{n}=0.25\left(\hat{s}_{(n)}^{(n)}-\hat{s}_{(1)}^{(n)}\right) n^{-1 / 5}
$$

so as to ensure certain amount of observations in a local neighborhood. Moreover, if some "isolated indexes" are detected, the bandwidth would be enlarged enough so that at least two data points are contained in each isolated index's local neighborhood. The existence of these isolated indexes is due to the small sample size and the tendency that the indexes concentrate around $s_{0}$. The estimated indexes at stage $i$ are actually

$$
\hat{s}_{k}^{(i)}=x_{k}+\hat{\phi}_{i} z_{k}=\hat{s}_{0, k-1}+\left(\hat{\phi}_{i}-\hat{\phi}_{k-1}\right) z_{k}, \text { for } k=1, \cdots, i
$$

As long as $\hat{\phi}_{i}$ and $\hat{s}_{0 i}$ are consistent for $\phi_{0}$ and $s_{0}$ respectively, the index sequence $\left\{\hat{s}_{k}^{(i)}\right\}$ should approach $s_{0}$ regardless the covariate values $z_{k}$, especially for those in late trial. Since our major interest regarding the nonparametric regression is the estimation at the point $\left(s_{0}, t_{0}\right)$, rather than the entire curve, the aforementioned phenomenon can benefit the search 
for $s_{0}$ by making most data surround the fitting location of interest. However, it also has a side effect that several early indexes would be "isolated" from the majority, in the sense that their local neighborhoods defined by the bandwidth (4.14) have no data points other than themselves. The local linear fitting at such isolated indexes could result in an unstable

correction term in updating $\hat{\phi}_{i}$, because the $\hat{A}_{i}^{-1}$ could be very close to zero. Our proposed dynamic bandwidth selection succeeds in overcoming this issue. In fact, when RLSEVC is utilized as the first stage, the effect of eigenvalue constraint in spreading design points also can certainly avoid the indexes to concentrate around $s_{0}$ too rapidly.

\subsection{Applications of Semiparametric Link-Adapted Re- cursion}

\subsubsection{Fitting Semiparametric Models to Irinotecan Data}

In the numerical evaluation of SLAR, the criteria measuring the performance of designs are still the same as those defined in Section 3.1, that is, the dosing cost (3.1) and ISE (3.2). The first step of numerical simulations would be to select suitable true models that will be used to generate the responses. So, it is of interest to fit certain single-index models to the irinotecan data, so as to obtain the "suggestions" on the model from the data.

Looking into the specific single-index model (4.2), the parameter $\phi$ determines the "location" where the nonlinear regression between the response $y$ and index $s=x+\phi z$ happens. Without prior knowledge on clinical meaning of the index $s$, we should not suppose any specific location range of the index, but can dig that information out from the irinotecan data. The method that we use to find $\phi_{0}$ is similar to maximizing a nonparametric profile likelihood on $\phi$.

Given the irinotecan data $\left\{\left(y_{i}, x_{i}, z_{i}\right)\right\}_{i=1}^{n}$ and any fixed $\phi \leq 0$, the indexes $s_{i}=x_{i}+\phi z_{i}$ 
for $i=1, \cdots, n$, are computed at first; then, a nonparametric kernel smoothing between $\left\{y_{i}\right\}$ and $\left\{s_{i}\right\}$ is performed to obtain the kernel estimates $\left\{\tilde{g}\left(s_{i}\right)\right\}$ point-wisely; at last, the residual squares sum (RSS) of the nonparametric regression fits is computed as follows

$$
\operatorname{RSS}(\phi)=\sum_{i=1}^{n}\left\{y_{i}-\tilde{g}\left(s_{i}(\phi)\right)\right\}^{2}
$$

Figure 4.1: Profile of Residual Square Sums from Kernel Smoothing

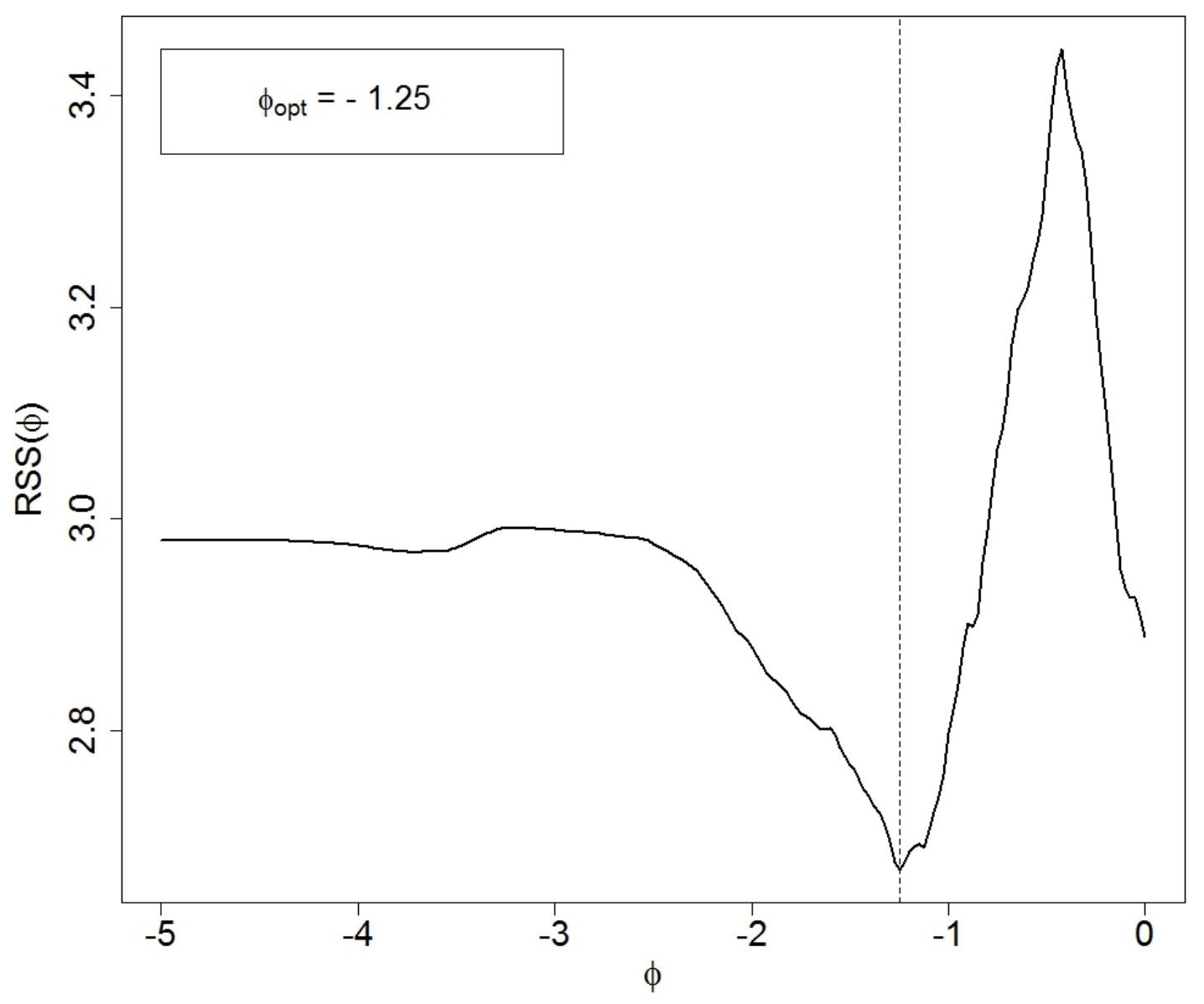

By letting $\phi$ vary through at sufficiently many values within $(-\infty, 0]$ and correspondingly performing the kernel regression at each fixed $\phi$, a profile of $\operatorname{RSS}(\phi)$ can be obtained then, as shown in Figure 4.1. The optimal $\phi$ suggested by the irinotecan data is the one that 
minimizes $\operatorname{RSS}(\phi)$ over $\phi \in(-\infty, 0]$. As shown by Figure 4.1, there is a unique minimizer of $\operatorname{RSS}(\phi)$, which is $\phi_{\text {opt }}=-1.25$. We will set the true parameter as $\phi_{0}=-1.25$ in the subsequent numerical simulations of this dissertation.

Given $\phi_{0}=-1.25$, we fit several specific nonlinear link functions to the data $\left\{\left(y_{i}, s_{i}\right)\right\}_{i=1}^{n}$, where now $s_{i}=x_{i}+\phi_{0} z_{i}$. The first one is a commonly used model in the pharmacokinetics and pharmacodynamics (PKPD) theory, the $\mathrm{E}_{\max }$ model [Kirby et al., 2011]. The $\mathrm{E}_{\max }$ models usually postulate the following nonlinear association between a response $y$ and a predictor D, which could be the dosage or any clinically important biomarker.

$$
y=\mathrm{E}_{0}+\frac{\mathrm{E}_{\max } \mathrm{D}^{\zeta}}{\mathrm{ED}_{50}^{\zeta}+\mathrm{D}^{\zeta}}+\epsilon,
$$

where $\mathrm{E}_{0}, \mathrm{E}_{\max }, \mathrm{ED}_{50}$ and $\zeta$ are unknown parameters and random noise $\epsilon$ is assumed to normally distributed with zero mean and finite variance $\sigma^{2}$. Particularly, $\mathrm{E}_{0}$ represents the minimum possible response corresponding to $\mathrm{D}=0, \mathrm{E}_{0}+\mathrm{E}_{\max }$ represents the maximum possible response as $\mathrm{D}$ increases to $+\infty, \mathrm{ED}_{50}$ is the predictor level that produces half of the $\mathrm{E}_{\max }$ effect and actually controls the location of the curve, and $\zeta$ is the slope and controls the steepness of the curve. All the parameters are positive, so that the mean response would be increasing in the predictor. A typical $\mathrm{E}_{\max }$ curve shape can found in Figure 4.2 (b).

If we suppose the true link function $g($.$) is an \mathrm{E}_{\text {max }}$ link, such that

$$
g(s)=\mathrm{E}_{0}+\frac{\mathrm{E}_{\max } \mathrm{s}^{\zeta}}{\left(\mathrm{Es}_{50}\right)^{\zeta}+\mathrm{s}^{\zeta}}, \quad \text { for } s \in I_{S},
$$

then, by applying the nonlinear least squares fit to the data $\left\{\left(y_{i}, s_{i}\right)\right\}_{i=1}^{n}$, the estimated model (p-values) is obtained as $\hat{\mathrm{E}}_{0}=2.92(\mathrm{p}<0.0001), \hat{\mathrm{E}}_{\max }=0.36(\mathrm{p}=0.3), \hat{\mathrm{Es}}_{50}=2.28(\mathrm{p}$ $<0.0001), \hat{\zeta}=19.28(\mathrm{p}=0.57)$ and $\hat{\sigma}=0.2555$, with a significant model (Wald test $\mathrm{p}<$ 0.0001). The estimated $\mathrm{E}_{\max }$ link is shown in Figure 4.2 (b). Its model fitting (see Figure 4.2 (e)) seems not much better $\left(R^{2}=0.126\right)$ than a linear link (see Figure $4.2(\mathrm{a})$ and $(\mathrm{d})$ ), 

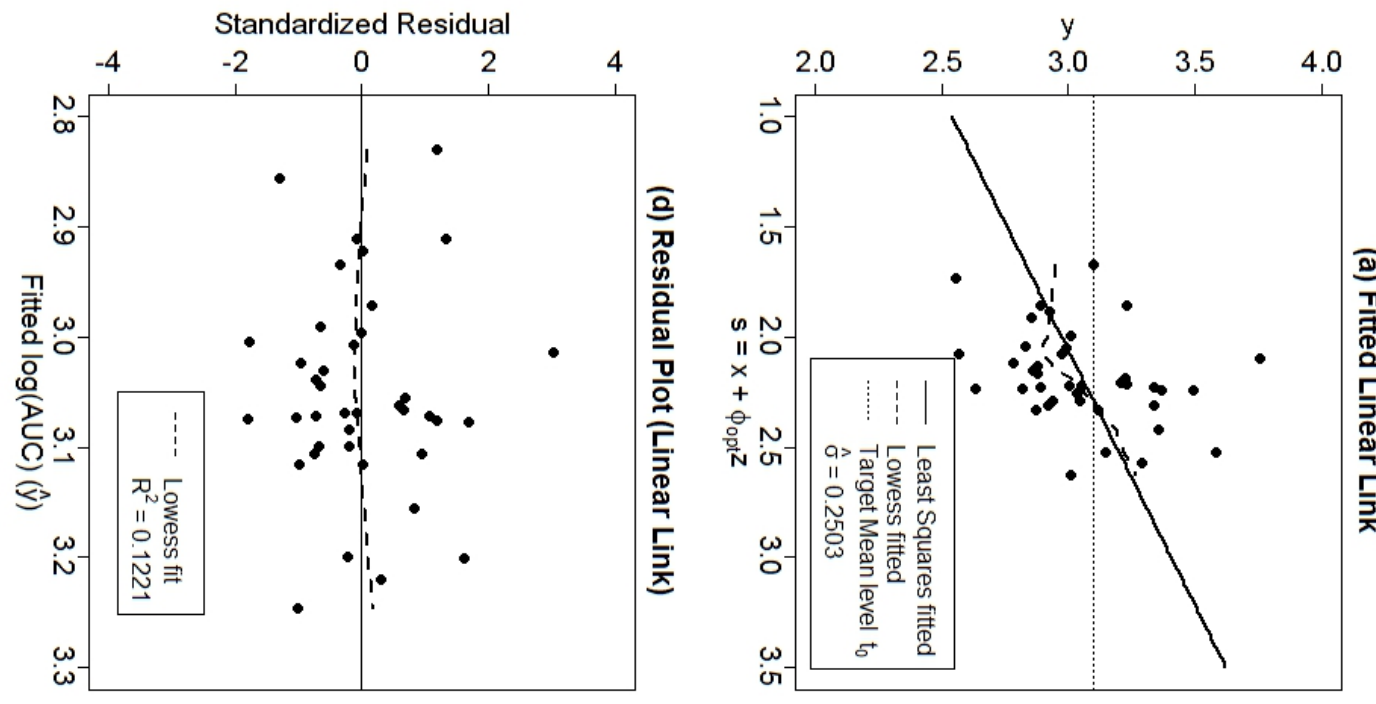

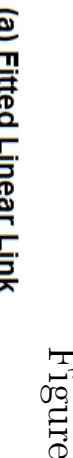
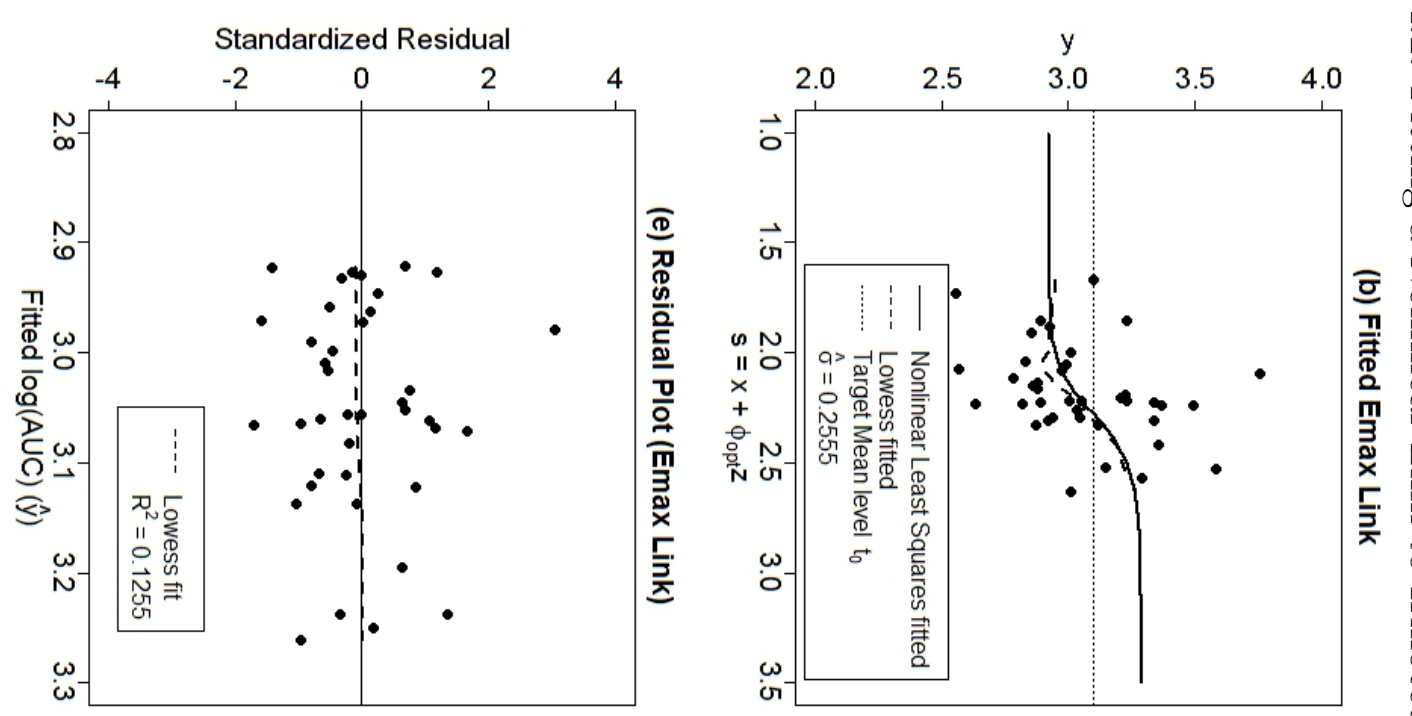

$\stackrel{+}{i}$
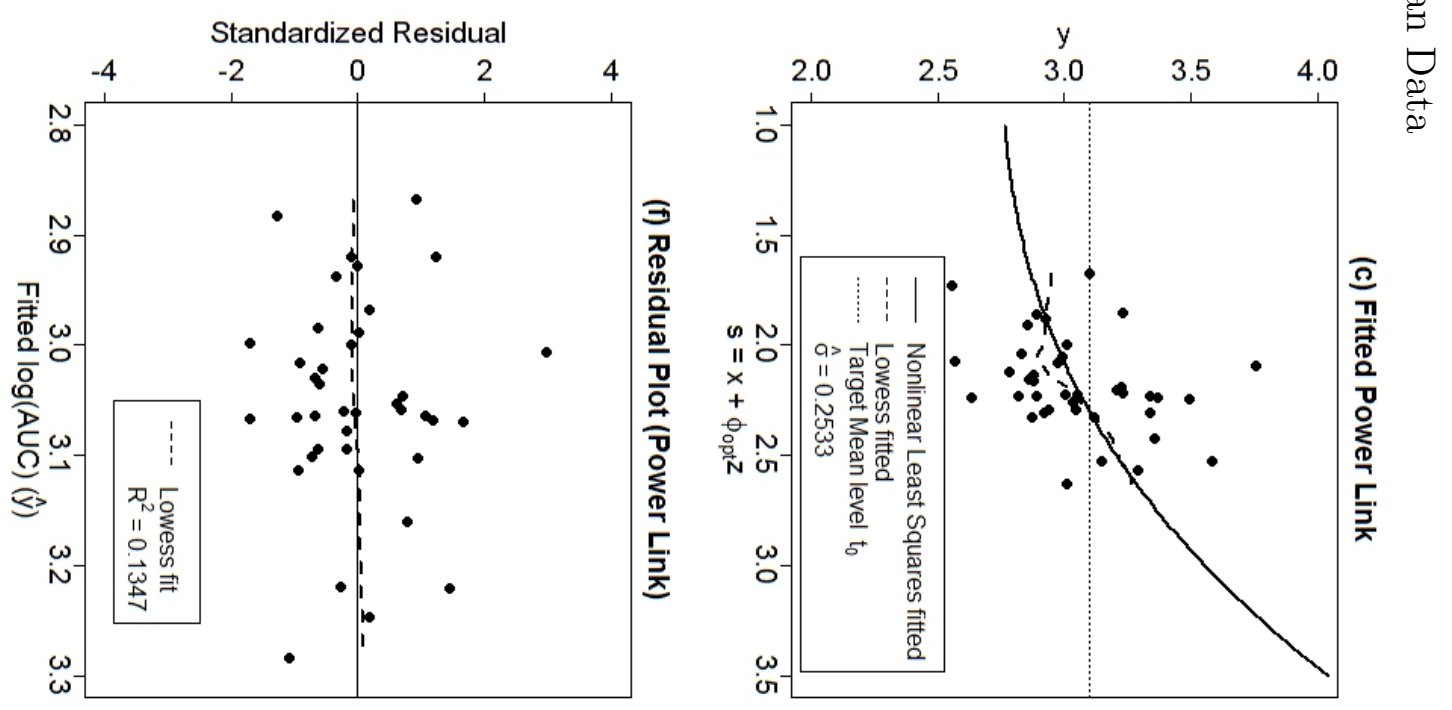
as the data themselves display no significant linear or nonlinear pattern because of its small range and limited sample size.

Besides the $\mathrm{E}_{\max }$ link, we also use the nonlinear least squares to fit a power link function, such that

$$
g(s)=\psi_{0}+\psi_{1}\left(s-\psi_{3}\right)^{\psi_{2}}, \quad \text { for } s \in I_{S}
$$

The estimates are $\hat{\psi}_{0}=2.74(\mathrm{p}<0.0001), \hat{\psi}_{1}=0.06(\mathrm{p}=0.85), \hat{\psi}_{2}=2.71(\mathrm{p}=0.62)$ and $\hat{\sigma}=0.2533$, with a significant model (Wald test $\mathrm{p}<0.0001$ ), when the location $\phi_{3}$ is fixed at 0.35 (otherwise the Newton-Raphson algorithm in the nonlinear least squares fit does not converge). The power link model appears to fit the data slightly better $\left(R^{2}=0.135\right)$ than the $\mathrm{E}_{\max }$ link does (see Figure 4.2 (f)), however, due to the non-patterned data, it still does not display persuasive evidence that the power link fits the data perfectly.

Figure 4.3: Nonlinear Links used in Simulations

(a) Emax Link

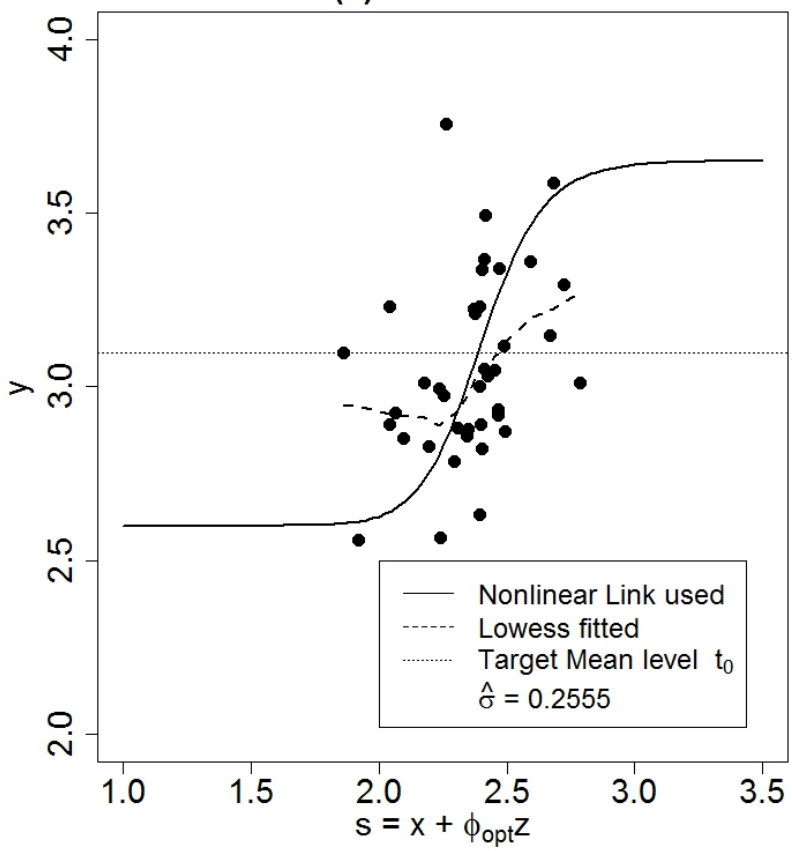

(b) Power Link

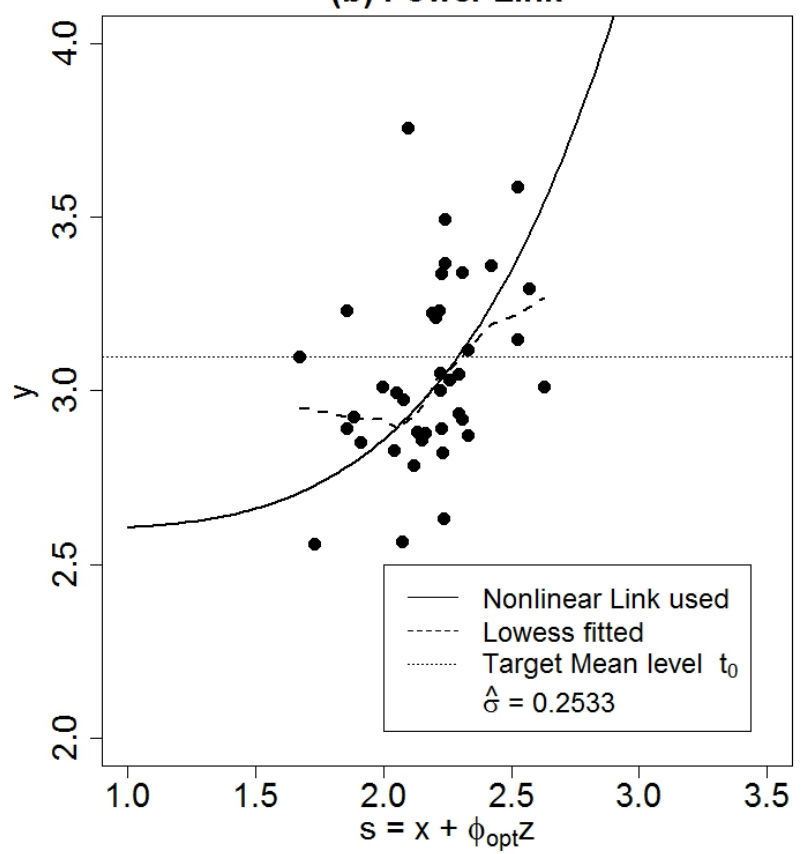

However, the choice of true models used to generating responses in simulations should not depend on specific data sets completely, otherwise the simulation results would also depend 
on the particular data and lose generality. Therefore, in the subsequent simulations for evaluating the performance of SLAR, we use the $\mathrm{E}_{\max }$ and power links, but with some minor modifications on the parameter estimates to make the nonlinear shape of the curves much clearer. Specifically, given $\phi_{0}=-1.25$, the parameters of $\mathrm{E}_{\max }$ link are selected respectively as $\mathrm{E}_{0}=2.6, \mathrm{E}_{\max }=1.05, \mathrm{Es}_{50}=2.4, \zeta=20$ and $\sigma=0.2555$; meanwhile, the parameters of power link are respectively $\psi_{0}=2.6, \psi_{1}=0.035, \psi_{2}=4, \phi_{3}=0.35$ and $\sigma=0.2533$ (see Figure 4.3). The parameters regarding the mean structures are modified slightly to highlight their nonlinear shape, provided that the data do not show any significant pattern. While the location parameter $\phi_{0}$ and the dispersion $\sigma$ are kept the same as those obtained from the irinotecan data, so as to maintain those information dug out from the data.

\subsubsection{An Illustrative Trial}

In this section, we illustrate the SLAR's advantage in improving the estimation precision over RLSEVC by simulating an illustrative trial. Similar to the sample trial in Section 3.4, we simulate a trial of $n=40$ subjects by utilizing RLSEVC connected with SLAR at its end $\left(n_{1}=40\right)$, where $\left(\delta_{1}, \delta_{2}\right)=(0.3,3.5)$ for RLSEVC and the coherence restriction is applied as a default. The true model to generate responses is the Emax link model (4.17) mentioned at the end of Section 4.4.1.

The left plot in Figure 4.4 presents the true mean response function against the index, along with the two estimated mean curves by RLSEVC and SLAR respectively. The focus of curve estimation is indeed at the point $\left(s_{0}, t_{0}\right)$, where the curve crosses the target mean level of the response. The curve estimated by RLSEVC crosses $t_{0}$ at $\hat{s}_{0 n}=2.71$. At the end of the RLSEVC trial, the additional step of SLAR improves the estimate to $\hat{s}_{0 n}=2.41$, which is closer to the true value $s_{0}=2.39$ for this example. This improvement in estimating $s_{0}$ is mainly sourced from the improvement in estimating $\phi_{0}$ by SLAR over RLSEVC. The estimate of $\phi_{0}$ by SLAR is $\hat{\phi}_{n}=-1.24$, which is almost identical to the true value $\phi_{0}=-1.25$. 


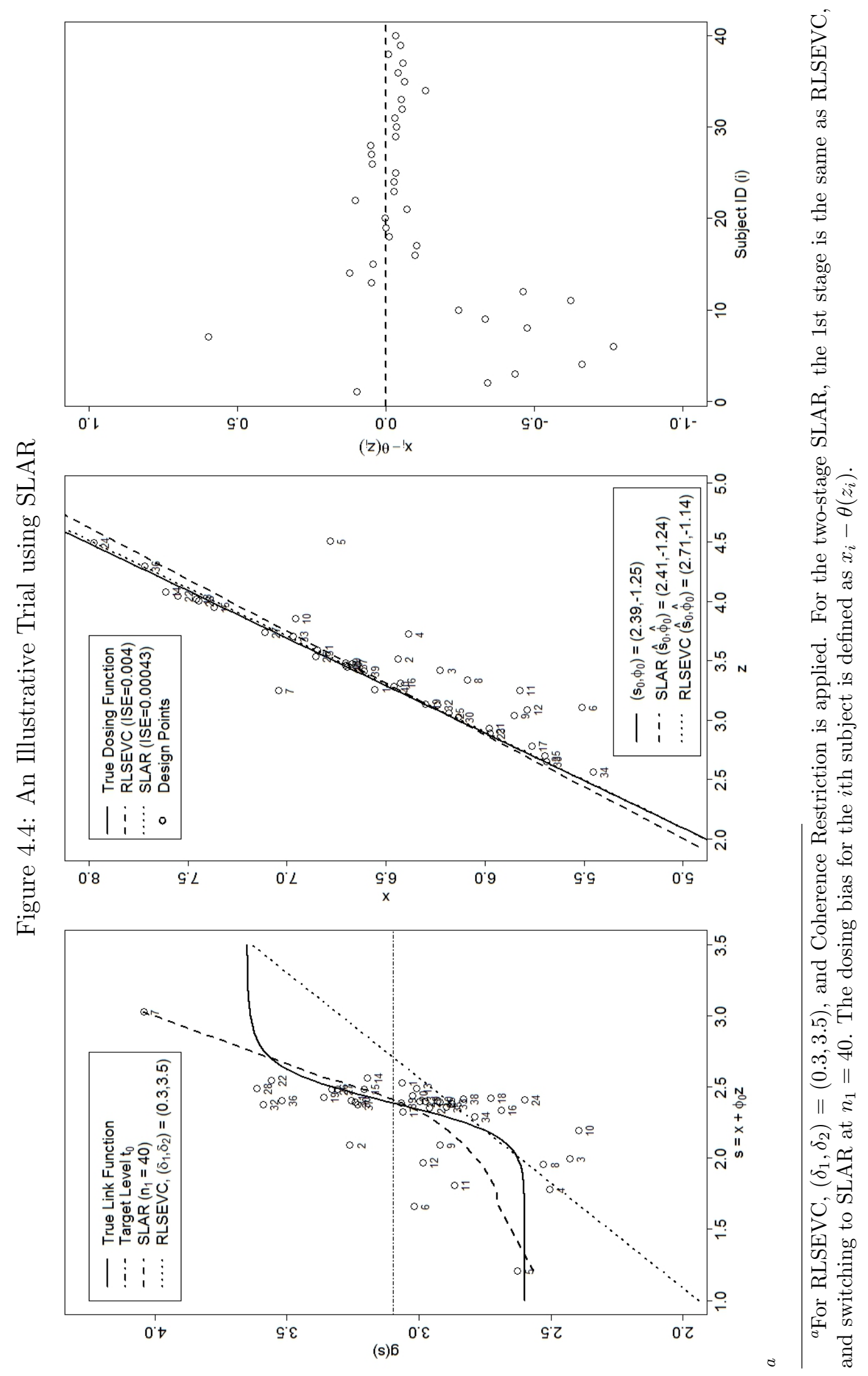


It largely corrects the location where the estimated curve crosses the target level from that $\left(\hat{\phi}_{n}=-1.14\right)$ obtained by RLSEVC. Due to the comparatively large variation of the noises and the tendency that the indexes concentrate around $s_{0}$, the shape of mean response curve estimated by SLAR is not very close to the true emax curve. But, the shape does not impact the estimation of $s_{0}$ indeed, as long as the estimated curve crosses the target level $t_{0}$ close to the correct location, because which actually determines the intercept $s_{0}$ of the true dosing function (4.8).

The plot in the middle of Figure 4.4 displays the true dosing function and estimated dosing functions by RLSEVC and SLAR, respectively. Although the estimated function by RLSEVC is already close (ISE $=0.004)$ to the true one, SLAR further improves the estimation ( ISE $=0.0004)$ so that the estimated function by SLAR is nearly identical to the true function. In addition, the right plot shows the sequential dosing biases of the simulated trial, which does no relation with SLAR, but indicates that the dosing accuracy of RLSEVC is actually acceptable even under a nonlinear model, in other words, RLSEVC is acceptably robust against link misspecification.

From this illustration, it can be found that the dosing cost and MISE of RLSEVC are actually acceptable under model misspecification, however, the semiparametric link-adapted recursion can even further improve the estimation precision of the model parameters and dosing function. In the subsequent section, a large-scale simulation study will further confirm this point.

\subsubsection{Design Comparison under Semiparametric Models}

In this section, a simulation study is presented to comprehensively compare the performance of the two-stage SLAR with the repeat least squares procedure under both the linear and semiparametric models. The goals of this simulation study include: demonstrating the advantage of SLAR over the repeated least squares procedure under semiparametric models, 
investigating the impact of the switching point $n_{1}$ on the performance of SLAR and attempt to identify out a recommended switching point, and investigating the robustness of the repeated least squares procedure to link misspecification. The criteria (dosing cost and ISE) assessing the design's performance are still the same as those defined in Section 3.1.

In the simulations, the first stage design uses both RLS and RLSEVC for comparison, where $\left(\delta_{1}, \delta_{2}\right)$ is chosen at $(0.5,2)$ and $(0.3,3.5)$. Due to the benefits of coherence restriction demonstrated in all the previous simulations, it will be applied to RLS and RLSEVC as a default. For the second stage of SLAR, the switching point $n_{1}$ is respectively selected at 25 , 30, 35 and 40. When $n_{1}=40$, the first stage design takes charge of dose assignments for the entire trial, while the second stage SLAR will be used to only re-estimate the dosing function at the end. In the comparison, RLS and RLSEVC will be regarded as special cases of the two-stage SLAR, where there is no second stage.

The comparison across designs and different choices of $n_{1}$ is conducted under four representative models, among which two are the linear models (the 1st and 6th scenario in Table 3.1 used in the simulation studies in Section 3.5), and the other two are respectively the $\mathrm{E}_{\max }$ link and power link models introduced at the end of Section 4.4.1. The reason for choosing the 1st and 6th scenarios as the linear models is that they typically represent the regular and irregular linear scenarios, which are discussed at the beginning of Section 3.5. Under each model, in total $N=5000$ trials with sample size $n=40$ will be simulated using each design. The simulation results under linear and semiparametric models are respectively summarized by Table 4.1 and 4.2 .

As shown by Table 4.1, the general pattern among the results under the linear models is that, the two-stage SLAR performs close to but slightly worse than the one-stage RLSEVC in terms of MISE, but remains nearly the same level of dosing costs. Although the estimation precision of SLAR is slightly worse than RLSEVC under the linear models, the difference in MISE is so small that is reasonable and acceptable. After all, if the linear model is the true 


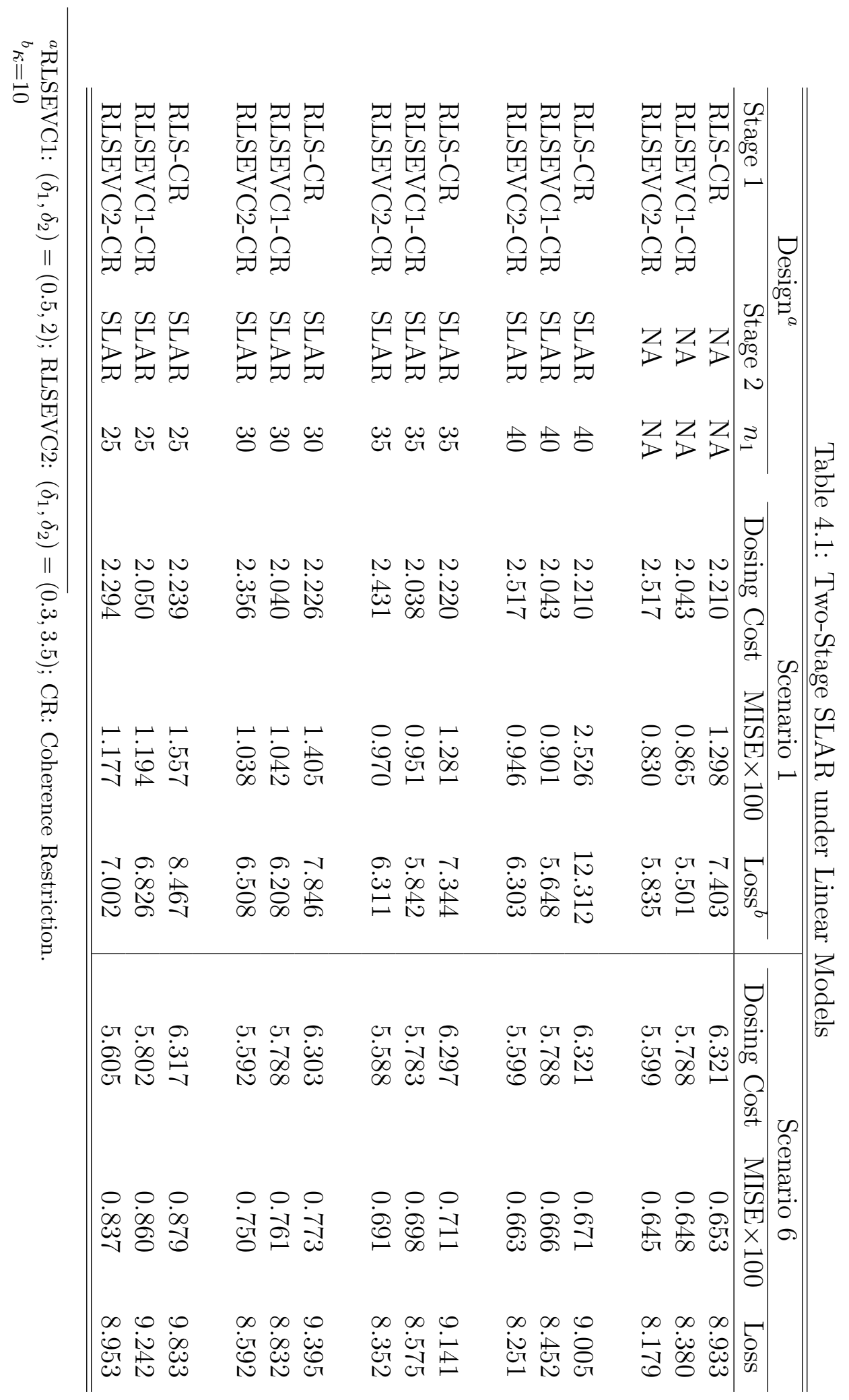


model, the parametric methods should be more precise than the semiparametric approaches, especially considering of the small sample size under our phase I trial settings. For example, under the 1st linear scenario, when the two-stage design is $\operatorname{RLSEVC}\left(\left(\delta_{1}, \delta_{2}\right)=(0.5,2)\right)$ connected with SLAR at $n_{1}=40$, it produces an MISE (0.901) that is only $4.2 \%$ larger than the MISE (0.865) of the corresponding RLSEVC.

Specifically, under the 1st linear scenario, RLSEVC with $\left(\delta_{1}, \delta_{2}\right)=(0.5,2)$ is the best design in the sense of minimizing the loss criterion; for the two-stage SLAR, as the switching point $n_{1}$ decreases from 40 to 25 , MISE gradually increases, while the dosing cost remains the same in general or even slowly decreases for RLSEVC with $\left(\delta_{1}, \delta_{2}\right)=(0.3,3.5)$. The decreasing pattern of MISE on $n_{1}$ indicates that, for SLAR, the switching point $n_{1}$ should not be too small (less than 30), otherwise, there would not be enough observations to ensure the precision of local linear regression and thus the precision of estimated dosing function.

In addition, it is also obvious that the estimation precision of SLAR relies on the first stage design. For each fixed $n_{1}$, by comparing MISE across the three different first stage designs, it can be found that the more precisely the first stage design performs in estimation, the more precise the estimated dosing function suggested by SLAR will be. Among the three, RLS would be the worst choice for the first stage, while RLSEVC with $\left(\delta_{1}, \delta_{2}\right)=(0.5,2)$ would be a better choice due to its smaller loss. The reason is because of the effect of eigenvalue constraint in spreading design points. The estimated indexes tend to concentrate around $s_{0}$, so the eigenvalue constraint can also help SLAR improve local linear fitting by slowing down the speed in which the indexes approach $s_{0}$. It would interesting to notice that, when using RLS as the first stage design and switching to SLAR at the end $\left(n_{1}=40\right)$, the MISE produced is even much larger than those corresponding to smaller $n_{1}$. The reason is because the effect of asymptotic singularity accumulated towards the end of some problematic trials and results in poor least squares estimation. When switching to SLAR at the end RLS, SLAR cannot correct the poor estimation but could produce even worse estimation due to 
the poor initial values. It again demonstrates that the performance of SLAR relies on the first stage design, and RLSEVC is a much reliable choice than RLS.

Under the 6th linear scenario, the similar patterns among dosing cost and MISE can be found, only except for that the difference across different $n_{1}$ is smaller due to the truncation on the estimated dosing function. The simulation results under the two linear models demonstrate that the two-stage SLAR with RLSEVC as the first stage does not sacrifice much precision to gain the robustness and generality. Considering of the small sample size at these cases, the semiparametric estimation is fairly accurate enough compared to the parametric methods.

As in Table 4.2, the simulation results under the semiparametric models show a general pattern that, the two-stage SLAR performs much better than the one-stage repeated least squares procedure in terms of MISE, meanwhile takes even smaller dosing costs. As $n_{1}$ decreases from 40 to 25, MISE of the two-stage SLAR reduces at first from 40 to 35, and then turns to increases gradually since 35 downwards to 25 ; while the dosing cost of the two-stage SLAR at $n_{1}=40$ reduces largely compared to the corresponding RLSEVC, and then further decreases slightly as $n_{1}$ decreases. For instance, under the Emax link model, when switching from $\operatorname{RLSEVC}\left(\left(\delta_{1}, \delta_{2}\right)=(0.5,2)\right)$ to SLAR at $n_{1}=35$, the resultant MISE (0.538) gets a 39.2\% reduction from the MISE (0.885) of RLSEVC; also for this example, the dosing cost (4.796) and loss (6.948) of the two-stage SLAR are respectively $3.4 \%$ and 18.3\% smaller than the cost (4.966) and loss (8.506) of RLSEVC.

Particularly, under the Emax link model, the pattern that MISE varies with $n_{1}$ indicates that, the switching point $n_{1}$ should not be smaller than 30 for the two-stage SLAR, and $n_{1}$ around 35 could be a good choice for stage-switch. Because of the local linear fit used by the second stage design, if the number of observations is small, less accurate nonparametric estimates would adversely affect the precision of estimated dosing function. In addition, the dependency of SLAR on the first stage design is still clear, such that the two-stage SLAR 


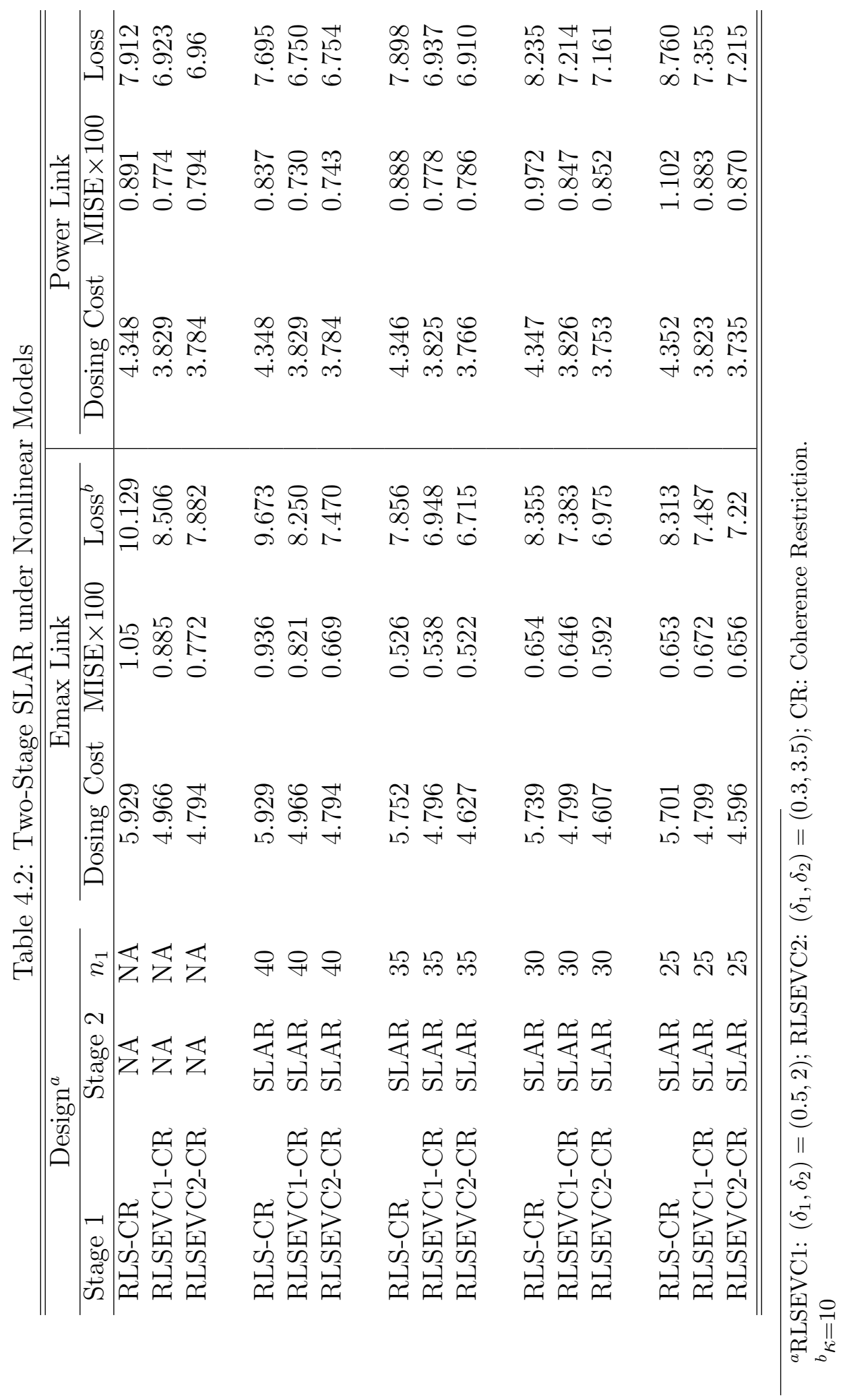


performs the worst when the first stage is RLS and produces much better dosing cost and MISE when the first stage is RLSEVC. According to the loss criterion, we suggest to use RLSEVC with $\left(\delta_{1}, \delta_{2}\right)=(0.3,3.5)$ as the first stage design.

Under the Power link model, the general pattern among the numerical results is similar, only except for the improvements from one-stage RLSEVC to two-stage SLAR are not as significant as those under the Emax link model. It is probably because the power link curve is closer to a linear model compared to the Emax link curve, and so the advantages of SLAR are not highlighted enough. Since the two-stage SLAR with $n_{1}=40$ produces the best MISE, it would be possible that an ideal switching point sits in between 40 and 35 . Therefore, an extra set of simulation using the two-stage SLAR with $n_{1}=38$ is conducted. The resultant dosing costs are 4.347, 3.828 and 3.776, meanwhile MISE's are respectively $0.855,0.746$ and 0.758, corresponding to the first stage being RLS, RLSEVC1 and RLSEVC2 as in Table 4.2. These results exactly sit in between those of $n_{1}=40$ and $n_{1}=35$, thus it should be better to switch to SLAR at the end of RLSEVC under the power link model.

Figure 4.5 presents the overall trend in which the dosing cost per each subject varies as a trial proceeds for the two-stage SLAR under linear and semiparametric models. The first stage design is RLSEVC with $\left(\delta_{1}, \delta_{2}\right)=(0.3,3.5)$, and the switching point $n_{1}$ is selected at 40, 35, 30 and 25. For $n_{1}=40$, since SLAR is only involved in re-estimation for the dosing function at the end of Stage 1, the dosing costs are actually the identical to those of RLSEVC. The upper three sub-figure plot the general trend over an entire trial under the 1st linear scenario, Emax link and power link models, while the lower three are the zoomed-in plots that focus on the later half trial, where the design is switched from RLSEVC to SLAR.

The general trend of the individual dosing cost is still similar to that displayed under linear models. The personal cost starts with increasing slightly at the first several subjects, then decreases dramatically around the 10th subject, and turns to approach 0 slowly in the later half trial. This trend demonstrates that the recommended dose sequence should be 

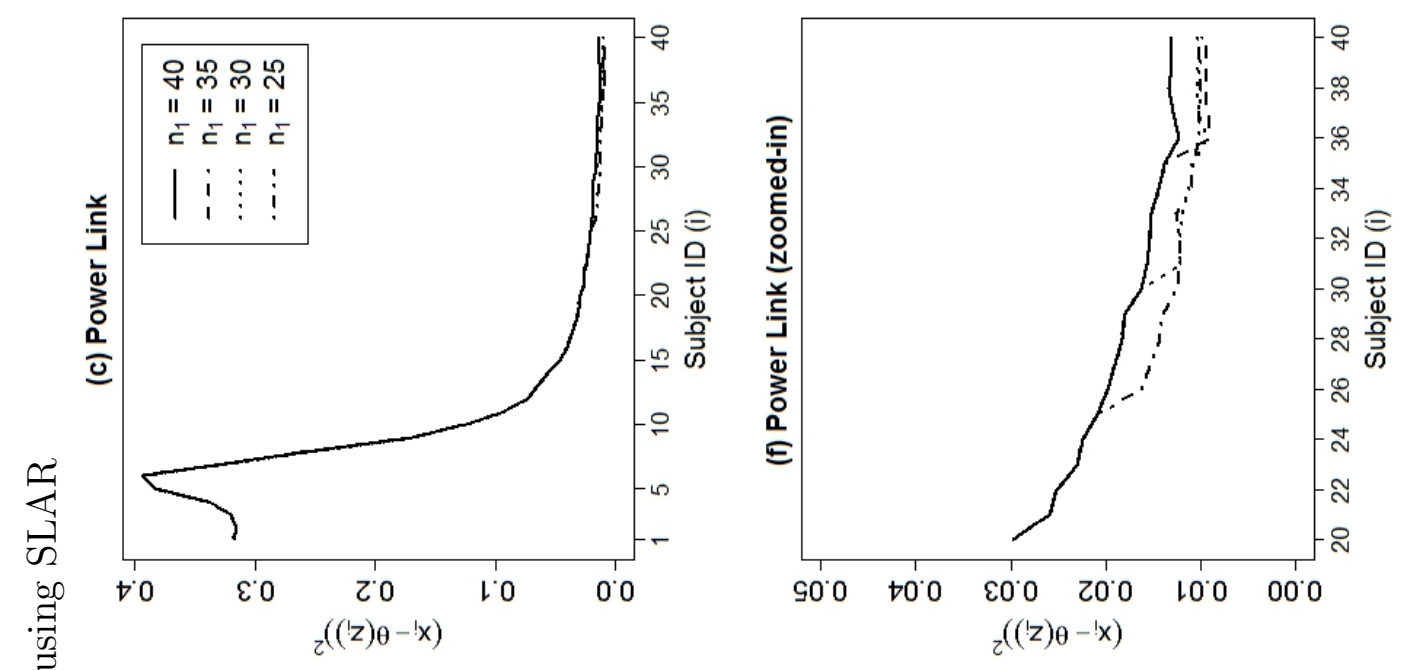

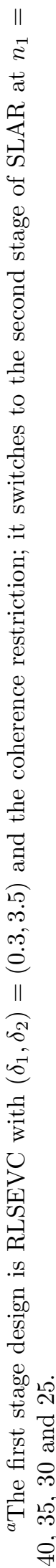


consistent, and the cost paid by the early subjects benefit those in later trial greatly.

From the zoomed-in plots, it would be interesting to find that, at each switching point smaller than 40, the individual dosing cost drops downwards clearly when switching from RLSEVC to SLAR. It indicates that switching from RLSEVC to SLAR can help further reduce the dosing cost. For example, under the Emax link model, the overall dosing cost per person of SLAR is further lowered down to 0.01 for the last 10 subjects in a trial. Considering of the small trial size and the complicated semiparametric recursion algorithms, the dosing accuracy is impressive indeed.

In summary, the simulation study demonstrates that, the two-stage semiparametric linkadapted recursion design is more robust to link misspecification than the repeated least squares procedure while trades very limited precision for the improved robustness. When the true model is semiparametric with an unknown link, the performance of two-stage SLAR is better than the repeated least squares procedure in terms of dosing cost and MISE, although RLSEVC already performs acceptably well under link misspecification. We advocate the use of RLSEVC with $\left(\delta_{1}, \delta_{2}\right)=(0.3,3.5)$ as the first stage design, meanwhile the switching point $n_{1}$ should be chosen close to $n$. For instance, $n_{1}$ could be chosen between 35 and 40 for $n=40$ at our case. In real-world practice, where the true model is never known, a conservative but reliable strategy would be, to use RLSEVC at the first stage design and, at its end, apply SLAR to re-estimate the dosing function. Based on the simulation results under both linear and semiparametric models, the loss corresponding to this design would be very close to the smallest loss among all.

\subsection{Theoretical Results}

The primary estimation interests within the procedure of SLAR are estimation of the direction parameter $\phi$ and unknown link function $g($.$) . In order to ensure the consistency of dose$ 
sequence (4.12) recommended by SLAR, it would be necessary that the recursive procedure results in consistent estimators for both $\phi_{0}$ and $s_{0}=g^{-1}\left(t_{0}\right)$.

Due to the iterative estimation manner of ISLS, the asymptotic properties should be decomposed into two parts in general. One part is that, given a consistent estimator of $\phi_{0}$, the isotonized local linear estimators (4.4) of the link function $g($.$) and its first order$ derivative $g^{\prime}($.$) is consistent. The other part is that, given consistent estimators of g($. and $g^{\prime}($.$) , the estimator (4.6) of \phi_{0}$ obtained by solving the pseudo score equation (4.5) is consistent. For simplicity, we temporarily only consider the cases without the isotonization. The major theoretical results are summarized by the following two conjectures.

Conjecture 1 Suppose that Condition 1.1 holds and $h=O\left(n^{-1 / 5}\right)$. Under the semiparametric link-adapted recursion design, if the local linear estimators (4.4) satisfy that,

$$
\operatorname{MSE}\left(\hat{g}_{n}(s)\right)=O\left(n^{-2 / 5}\right), \operatorname{MSE}\left(\hat{g}_{n}^{\prime}(s)\right)=O\left(n^{-1 / 5}\right)
$$

and

$$
\begin{aligned}
& \sup _{s \in I_{S}^{*}}\left|\hat{g}_{n}(s)-g(s)\right|=O\left(\frac{\sqrt{\log n}}{n^{2 / 5}}\right) \text { a.s., } \\
& \sup _{s \in I_{S}^{*}}\left|\hat{g}_{n}^{\prime}(s)-g^{\prime}(s)\right|=O\left(\frac{\sqrt{\log n}}{n^{1 / 5}}\right) \text { a.s., }
\end{aligned}
$$

where $\operatorname{MSE}\left(\hat{g}_{n}(s)\right)=E\left(\hat{g}_{n}(s)-g(s)\right)^{2}, \operatorname{MSE}\left(\hat{g}_{n}^{\prime}(s)\right) E\left(\hat{g}_{n}^{\prime}(s)-g^{\prime}(s)\right)^{2}$, and $I_{S}^{*}$ is a bounded closed interval in $I_{S}$ such that $s(\phi) \in I_{S}^{*}$ for any $\phi \in I_{\phi_{0}}^{*}$.

Then, the root sequence $\hat{\phi}_{n}$ (4.10) to the pseudo score equations (4.5) is weakly consistent for the true value $\phi_{0}$.

Conjecture 2 Suppose that Condition 1.1 through 1.4 hold. If the estimator $\hat{\phi}_{n}$ is weakly consistent for $\phi_{0}$, then, for any fixed $s \in I_{S}$, there are the following convergence rates for the 
local linear estimator:

$$
\begin{aligned}
\operatorname{Bias}\left(\hat{g}_{n}(s)\right) & =E\left(\hat{g}_{n}(s)-g(s)\right) \\
& =h^{2} g^{(2)}(s) \frac{\left(\mu_{K 2}^{2}-\mu_{K 1} \mu_{K 3}\right)}{2\left(\mu_{K 0} \mu_{K 2}-\mu_{K 1}^{2}\right)}+o\left(h^{2}\right), \\
\operatorname{Bias}\left(\hat{g}_{n}^{\prime}(s)\right) & =E\left(\hat{g}_{n}^{\prime}(s)-g^{\prime}(s)\right) \\
& =h^{2}\left(g^{(3)}(s)+3 g^{(2)}(s) f^{\prime}(s) / f(s)\right) \frac{\left(\mu_{K 0} \mu_{K 4}-\mu_{K 1} \mu_{K 3}\right)}{6\left(\mu_{K 0} \mu_{K 2}-\mu_{K 1}^{2}\right)}+o\left(h^{2}\right), \\
\operatorname{Var}\left(\hat{g}_{n}(s)\right) & =E\left(\hat{g}_{n}(s)-E\left(\hat{g}_{n}(s)\right)\right)^{2} \\
& =\frac{1}{n h} \frac{\sigma^{2}\left(\mu_{K 2}^{2} \nu_{K 0}-2 \mu_{K 1} \mu_{K 2} \nu_{K 1}+\mu_{K 1}^{2} \nu_{K 2}\right)}{f(s)\left(\mu_{K 0} \mu_{K 2}-\mu_{K 1}^{2}\right)^{2}}+o\left(\frac{1}{n h}\right) \\
\operatorname{Var}\left(\hat{g}_{n}^{\prime}(s)\right) & =E\left(\hat{g}_{n}^{\prime}(s)-E\left(\hat{g}_{n}^{\prime}(s)\right)\right)^{2} \\
& =\frac{1}{n h^{3}} \frac{\sigma^{2}\left(\mu_{K 1}^{2} \nu_{K 0}-2 \mu_{K 0} \mu_{K 1} \nu_{K 1}+\mu_{K 0}^{2} \nu_{K 2}\right)}{f(s)\left(\mu_{K 0} \mu_{K 2}-\mu_{K 1}^{2}\right)^{2}}+o\left(\frac{1}{n h^{3}}\right),
\end{aligned}
$$

where $\mu_{K j}=\int u^{j} K(u) d u$ for $j=0, \cdots, 4$, and $\nu_{K j}=\int u^{j} K^{2}(u) d u$ for $j=0,1,2$; and,

$$
\begin{aligned}
& \sup _{s \in I_{S}^{*}}\left|\hat{g}_{n}(s)-g(s)\right|=O\left(\left(\frac{\log n}{n h}\right)^{1 / 2}\right) \text { a.s., } \\
& \sup _{s \in I_{S}^{*}}\left|\hat{g}_{n}^{\prime}(s)-g^{\prime}(s)\right|=O\left(\left(\frac{\log n}{n h^{3}}\right)^{1 / 2}\right) \text { a.s., }
\end{aligned}
$$

where $I_{S}^{*}$ is a bounded closed interval in $I_{S}$ such that $s(\phi) \in I_{S}^{*}$ for any $\phi \in I_{\phi_{0}}^{*}$.

\section{Condition 1}

1. The unknown link function $g($.$) is non-decreasing and third time continuously differ-$ entiable within a bounded closed interval $I_{S} \subset(-\infty,+\infty)$, where $I_{S}$ is the domain of $g($.$) . Moreover, g($.$) is strictly increasing among a neighborhood of s_{0}=g^{-1}\left(t_{0}\right)$.

2. The kernel function $K($.$) is assumed to a symmetric continuous probability density$ function with bounded support. $K\left(\right.$.) has up to 4 th finite moments, meanwhile $K^{2}()$. 
has up to 2nd finite moments.

3. The probability density function $f(s)$ of the index $s=x+\phi z, f(s)>0$ for any $s \in I_{S}$ and, $f(s)$ and $f^{\prime}(s)$ are continuous in $I_{S}$.

4. The bandwidth $h \rightarrow 0$, $n h \rightarrow+\infty$ and $n h^{3} \rightarrow+\infty$, as $n \rightarrow+\infty$.

\section{Comments 1}

1. Given the asymptotic bias (4.22) and variance (4.24), it would be straightforward to derive out the asymptotic mean squared error (MSE) of the local linear estimate of $g(s)$, such that

$$
\begin{aligned}
& \operatorname{MSE}\left(\hat{g}_{n}(s)\right)=E\left(\hat{g}_{n}(s)-g(s)\right)^{2} \\
= & \operatorname{Bias}\left(\hat{g}_{n}(s)\right)^{2}+\operatorname{Var}\left(\hat{g}_{n}(s)\right) \\
\sim & \frac{h^{4} g^{(2)}(s)^{2}\left(\mu_{K 2}^{2}-\mu_{K 1} \mu_{K 3}\right)^{2}}{4\left(\mu_{K 0} \mu_{K 2}-\mu_{K 1}^{2}\right)^{2}}+\frac{\sigma^{2}\left(\mu_{K 2}^{2} \nu_{K 0}-2 \mu_{K 1} \mu_{K 2} \nu_{K 1}+\mu_{K 1}^{2} \nu_{K 2}\right)}{n h f(s)\left(\mu_{K 0} \mu_{K 2}-\mu_{K 1}^{2}\right)^{2}} \\
\rightarrow & 0, \quad \text { as } n \rightarrow \infty
\end{aligned}
$$

Therefore, by Chebyshev's inequality, it can be obtained that the local linear estimator $\hat{g}_{n}(s)$ is weakly consistent for $g(s)$; and, likewise for the week consistency of $\hat{g}_{n}^{\prime}(s)$.

2. By minimizing the $\operatorname{MSE}\left(\hat{g}_{n}(s)\right)$ (4.28) with respect to the bandwidth $h$, we can derive out the optimal rate for the bandwidth.

$$
\begin{aligned}
& \frac{\partial \operatorname{MSE}\left(\hat{g}_{n}(s)\right)}{\partial h}=0 \\
\Rightarrow & \frac{\partial}{\partial h}\left\{\frac{h^{4} g^{(2)}(s)^{2}\left(\mu_{K 2}^{2}-\mu_{K 1} \mu_{K 3}\right)^{2}}{4\left(\mu_{K 0} \mu_{K 2}-\mu_{K 1}^{2}\right)^{2}}+\frac{\sigma^{2}\left(\mu_{K 2}^{2} \nu_{K 0}-2 \mu_{K 1} \mu_{K 2} \nu_{K 1}+\mu_{K 1}^{2} \nu_{K 2}\right)}{n h f(s)\left(\mu_{K 0} \mu_{K 2}-\mu_{K 1}^{2}\right)^{2}}\right\}=0 \\
\Rightarrow & \frac{h^{3} g^{(2)}(s)^{2}\left(\mu_{K 2}^{2}-\mu_{K 1} \mu_{K 3}\right)^{2}}{\left(\mu_{K 0} \mu_{K 2}-\mu_{K 1}^{2}\right)^{2}}-\frac{\sigma^{2}\left(\mu_{K 2}^{2} \nu_{K 0}-2 \mu_{K 1} \mu_{K 2} \nu_{K 1}+\mu_{K 1}^{2} \nu_{K 2}\right)}{n h^{2} f(s)\left(\mu_{K 0} \mu_{K 2}-\mu_{K 1}^{2}\right)^{2}}=0 \\
\Rightarrow & h_{o p t}=\left\{\frac{1}{n} \frac{\sigma^{2}\left(\mu_{K 2}^{2} \nu_{K 0}-2 \mu_{K 1} \mu_{K 2} \nu_{K 1}+\mu_{K 1}^{2} \nu_{K 2}\right)}{f(s) g^{(2)}(s)^{2}\left(\mu_{K 2}^{2}-\mu_{K 1} \mu_{K 3}\right)^{2}}\right\}^{-1 / 5}
\end{aligned}
$$




$$
\Rightarrow h_{o p t}=O\left(n^{-1 / 5}\right)
$$

For Conjecture 1, the goal is to show that a sequence of roots to the following pseudo score equations is weakly consistent for the true value:

$$
\hat{\mathcal{S}}_{n}(\phi)=\frac{1}{n} \sum_{k=1}^{n} z_{k} \hat{g}_{n}^{\prime}\left(s_{k}(\phi)\right)\left\{y_{k}-\hat{g}_{n}\left(s_{k}(\phi)\right)\right\}=0, \quad n=n_{1}, n_{1}+1, \cdots
$$

where $s_{k}(\phi)=x_{k}+\phi z_{k}$ for $k=1, \cdots, n$. Thus, the asymptotic theory of estimating equation estimator would be the natural technique to utilize. However, most existing theorems (for example Yuan and Jennrich [1998]) that establish consistency of the estimating equation estimator assume independence among the observations, or at least independent observations between subjects for the cases with repeated measurements. For the semiparametric link-adapted recursion design, each dose $x_{i}$ (4.13) depends on all the previous data $\left\{\left(y_{k}, x_{k}, z_{k}\right)\right\}_{k=1}^{i-1}$ via the recursive estimation of $s_{0}$ and $\phi_{0}$. Furthermore, both the estimates, $\hat{s}_{0 i}$ and $\hat{\phi}_{i}$, do not have explicit analytic forms, because they are obtained by using numerical algorithms. These bring with us the major difficulties to determine which type of stochastic processes the dose sequence belongs to, and then investigate the asymptotic behavior of the stochastic process.

Fortunately, recent development in the theory of estimating equations with stochastic processes provides us with a promising method to establish consistency of the root sequence to the estimating equations (4.30). Kessler et al. [2012] introduce the asymptotic theorems of estimating functions for martingales and diffusion-type processes in the first chapter of their book. In this chapter, most often, the estimating equations are for specific types of stochastic processes, for example a martingale, Markov chain, or stationary process with certain mixing conditions. In Section 1.10, the authors propose a general theorem for the 
weak consistency of estimating equation estimator for a general stochastic process. By using the notations in our context, the theorem can be stated as follows.

Lemma 2 (Theorem 1.10.2 of Kessler et al. [2012]) The data $\left\{\left(y_{k}, x_{k}, z_{k}\right)\right\}_{k=1}^{n}$ are observations from a stochastic process, and the estimator $\hat{\phi}_{n}$ obtained by solving the estimating equation $\hat{\mathcal{S}}_{n}\left(\phi_{0}\right)=0$ is an arbitrary function of the data with value in $I_{\phi}$. Suppose the existence of a true parameter $\phi_{0} \in \operatorname{int} I_{\phi}$ (the interior of $I_{\phi}$ ), a bounded neighborhood $I_{\phi_{0}}^{*}$ of $\phi_{0}$ within $I_{\phi}$, and a (random) function $\mathcal{S}_{0}^{*}($.$) defined on I_{\phi_{0}}^{*}$, such that the following conditions hold:

i)

$$
\hat{\mathcal{S}}_{n}\left(\phi_{0}\right) \stackrel{P}{\rightarrow} 0 \text { as } n \rightarrow \infty
$$

ii) $\hat{\mathcal{S}}_{n}(\phi)$ is continuously differentiable on $I_{\phi_{0}}^{*}$ for all $n$, and

$$
\sup _{\phi \in I_{\phi_{0}}^{*}}\left|\frac{d \hat{\mathcal{S}}_{n}(\phi)}{d \phi}-\mathcal{S}_{0}^{*}(\phi)\right| \stackrel{P}{\rightarrow} 0
$$

where $\mathcal{S}_{0}^{*}\left(\phi_{0}\right) \neq 0$ a.s..

Then, a sequence $\hat{\phi}_{n}$ that is the zero-root to estimating function $\hat{\mathcal{S}}_{n}(\phi)$ is weakly consistent for $\phi_{0}$. Moreover, this root sequence is eventually unique, in the sense that, for any other root sequence $\tilde{\phi}_{n}$ to $\hat{\mathcal{S}}_{n}(\phi)=0$ that is consistent for $\phi_{0}, P\left(\hat{\phi}_{n} \neq \tilde{\phi}_{n}\right) \rightarrow 0$, as $n \rightarrow \infty$.

Based on Lemma 2, we make the Conjecture 1 on the consistency of the root sequence to the pseudo score equation (4.30), and also have the following sketch of proof for it.

Proof Sketch (Conjecture 1) In order to verify the condition (4.31), we at first decompose $\hat{\mathcal{S}}_{n}(\phi)$ into 4 parts as follows.

$$
\hat{\mathcal{S}}_{n}(\phi)=\frac{1}{n} \sum_{k=1}^{n} z_{k} \hat{g}_{n}^{\prime}\left(s_{k}(\phi)\right)\left\{y_{k}-\hat{g}_{n}\left(s_{k}(\phi)\right)\right\}
$$




$$
\begin{aligned}
= & \frac{1}{n} \sum_{k=1}^{n} z_{k}\left\{g^{\prime}\left(s_{k}(\phi)\right)+\hat{g}_{n}^{\prime}\left(s_{k}(\phi)\right)-g^{\prime}\left(s_{k}(\phi)\right)\right\}\left\{y_{k}-g\left(s_{k}(\phi)\right)-\left[\hat{g}_{n}\left(s_{k}(\phi)\right)-g\left(s_{k}(\phi)\right)\right]\right\} \\
= & \frac{1}{n} \sum_{k=1}^{n} z_{k} g^{\prime}\left(s_{k}(\phi)\right)\left(y_{k}-g\left(s_{k}(\phi)\right)\right)-\frac{1}{n} \sum_{k=1}^{n} z_{k} g^{\prime}\left(s_{k}(\phi)\right)\left(\hat{g}_{n}\left(s_{k}(\phi)\right)-g\left(s_{k}(\phi)\right)\right) \\
& +\frac{1}{n} \sum_{k=1}^{n} z_{k}\left(\hat{g}_{n}^{\prime}\left(s_{k}(\phi)\right)-g^{\prime}\left(s_{k}(\phi)\right)\right)\left(y_{k}-g\left(s_{k}(\phi)\right)\right) \\
& -\frac{1}{n} \sum_{k=1}^{n} z_{k}\left(\hat{g}_{n}^{\prime}\left(s_{k}(\phi)\right)-g^{\prime}\left(s_{k}(\phi)\right)\right)\left(\hat{g}_{n}\left(s_{k}(\phi)\right)-g\left(s_{k}(\phi)\right)\right) \\
= & \mathcal{S}_{n 1}(\phi)-\hat{\mathcal{S}}_{n 2}(\phi)+\hat{\mathcal{S}}_{n 3}(\phi)-\hat{\mathcal{S}}_{n 4}(\phi),
\end{aligned}
$$

where $\mathcal{S}_{n 1}\left(\phi_{0}\right)=\frac{1}{n} \sum_{k=1}^{n} z_{k} g^{\prime}\left(s_{k}\left(\phi_{0}\right)\right)\left(y_{k}-g\left(s_{k}\left(\phi_{0}\right)\right)\right), \hat{\mathcal{S}}_{n 2}\left(\phi_{0}\right)=\frac{1}{n} \sum_{k=1}^{n} z_{k} g^{\prime}\left(s_{k}(\phi)\right)$ $\left(\hat{g}_{n}\left(s_{k}(\phi)\right)-g\left(s_{k}(\phi)\right)\right), \hat{\mathcal{S}}_{n 3}\left(\phi_{0}\right)=\frac{1}{n} \sum_{k=1}^{n} z_{k}\left(\hat{g}_{n}^{\prime}\left(s_{k}(\phi)\right)-g^{\prime}\left(s_{k}(\phi)\right)\right)\left(y_{k}-g\left(s_{k}(\phi)\right)\right)$, and $\hat{\mathcal{S}}_{n 4}\left(\phi_{0}\right)=\frac{1}{n} \sum_{k=1}^{n} z_{k}\left(\hat{g}_{n}^{\prime}\left(s_{k}(\phi)\right)-g^{\prime}\left(s_{k}(\phi)\right)\right)\left(\hat{g}_{n}\left(s_{k}(\phi)\right)-g\left(s_{k}(\phi)\right)\right)$, Then, the condition (4.31) would hold if we could respectively show that $\mathcal{S}_{n 1}(\phi), \hat{\mathcal{S}}_{n 2}(\phi), \hat{\mathcal{S}}_{n 3}(\phi)$ and $\hat{\mathcal{S}}_{n 4}(\phi)$ converges to 0 in probability.

$$
\mathcal{S}_{n 1}\left(\phi_{0}\right)=\frac{1}{n} \sum_{k=1}^{n} z_{k} g^{\prime}\left(s_{k}\left(\phi_{0}\right)\right)\left(y_{k}-g\left(s_{k}\left(\phi_{0}\right)\right)\right)=\frac{1}{n} \sum_{k=1}^{n} z_{k} \epsilon_{k} g^{\prime}\left(s_{k}\left(\phi_{0}\right)\right) \text {, by the model (4.2). }
$$

If we could derive out that $\lim _{n \rightarrow \infty} s_{n}\left(\phi_{0}\right)=s_{\infty}$ a.s. for some $s_{\infty} \in I_{S}$, then $g^{\prime}\left(s_{n}\left(\phi_{0}\right)\right) \stackrel{\text { a.s. }}{\longrightarrow}$ $g^{\prime}\left(s_{\infty}\right)$ by the Continuous Mapping theorem. In addition, $g(s)$ is strictly increasing at $s_{\infty}$ and $g^{\prime}(s)$ is continuously differentiable on a bounded closed interval $I_{S}$, thus $g^{\prime}\left(s_{\infty}\right)>0$ and is bounded by some positive constant $B_{g^{\prime}}$.

Since $\left\{z_{k}\right\}$ and $\left\{\epsilon_{k}\right\}$ are independent with each other and both normally distributed with $E\left(z_{1}\right)=\mu_{z}<\infty$ and $E\left(\epsilon_{1}\right)=0$, by the Strong Law of Large Numbers (Theorem B on Page 27 of Serfling [1980]),

$$
\frac{1}{n} \sum_{k=1}^{n} z_{k} \epsilon_{k} g^{\prime}\left(s_{\infty}\right) \stackrel{\text { a.s. }}{\longrightarrow} 0, \quad \text { as } n \rightarrow \infty .
$$

Then, by showing that $\frac{1}{n} \sum_{k=1}^{n} z_{k} \epsilon_{k}\left\{g^{\prime}\left(s_{k}\left(\phi_{0}\right)\right)-g^{\prime}\left(s_{\infty}\right)\right\} \stackrel{\text { a.s. }}{\longrightarrow} 0$, it can be shown that 
$\mathcal{S}_{n 1}\left(\phi_{0}\right) \stackrel{\text { a.s. }}{\longrightarrow} 0$, as $n \rightarrow \infty$.

For any $\varepsilon>0$,

$$
\begin{aligned}
& P\left\{\left|\hat{\mathcal{S}}_{n 2}\left(\phi_{0}\right)\right|>\varepsilon\right\} \leq \frac{E\left|\hat{\mathcal{S}}_{n 2}\left(\phi_{0}\right)\right|}{\varepsilon} \quad \text { by Markov Inequality } \\
& =\frac{1}{n \varepsilon} E\left|\sum_{k=1}^{n} z_{k} g^{\prime}\left(s_{k}\left(\phi_{0}\right)\right)\left(\hat{g}_{n}\left(s_{k}\left(\phi_{0}\right)\right)-g\left(s_{k}\left(\phi_{0}\right)\right)\right)\right| \\
& \leq \frac{1}{n \varepsilon} \sum_{k=1}^{n} E\left\{\left|z_{k} g^{\prime}\left(s_{k}\left(\phi_{0}\right)\right)\right|\left|\hat{g}_{n}\left(s_{k}\left(\phi_{0}\right)\right)-g\left(s_{k}\left(\phi_{0}\right)\right)\right|\right\} \\
& \leq \frac{1}{n \varepsilon} \sum_{k=1}^{n}\left\{E\left(z_{k} g^{\prime}\left(s_{k}\left(\phi_{0}\right)\right)\right)^{2}\right\}^{1 / 2}\left\{E\left(\hat{g}_{n}\left(s_{k}\left(\phi_{0}\right)\right)-g\left(s_{k}\left(\phi_{0}\right)\right)\right)^{2}\right\}^{1 / 2} \text { by Holder Inequality } \\
& \leq \frac{B_{g^{\prime}}^{2}}{n \varepsilon} \sum_{k=1}^{n}\left(\operatorname{Var}\left(z_{k}\right)+\left(E z_{k}\right)^{2}\right)^{1 / 2}\left\{\operatorname{MSE}\left(\hat{g}_{n}\left(s_{k}\left(\phi_{0}\right)\right)\right)\right\}^{1 / 2}\left|g^{\prime}(s)\right| \text { is bounded by } B_{g^{\prime}} \text { on } I_{S} \\
& =\frac{B_{g^{\prime}}^{2}}{n \varepsilon} n \sqrt{\sigma_{z}^{2}+\mu_{z}^{2}} O\left(n^{-2 / 5}\right) \\
& =O\left(n^{-2 / 5}\right) \rightarrow 0, \quad \text { as } n \rightarrow \infty .
\end{aligned}
$$

Similarly, it can be shown that $P\left\{\left|\hat{\mathcal{S}}_{n 3}\left(\phi_{0}\right)\right|>\varepsilon\right\} \rightarrow 0$ and $P\left\{\left|\hat{\mathcal{S}}_{n 4}\left(\phi_{0}\right)\right|>\varepsilon\right\} \rightarrow 0$. Therefore, combining $\mathcal{S}_{n 1}\left(\phi_{0}\right)=o(1)$ a.s. and $\hat{\mathcal{S}}_{n i}\left(\phi_{0}\right)=o_{p}(1)$ for $i=2,3$, 4 , then there is

$$
\hat{\mathcal{S}}_{n}\left(\phi_{0}\right)=\mathcal{S}_{n 1}\left(\phi_{0}\right)-\hat{\mathcal{S}}_{n 2}\left(\phi_{0}\right)+\hat{\mathcal{S}}_{n 3}\left(\phi_{0}\right)-\hat{\mathcal{S}}_{n 4}\left(\phi_{0}\right) \stackrel{\mathrm{P}}{\rightarrow} 0, \quad \text { as } n \rightarrow \infty \text {. }
$$

In order to verify the condition $(4.32)$, we at first decompose $\frac{d \hat{\mathcal{S}}_{n}(\phi)}{d \phi}$ into eight parts as follows.

$$
\begin{aligned}
& \frac{d \hat{\mathcal{S}}_{n}(\phi)}{d \phi}=\frac{1}{n} \sum_{k=1}^{n}\left\{z_{k}\left[y_{k}-\hat{g}_{n}\left(s_{k}(\phi)\right)\right] \frac{d \hat{g}_{n}^{\prime}\left(s_{k}(\phi)\right)}{d \phi}-z_{k} \hat{g}_{n}^{\prime}\left(s_{k}(\phi)\right) \frac{d \hat{g}_{n}\left(s_{k}(\phi)\right)}{d \phi}\right\} \\
& =\frac{1}{n} \sum_{k=1}^{n}\left\{z_{k}\left[y_{k}-g\left(s_{k}(\phi)\right)-\hat{g}_{n}\left(s_{k}(\phi)\right)+g\left(s_{k}(\phi)\right)\right] \frac{d}{d \phi}\left(\hat{g}_{n}^{\prime}\left(s_{k}(\phi)\right)-g^{\prime}\left(s_{k}(\phi)\right)+g^{\prime}\left(s_{k}(\phi)\right)\right)\right. \\
& \left.\quad-z_{k}\left(\hat{g}_{n}^{\prime}\left(s_{k}(\phi)\right)-g^{\prime}\left(s_{k}(\phi)\right)+g^{\prime}\left(s_{k}(\phi)\right)\right) \frac{d}{d \phi}\left(\hat{g}_{n}\left(s_{k}(\phi)\right)-g\left(s_{k}(\phi)\right)+g\left(s_{k}(\phi)\right)\right)\right\}
\end{aligned}
$$




$$
\begin{aligned}
= & \frac{1}{n} \sum_{k=1}^{n} z_{k} g^{(2)}\left(s_{k}(\phi)\right)\left(y_{k}-g\left(s_{k}(\phi)\right)\right) \\
& +\frac{1}{n} \sum_{k=1}^{n} z_{k}\left(y_{k}-g\left(s_{k}(\phi)\right)\right) \frac{d}{d \phi}\left(\hat{g}_{n}^{\prime}\left(s_{k}(\phi)\right)-g^{\prime}\left(s_{k}(\phi)\right)\right) \\
& -\frac{1}{n} \sum_{k=1}^{n} z_{k} g^{(2)}\left(s_{k}(\phi)\right)\left(\hat{g}_{n}\left(s_{k}(\phi)\right)-g\left(s_{k}(\phi)\right)\right) \\
& -\frac{1}{n} \sum_{k=1}^{n} z_{k}\left(\hat{g}_{n}\left(s_{k}(\phi)\right)-g\left(s_{k}(\phi)\right)\right) \frac{d}{d \phi}\left(\hat{g}_{n}^{\prime}\left(s_{k}(\phi)\right)-g^{\prime}\left(s_{k}(\phi)\right)\right) \\
& -\frac{1}{n} \sum_{k=1}^{n} z_{k} g^{(2)}\left(s_{k}(\phi)\right)\left(\hat{g}_{n}^{\prime}\left(s_{k}(\phi)\right)-g^{\prime}\left(s_{k}(\phi)\right)\right) \\
& -\frac{1}{n} \sum_{k=1}^{n} z_{k}\left(\hat{g}_{n}^{\prime}\left(s_{k}(\phi)\right)-g^{\prime}\left(s_{k}(\phi)\right)\right) \frac{d}{d \phi}\left(\hat{g}_{n}\left(s_{k}(\phi)\right)-g\left(s_{k}(\phi)\right)\right) \\
& -\frac{1}{n} \sum_{k=1}^{n} z_{k} g^{\prime}\left(s_{k}(\phi)\right) \frac{d}{d \phi}\left(\hat{g}_{n}\left(s_{k}(\phi)\right)-g\left(s_{k}(\phi)\right)\right)-\frac{1}{n} \sum_{k=1}^{n} z_{k} g^{\prime}\left(s_{k}(\phi)\right)^{2} \\
= & \mathcal{S}_{n 1}^{*}(\phi)+\hat{\mathcal{S}}_{n 2}^{*}(\phi)-\hat{\mathcal{S}}_{n 3}^{*}(\phi)-\hat{\mathcal{S}}_{n 4}^{*}(\phi)-\hat{\mathcal{S}}_{n 5}^{*}(\phi)-\hat{\mathcal{S}}_{n 6}^{*}(\phi)-\hat{\mathcal{S}}_{n 7}^{*}(\phi)-\mathcal{S}_{n 8}^{*}(\phi) .
\end{aligned}
$$

We define that $\mathcal{S}_{0}^{*}(\phi)=\mathcal{S}_{\infty 1}^{*}(\phi)-\mathcal{S}_{\infty 8}^{*}(\phi)$ for $\phi \in I_{\phi}$, where $\mathcal{S}_{\infty 1}^{*}(\phi)=\lim _{n \rightarrow \infty} \mathcal{S}_{n 1}^{*}(\phi)$ and $\mathcal{S}_{\infty 8}^{*}(\phi)=\lim _{n \rightarrow \infty} \mathcal{S}_{n 8}^{*}(\phi)$. Then, to verify the condition (4.32), we need to show that

$$
\sup _{\phi \in I_{\phi_{0}}^{*}}\left|\hat{\mathcal{S}}_{n j}^{*}(\phi)\right| \stackrel{P}{\rightarrow} 0, \quad \text { for } j=2, \cdots, 7,
$$

and derive out the limits of $\mathcal{S}_{n 1}^{*}(\phi)$ and $\mathcal{S}_{n 8}^{*}(\phi)$ respectively. With the conditions (4.20) and (4.21), the result (4.33) can be verified by applying the same technique as used in showing $\hat{\mathcal{S}}_{n 2}\left(\phi_{0}\right) \stackrel{P}{\rightarrow} 0$.

The uniform convergence condition (4.32) is common for most two-stage semiparametric estimation methods, where the second stage depends on the first stage of nonparametric estimators. For example, the Theorem 3 of Yuan and Jennrich [1998] also requires that the estimating functions are continuous and $\sup _{\phi \in I_{\phi_{0}}^{*}}\left|\hat{\mathcal{S}}_{n}(\phi)-\mathcal{S}_{0}(\phi)\right| \stackrel{P}{\rightarrow} 0$ for some limit function $\mathcal{S}_{0}($.$) . In Lemma 2$ for stochastic processes, the uniform convergence condition is 
stronger than that of independent random variable case, that is, the condition (4.32) can imply that $\sup _{\phi \in I_{\phi_{0}}^{*}}\left|\hat{\mathcal{S}}_{n}(\phi)-\mathcal{S}_{0}(\phi)\right| \stackrel{P}{\rightarrow} 0$ for a bounded neighborhood $I_{\phi_{0}}^{*}$. The condition (4.31) would requires the consistency of local linear estimators (4.4) along with their corresponding point-wise convergence rates. Meanwhile, the condition (4.32) would be more mathematically challenging to verify, since it requires the uniform convergence rate of the local linear estimator.

It is worth to point out that, given the MSE convergence rates (4.19) and the uniform convergence rates (4.20) and (4.21), the challenging parts in showing the Conjecture 1 under the direction of Lemma 2 is the derivation of limits of $\mathcal{S}_{n 1}(\phi), \mathcal{S}_{n 1}^{*}(\phi)$ and $\mathcal{S}_{n 8}^{*}(\phi)$. And, the calculation of these limits depends on the type of stochastic processes that the dose sequence $x_{n}$ recommended by SLAR belongs to, as corresponding version of Law of Large Numbers should be used according the type of specific processes.

However, the most mathematically challenging part is actually how to obtain the aforementioned convergence rates for the local linear estimator with the dependent data collected under SLAR, that is, the Conjecture 2. Similar to Theorem 3.1 of Fan and Gijbels [1996], we conjecture the convergence rates for the asymptotic bias and variance of the local linear estimators (4.4) under SLAR; and, in the light of Stone [1982], we also conjecture the uniform convergence rates for the local linear estimators under SLAR.

Proof Sketch (Conjecture 2) Given a consistent $\hat{\phi}_{n}$ based on the observations at stage $n$ in SLAR, the local linear fit is applied to $\left(\hat{s}_{1}^{(n)}, y_{1}\right), \cdots,\left(\hat{s}_{n}^{(n)}, y_{n}\right)$, where $\hat{s}_{k}^{(n)}=x_{k}+\hat{\phi}_{n} z_{k}$ for $k=1, \cdots, n$ and obtain the point-wise estimates of link function and its first order derivative at any fixed $s \in I_{S}$.

$$
\begin{aligned}
\left(\hat{g}_{n}(s), \hat{g}_{n}^{\prime}(s)\right)^{\prime} & =\left(W_{n}^{\prime}(s) \Omega_{n}(s) W_{n}(s)\right)^{-1} W_{n}^{\prime}(s) \Omega_{n}(s) Y_{n} \\
& =\left(\begin{array}{ll}
V_{n 11} & V_{n 12} \\
V_{n 12} & V_{n 22}
\end{array}\right)^{-1}\left(\begin{array}{c}
U_{n 1} \\
U_{n 2}
\end{array}\right)
\end{aligned}
$$




$$
=\left(\begin{array}{c}
\frac{V_{n 22} U_{n 1}-V_{n 12} U_{n 2}}{V_{n 11} V_{n 22}-V_{n 12}^{2}} \\
\frac{V_{n 11} U_{n 2}-V_{n 12} U_{n 1}}{V_{n 11} V_{n 22}-V_{n 12}^{2}}
\end{array}\right)
$$

where $V_{n 11}=\frac{1}{n} \sum_{k=1}^{n} K_{h}\left(\hat{s}_{k}^{(n)}-s\right), V_{n 12}=\frac{1}{n} \sum_{k=1}^{n}\left(\hat{s}_{k}^{(n)}-s\right) K_{h}\left(\hat{s}_{k}^{(n)}-s\right)$, $V_{n 22}=\frac{1}{n} \sum_{k=1}^{n}\left(\hat{s}_{k}^{(n)}-s\right)^{2} K_{h}\left(\hat{s}_{k}^{(n)}-s\right), U_{n 1}=\frac{1}{n} \sum_{k=1}^{n} y_{k} K_{h}\left(\hat{s}_{k}^{(n)}-s\right)$, $U_{n 2}=\frac{1}{n} \sum_{k=1}^{n} y_{k}\left(\hat{s}_{k}^{(n)}-s\right) K_{h}\left(\hat{s}_{k}^{(n)}-s\right), W_{n}(s)=\left(\begin{array}{cc}1 & \hat{s}_{1}^{(n)}-s \\ \vdots & \vdots \\ 1 & \hat{s}_{n}^{(n)}-s\end{array}\right)$, and $\Omega_{n}(s)=\left(\begin{array}{ccc}K_{h}\left(\hat{s}_{1}^{(n)}-s\right) & \cdots & 0 \\ \vdots & \ddots & \vdots \\ 0 & \cdots & K_{h}\left(\hat{s}_{n}^{(n)}-s\right)\end{array}\right)$.

In fact, under the cases with independent and identically distributed data, the major techniques used in deriving the asymptotic bias and variance of local linear estimator typically include the Taylor expansion, approximation to the sums such as $V_{n i j}$ and $U_{n i j}$, and the asymptotic theorems that deal with the triangular arrays. For instance, when calculating the asymptotic bias and variance for either $\hat{g}_{n}(s)$ or $\hat{g}_{n}^{\prime}(s)$, the following technique is used frequently under the independence cases.

$$
\begin{aligned}
& E\left(\frac{1}{n} \sum_{k=1}^{n}\left(s_{k}-s\right)^{j} K_{h}\left(s_{k}-s\right)\right)=\frac{1}{h} \int(t-s)^{j} K\left(\frac{t-s}{h}\right) f(t) d t \\
& =\frac{1}{h} \int(h u)^{j} K(u) f(s+h u) d h u \\
& =h^{j} \int u^{j} K(u)\left[f(s)+h u f^{\prime}(s)+o(h)\right] d u \\
& =h^{j} f(s) \mu_{K j}+h^{j+1} f^{\prime}(s) \mu_{K(j+1)}+o\left(h^{j+1}\right) .
\end{aligned}
$$

Random variables such as $\left\{\left(s_{k}-s\right)^{j} K_{h}\left(s_{k}-s\right)\right\}_{k=1}^{n}$ actually form a independent triangular array, because the row distribution varies with the bandwidth $h$, which depends on $n$ such that $h \rightarrow 0$ as $n \rightarrow \infty$. So, by verifying the Liapunov's condition for the array such as 
$\left\{\left(s_{k}-s\right)^{j} K_{h}\left(s_{k}-s\right)\right\}_{k=1}^{n}$, one can establish the central limit theorem for the row sum. Based on the central limit theorem, there is the following approximation, which is also typically used in deriving the asymptotic variance.

$\sum_{k=1}^{n}\left(s_{k}-s\right)^{j} K_{h}\left(s_{k}-s\right)=E\left(\sum_{k=1}^{n}\left(s_{k}-s\right)^{j} K_{h}\left(s_{k}-s\right)\right)+O_{p}\left(\operatorname{Var}\left(\sum_{k=1}^{n}\left(s_{k}-s\right)^{j} K_{h}\left(s_{k}-s\right)\right)\right)$

However, all of these techniques cannot be applied to the data collected via SLAR, due to the stochastic dependence among them. If, for simplicity, we assume that all previous doses are free from truncation by the dosing limits, the estimated index at stage $n$ are

$$
\hat{s}_{k}^{(n)}=x_{k}+\hat{\phi}_{n} z_{k}=\hat{s}_{0(k-1)}+\left(\hat{\phi}_{n}-\hat{\phi}_{k-1}\right) z_{k}, \text { for } k=1, \cdots, n
$$

The index sequence $\left\{\hat{s}_{k}^{(n)}\right\}$ itself is a dependent triangular array such that each $\hat{s}_{k}^{(n)}$ depends on all data prior to stage $n$ through the estimates $\hat{s}_{0(k-1)}, \hat{\phi}_{n}$ and $\hat{\phi}_{k-1}$, where the distribution of each index $\left\{\hat{s}_{k}^{(n)}\right\}$ is not only different within a row but also varies with $n$ due to the presence of $\hat{\phi}_{n}$.

As mentioned before, another major difficulty is that the estimates of $s_{0}$ and $\phi$ are obtained numerically without explicit formulae. Thus, it is hard to determine which type of stochastic processes the index sequence belongs to, explicitly understand their stochastic behavior and postulate reasonable assumptions on their distributions. Without knowing these, it would be hard to determine to use which technique in the theory of stochastic processes.

In fact, so as to use the asymptotic theorems of stochastic processes, at least the rows of triangular array $\left\{\frac{\hat{s}_{k}^{(n)}-s}{h}\right.$, for $k=1, \cdots, n$, and $\left.n=1,2, \cdots\right\}$ have to be asymptotically stable in a certain sense.

Bosq [1996] introduces the theory of nonparametric statistics for stochastic processes, where the 3rd chapter presents the asymptotic theorems of kernel smoother with strictly 
stationary processes. Although the methodology cannot be applied to our problem directly, it inspires us that MSE of $\hat{g}_{n}(s)$ and $\hat{g}_{n}^{\prime}(s)$ (4.34) under SLAR should still converge at the same rates as those under the independent cases. Because, Theorem 3.1 of Bosq [1996] states that the MSE of kernel smoother for a stationary process converges at the rate of $n^{-4 / 5}$ which is the same as that of the independent case, if the bandwidth $h$ is selected at the optimal rate $O\left(n^{-1 / 5}\right)$. It is well known in the nonparametric regression theory that the local constant estimator, i.e. kernel smoother, converges at the same rate as the local linear estimator does. Therefore, it would be reasonable to conjecture that the dependence among data under SLAR would not impact the convergence rate of local linear estimator.

Furthermore, the most challenging part is to establish the uniform convergence (4.26) and (4.27) of the local linear estimator under SLAR. Under independent case, Stone [1982] the first time investigates the rate for local linear smoother, which result is utilized frequently in typical two-stage semiparametric regression estimation. As an extension, Hansen [2008] obtain the uniform rate for a stationary process with strong mixing condition. Based on the results of Hansen [2008], we conjecture the uniform convergence rate (4.26) and (4.27) of local linear estimator for the stochastic array $\hat{s}_{k}^{(n)}$ under SLAR.

The reason why we make the conjectures is mainly because of the difficulty in determining the type of stochastic process and explicitly investigating the stochastic behaviors for the doses and indexes in SLAR. It is also important future work to extend this research. 


\section{Chapter 5}

\section{Conclusions and Discussions}

This research is motivated by the need for individualized dosing methods in phase I cancer clinical trials, where the primary endpoint is a continuous pharmacokinetic (PK) response and dosage is also continuous. The rationale is that, in the presence of inter-subject PK variation, the doses adjusted for individual-specific needs should be safer and more efficacious than a constant dose that is common for all patients. The patient-specific dosing scheme aims at accounting for inter-individual variability in metabolizing a drug, and avoiding ethical issues sourced from underdosing or overdosing as much as possible. At first, under a linear dose-response model, we propose a repeated least squares procedure subject to a consistency constraint to realize the individualized dosing meanwhile ensuring desirable estimation precision. In the second, in order to improve the design's robustness against link misspecification, we propose a semiparametric link-adapted recursion design to individualize dose assignments while adapt for an unknown link function, under a more general semiparametric dose-response model.

This research the first time introduces the eigenvalue condition [Lai and Wei, 1982] into the model based phase I dose-finding designs. The eigenvalue constraint should be a general criterion for all parametric model based dose-finding designs, regardless whether they are 
individualized or not. Because, the eigenvalue constraint provides model based dose-finding designs with an intrinsic mechanism that can exclude the asymptotic singularity and ensure consistent estimation for model parameters. The eigenvalue constraint can also be potentially applied to other settings such as multiple covariates and binary toxicity response, as the stochastic linear regression theory is based on a multiple linear model, and it is extended to the generalized linear model setting by Chen et al. [1999]. Distinguishing from the "bestintent" designs which focus on the benefits of concurrent study subjects, RLSEVC balances the welfare of current subjects and future patients by controlling for convergence rate of the eigenvalue constraint. The design calibration plays a deterministic role in enabling the effect of eigenvalue constraint, and optimizing the balance between the current and future dosing costs. The proposed two-element loss criterion succeeds in selecting out the optimal design parameters, with which RLSEVC can not only significantly improve the precision of estimated dosing function, but also sacrifice little dosing costs. For enhanced practicality, the coherence principle is also introduced into the individualized phase I dose-finding designs for the first time. We suggest the use of coherence restriction in dose-finding designs, so as to further reduce dosing costs and avoid ethical concerns in trial implementation.

This research also pioneers in the exploration of semiparametric sequential phase I dosefinding designs. The proposed semiparametric link-adapted recursion provides a novel approach that can determine doses according to subjects' needs, while adapt for unknown link functions. Following the scheme of this approach, new individualized dose-finding designs can also be developed under other semiparametric models than the single-index models, as long as the model and dosing function have reasonable and clear interpretations. The proposed semiparametric design largely reduces the dependency of model based dose-finding designs on specific link functions, and thus gains larger applicability and higher robustness in practices. Comprehensive simulation studies demonstrate that, our proposed semiparametric design does not trade much performance for its improvement in the robustness against 
link misspecification. Under linear dose-response models, the performance of SLAR is very close to but reasonably worse than that of RLSEVC; under semiparametric dose-response models, SLAR can not only significantly improve the estimation precision for the dosing function, but can also reduce the dosing cost compared to RLSEVC. Based on the numerical simulations, we suggest that RLSEVC is a reliable choice for the first stage of SLAR; if there is prior evidence showing a nonlinear true dose-response relationship, then switching from RLSEVC to the second stage of SLAR close to the trial's end can achieve ideal dosing cost and estimation precision; if there is no strong evidence about the non-linearity, then switching from RLSEVC to SLAR at the trial's end is still a reliable scheme in practices.

It is worth to mention that, the PK measurements would be available only after the patients take the treatment. Hence, in order to adjust for PK variability prior to dose assignments, these PK measurements must be predicted, and a reliable PK model built based on sufficient pre-clinical and previous PK studies is necessary. For our proposed designs, the predicted clearance is obtained based on a prediction model between the irinotecan clearance and baseline characteristics [van der Bol et al., 2010]. Since the metabolism of irinotecan depends on several enzymes in human bodies, if the specific genotypes that determine function of these proteins could be identified accurately, it would be also reasonable to tailor dosage according to the corresponding biomarkers directly. In general, it is critical for individualized dosing to determine what individual information to adjust for, baseline characteristics, PK measurements, or biomarkers. However, the necessary intelligence supporting such decisions would exceed a statistician's knowledge range. Therefore, close inter-disciplinary collaboration among the doctors, pharmacologists, biologists and statisticians is indispensable for pooling intelligence together to support such decisions in the individualized dosing.

Unlike most conventional dose-finding methods, our proposed designs are based on a dosePK response model, rather than a dose-toxicity response model; and, our method emphasizes the balance between controlling the current dosing costs and ensuring model estimation pre- 
cision. Actually, due to the rich values of doses, early phase is a golden period to investigate the dose-PK response relationship during the entire developing course of an anti-cancer drug. As soon as the trial proceeds to later efficacy-evaluation phases, the doses would be most often fixed at two or three values. By establishing the Dose - PK response - PD endpoint relationships, the so-called PKPD model can offer more precise insights regarding a drug's efficacy and adverse effects. Such relationships are not only useful in assessing the drug's safety in early phases, but also extremely valuable in efficacy evaluation and dose justifications for the development of a novel anti-cancer drug. Moreover, the dose-finding designs based on a dose - PK response model has larger generalizability than those based on dosetoxicity outcome model, due to their applicability to studies on either cytotoxic or molecular targeted agents. Novel molecular targeted agents are more selective and less toxic. Thus, they could violate the fundamental assumption of toxicity-based dosing methods, which is the parallel increasing association between the therapeutic and toxic effects [Booth et al., 2008]. As a result, escalating dose until dose-limiting toxicity occurs does not necessarily lead to substantial increase in therapeutic effect, because the maximum efficacy might be achieved at dosage well below MTD. Therefore, for studies on a molecular targeted agent whose primary endpoint could be a continuous biomarker, the PK model based dose-finding designs are more suitable than the traditional toxicity based ones.

In terms of future work to extend this research, first of all, it would be important to extend the repeated least squares with eigenvalue constraint design to the scenarios where the primary endpoint is a binary toxicity response and/or the dosage is discrete levels. In concurrent practices, the binary toxicity response is still one of the major endpoints for phase I cancer studies, while the continuum of dosage is usual for infusion treatments and the discrete dosage is common for oral drugs. Under a generalized linear model setting, RLSEVC can be extended to binary response cases with selecting appropriate link functions. However, the extension from continuous dosage to the discrete cases is not very straightforward. For- 
tunately, to overcome this discrete barrier, Cheung and Elkind [2010] develops a promising method, the virtual observations, to bridge the continuous and discrete dosage scenarios for the stochastic approximation procedures. By applying the notion of virtual observations [Cheung and Elkind, 2010], the major obstacle of extending RLSEVC to account for the discrete dosage cases can be resolved, while the extension work is worth further systematic investigations.

At the second, it is worth to investigate the effects of the eigenvalue constraint on commonly used dose-finding designs for phase I cancer trials, for instance the continual reassessment method (CRM), and compare their performance with RLSEVC based on the first extension work. This study would target at demonstrating that the eigenvalue constraint should be used as a general criterion to ensure the estimation precision and dosing accuracy for all parametric model based dose-finding designs, no matter whether they are individualized or not.

In the third, it would be also crucial to extend the semiparametric link-adapted recursion to binary toxicity response and/or discrete dosage cases. Because, based on this extension, a comprehensive evaluation of the commonly used dose-finding designs' robustness to link misspecification can then be investigated with comparison to SLAR, so as to show SLAR's advantages on robustness over the commonly used designs. At present, such a comprehensive study is still missing for phase I dose-finding designs, and would be a meaningful supplement for assessing the robustness of the commonly used methods in practices.

At last, another significant extension work would be to establish the large sample properties of the semiparametric link-adapted recursion design. Although convincing finite sample performance usually is more important for phase I dose-finding designs due to the typical small sample size, the asymptotic properties can further provide conservative but different perspectives on evaluating the design's performance.

In the past decade, the personalized medicine has become a focus of biomedical researches, 
and is deemed as the future of medicine. As a core component, individualized dosing will inevitably replace the traditional "one-size-fit-all" dosing concept, because its matching to individual genetic makeup allows for higher safety and efficacy. Individualized dose-finding trial is indeed the first step on the pathway towards the realization of the personalized medicine in clinical practices and drug development. 


\section{Bibliography}

Babb, J. S. and Rogatko, A. (2001). Patient specific dosing in a cancer phase I clinical trial. Statistics in Medicine 20, 2079-2090.

Babb, J. S., Rogatko, A., and Zacks, S.(1998). Cancer Phase I Clinical Trials: Efficient Dose Escalation with Overdose Control. Statistics in Medicine 17, 1103-1120.

Bekele, B. N. and Thall, P. F. (2004). Dose finding based on multiple toxicities in a soft tissue sarcoma trial. Journal of the American Statistical Association 99, 26-35.

Bekele, B. N., Li, Y., and Ji, Y. (2010). Risk-group-specific dose finding based on an average toxicity score. Biometrics 66, 541-548.

Booth, C. M., Calvert, A. H., Gianccone, G., Lobbezoo, M. W., Seymour, L. K., Eisenhauer, E. A., on behalf of the Task Force on Methodology for the Development of Innovative Cancer Therapies (2008). Endpoints and other considerations in phase I studies of targeted anticancer therapy: Recommendations from the Task Force on Methodology for the Development of Innovative Cancer Therapies (MDIGT). European Journal of Cancer 44, 19-24.

Bosq, D. (1996). Nonparametric Statistics for Stochastic Processes: Estimation and Prediction. Springer-Verlag New York, Inc..

Chen, K., Hu, I., and Ying, Z. (1999). Strong consistency of maximum quasi-likelihood 
estimators in generalized linear models with fixed and adaptive designs. Annals of Statistics 27, 1155-1163.

Cheung, Y. K. (2002). On the use of nonparametric curves in phase I trials with low toxicity tolerance. Biometrics 58, 237-240.

Cheung, Y. K. (2005). Coherence principles in dose-finding studies. Biometrika 92, 863-873.

Cheung, Y. K. (2011). Dose Finding by the Continual Reassessment Method. Taylor \& Francis Group, LLC.

Cheung, Y. K., and Elkind, M. S. V. (2010). Stochastic approximation with virtual observations for dose-finding on discrete levels. Biometrika 97, 109-121.

Chow, Y. S. (1960). A Martingale Inequality and the Law of Large Numbers. Proceedings of the American Mathematical Society 11, 107-111.

Crowley, J. and Ankerst, D. P. (2006). Handbook of Statistics in Clinical Oncology (Second Edtion). Taylor \& Francis Group, LLC.

Day, S. (2007). Dictionary for Clinical Trials (Second edition). John Wiley \& Sons Ltd..

Fan, J. and Gijbels, I. (1996). Local Polynomial Modelling and Its Applications (First Edition). London: Chapman \& Hall.

Fox, E., Curt, G. A. and Balis, F. M. (2002). Clinical Trial Design for Targeted-Based Therapy. The Oncologist 7, 401-409.

Frees, E. W. and Ruppert, D. (1990). Estimation following a sequentially designed experiment. Journal of the American Statistical Association 85, 1123-1129.

Hansen, B. E. (2008). Uniform convergence rates for kernel estimation with dependent data. Econometric Theory 24, 726-748. 
Hardle, W., Muller, M., Sperlich, S. and Werwatz, A. (2004). Nonparametric and Semiparametric Models - An Introduction. Springer.

Ichimura, H. (1993). Semiparametric least squares (SLS) and weighted SLS estimation of single-index models. Journal of Econometrics 58, 71-120.

Ivanova, A. and Kim, S. H. (2009). Dose finding for continuous and ordinal outcomes with a monotone objective function: a unified approach. Biometrics 65, 307-315.

Ivanova, A. and Wang, K. (2006). Bivariate isotonic design for dose-finding with ordered groups. Statistics in Medicine 25, 2018-2026.

Kessler, M., Lindner, A. and Sorensen, M. (2012). Statistical Methods for Stochastic Differential Equations. CRC Press - Chapmann and Hall.

Kirby, S., Brain, P., and Jones, B. (2011). Fitting $\mathrm{E}_{\max }$ models to clinical trial dose-response data. Pharmaceutical Statistics 10, 143-149.

Lai, T. L. and Robbins, H. (1979). Adaptive design and stochastic approximation. Annals of Statistics 7, 1196-1221.

Lai, T. L. and Robbins, H. (1981). Consistency and asymptotic efficiency of slope estimates in stochastic approximation schemes. Z. Washrscheinlichkeitstheorie verw. Gebiete 56, 329-360.

Lai, T. L. and Wei, C. Z. (1982). Least squares estimates in stochastic regression models with applications to identification and control of dynamic systems. Annals of Statistics 10, 154-166.

Mammen, H. (1991). Estimating a smooth monotone regression function. Annals of Statistics 19, 724-740. 
Mathijssen, R. H. J., De Jong, F. A., Van Schaik, R. H. N., Lepper, E. R., Friberg, L. E., Rietveld, T., Bruijn, P. D., Graveland, W. J., Figg, W. D., Verweij, J., and Sparreboom, A. (2004). Prediction of irinotecan pharmacokinetics by use of cytochrome P450 3A4 phenotyping probes. Journal of the National Cancer Institute 96, 1585-1592.

Mukerjee, H. (1988). Monotone nonparametric regression. Annals of Statistics 16, 741-750.

O'Quigley, J., Hughes, M. D., Fenton, T., and Pei, L. (2010). Dynamic calibration of pharmacokinetic parameters in dose finding studies. Biostatistics 11(3), 537-545.

O'Quigley, J. and Paoletti, X. (2003). Continual reassessment method for ordered groups. Biometrics 59, 430-440.

O’Quigley, J., Shen, L. and Gamst, A. (1999). Two-sample continual reassessment method. Journal of Biopharmaceutical Statistics 9(1), 17-44.

O’Quigley, J., Pepe, M., and Fisher, L. (1990). Continual reassessment method: a practical design for phase I clinical trials in cancer. Biometrics 46, 33-48.

Piantadosi, S. (2005). Clinical Trials - A Methodologic Perspective (Second Edition). John Wiley \& Sons, Inc..

Piantadosi, S. and Liu, G. (1996). Improved designs for dose escalation studies using pharmacokinetic measurements. Statistics in Medicine 15, 1605-1618.

Ratain, M. J., Mick, R., Schilsky, R. L., and Siegler, M. (1993). Statistical and ethical issues in the design and conduct of phase I and II clinical trials of new anticancer agents. Journal of the National Cancer Institute 85, 1637-1643.

Ratain, M. J., Collyar, D., Kamen, B., Eisenhauer, E., Lawrence, T. S., Runowicz, C., Turner, S., and Wade, J. L. (1997). Critical role of phase I clinical trials in cancer treatment. Journal of Clinical Oncology 15, 853-859. 
Ratain, M. J. and Innocenti, F. (2010). Individualizing dosing of irinotecan. Clinical Cancer Research 16, 371-372.

Robbins, H. and Monro, S. (1951). A stochastic approximation method. Annals of Mathematical Statistics 22, 400-407.

Serfling, R. J. (1980). Approximation Theorems of Mathematical Statistics. John Wiley \& Sons, Inc..

Stone, C. J. (1982). Optimal global rates of convergence for nonparametric regression. Annals of Statistics 10, 1040-1053.

Thall, P. F., Nguyen, H. Q., and Estey, E. H. (2008). Patient-Specific Dose Finding Based on Bivariate Outcomes and Covariates. Biometrics 64, 1126-1136.

van der Bol, J. M ., Mathijssen, R. H. J., Creemers, G. M., Planting, A. S. Th., Loos, W. J., Wiemer, E. A.C., Friberg, L. E., Verweij, J., Sparreboom, A., and De Jong, F. A. (2010). A CYP3A4 phenotype-based dosing algorithm for individualized treatment of irinotecan. Clinical Cancer Research 16, 736-742.

Warfield, J. D. and Roy, A. (2013). A semiparametric sequential algorithm for estimation of dose-response curve. Sequential Analysis 32, 196-213.

Wei, C. Z. (1985). Asymptotic properties of least-squares estimates in stochastic regression models. Annals of Statistics 13, 1498-1508.

Weisberg, S. and Welsh, A. H. (1994). Adapting for the missing link. Annals of Statistics 22, 1674-1700.

Wu, C. F. J. (1986). Maximum likelihood recursion and stochastic approximation in sequential designs. In Van Ryzin, editor, Adaptive Statistical Procedures and Related Topics, 8, 1498-1508. Institute of Mathematical Statistics. 
Yuan, K. and Jennrich, R. I. (1998). Asymptotics of estimating equations under natural conditions. Journal of Multivariate Analysis 65, 245-260. 